Portland State University

PDXScholar

Fall 9-9-2019

\title{
Cooperating Teachers' Perceived Preparedness to Support Science Teacher Candidates for Culturally Sustaining Science Teaching
}

Keelan Purcell LoFaro

Portland State University

Follow this and additional works at: https://pdxscholar.library.pdx.edu/open_access_etds

Part of the Bilingual, Multilingual, and Multicultural Education Commons, Science and Mathematics Education Commons, and the Teacher Education and Professional Development Commons Let us know how access to this document benefits you.

\section{Recommended Citation}

LoFaro, Keelan Purcell, "Cooperating Teachers' Perceived Preparedness to Support Science Teacher Candidates for Culturally Sustaining Science Teaching" (2019). Dissertations and Theses. Paper 5331. https://doi.org/10.15760/etd.7204

This Dissertation is brought to you for free and open access. It has been accepted for inclusion in Dissertations and Theses by an authorized administrator of PDXScholar. Please contact us if we can make this document more accessible: pdxscholar@pdx.edu. 
Cooperating Teachers' Perceived Preparedness to Support Science Teacher Candidates for Culturally Sustaining Science Teaching

by

Keelan Purcell LoFaro

A dissertation submitted in partial fulfillment of the requirements for the degree of

Doctor of Education

in

Educational Leadership: Curriculum and Instruction

\author{
Dissertation Committee: \\ Micki M. Caskey, Chair \\ Anita Bright \\ Sybil Kelley \\ Gwen Shusterman
}

Portland State University

2019 


\begin{abstract}
In the United States, many communities face challenges that require science, technology, engineering, and math (STEM) solutions. Those communities most affected by these challenges often lack opportunities in school to use their funds of knowledge as they develop STEM literacies that would equip them to address these challenges. With new national science standards and increasingly diverse student demographics in classrooms across the United States, teacher educators must utilize strategies that prepare science teacher candidates, who are predominantly White, with pedagogies that can support diverse learners in expanding their STEM literacies from their funds of knowledge. The problem of practice guiding this research was that within the shifting landscape of STEM education, too few science teachers are prepared to implement the new standards in ways that are culturally sustaining for their traditionally underserved learners. The purpose of this convergent mixed methods study was to describe cooperating teachers' perceived preparedness to support science teacher candidates to use culturally sustaining pedagogies to inform practices and policies that influence STEM teacher preparation. To address the problem of practice quantitative and qualitative data were collected using a survey instrument and then analyzed through the lens of a conceptual framework developed called culturally sustaining science teaching. The findings suggest cooperating teachers feel "prepared" for the components of the culturally sustaining science teaching framework (curriculum, instruction, and relationships). No statistically significant differences were shown between the components but nuanced differences were apparent when quantitative mean score ranks and qualitative data were converged.
\end{abstract}




\section{Acknowledgements}

There are many people to thank for the guidance and encouragement they showed me as I moved along the journey to completing this dissertation. It is hard to find the words to express the depth of my gratitude for these people but the rest of the section is my attempt to express that gratitude. First, I would like to thank my cohort leader and committee chair, Micki Caskey, for all of her efforts in helping to not only keep me on track but also to push me to do my best work at all times. I am grateful for the wonderful combination she offered me of relentless support and insightful suggestions to help me grow as an emerging scholar and writer. I would also like to thank my other cohort leader and committee member, Anita Bright, for pushing me seek new perspectives on my work to deepen and stretch my thinking. Along with my gratitude to Micki and Anita, I am grateful for the other two members of my committee, Sybil Kelley and Gwen Shusterman, for the time they have put in to guiding my work with their unique perspectives, thoughtful questions, and constructive feedback. I know that my work improved over time because of the efforts of everyone on my committee. I also had the pleasure of working with many faculty members during my course work at Portland State University that each offered me their one of kind take on my problem of practice to help sharpen my thinking about that problem and how I may impact it as an emerging scholar. Thanks should also go to each of the members of my cohort who journeyed alongside me throughout this program for surrounding at the times I needed it the most with their personal and professional support. I am grateful to have had the opportunity to work with 
such knowledgeable and dedicated faculty and classmates during my time as a doctoral student and candidate at Portland State University.

In addition to the support of the faculty and my classmates at Portland State University, I am grateful for the guidance and encouragement I received from the leadership and my colleagues at George Fox University. The provost, Linda Samek, always found the time to talk with me about my doctoral work and how it fit into my role at George Fox. My department chairs, Carol Brazo and Kris Molitor, put in extra effort to help me navigate and effectively prioritize the demands of both my assistant professor and doctoral candidate work. My co-cohort leader with me for much of the time I worked on this study, Lynette Elwyn, was gracious and understanding with me in each step of this process. The colleague I have been co-researching with outside of this study, Donna Webb, was instrumental in helping me to envision this dissertation study in action and having been through the Portland State doctoral program was always available with relevant advice. There are also the many other colleagues I work with who would make the time to let me bounce ideas around with them or even to be present at my proposal defense. I am grateful to work with such caring and thoughtful colleagues.

I am also thankful for the efforts exerted by many people in the River School District to support me in this study. I am particularly grateful for the efforts of the district teacher on special assignment for science who persisted in communication with cooperating teachers in her district to help me gather data needed to complete this study. She went above and beyond and her help cannot be overestimated. I am also thankful to the cooperating teachers in the district who took the time over of their busy schedules as 
both science teachers and cooperating teachers to complete the survey. I am especially grateful to the two who made the extra effort to not only attend but engage for a full hour in the focus group interview.

Finally, I would be remiss if I did not mention my family whose loving foundation has put me on this path to pursing a doctoral degree and provided me the sustenance to persist to complete this dissertation. I am deeply grateful to my parents, Kevin and Carol Purcell, who have served as role models for me in how they dedicated their lives and careers to educating future generations while keeping their role as unconditionally loving and nurturing parents central to all they do. They extended a great amount of assistance in a multitude of ways during my time completing this dissertation. I am grateful also to my sister, Kelly Purcell, who inspires me to pursue my work from a place of passion and compassion and who has always been my fearless cheerleader. Thanks should also go to my mother-in-law, Patty Haughton, who also serves a model educator for me and who made the effort to support my work by reading my drafts and offering advice as well as finding and sharing relevant research for me to read to enhance my work. Most importantly, I am deeply grateful to my husband who showed unwavering support, despite the personal sacrifices necessary, and a profound belief in my abilities to complete this work. The list of ways he supports me not only on this endeavor but all of my endeavors is endless. I could not have done this without him. 


\section{Table of Contents}

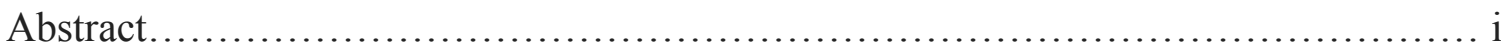

Acknowledgements........................................................... ii

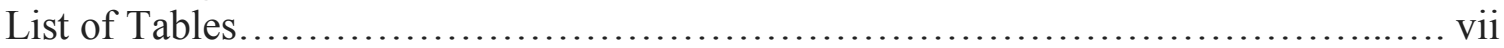

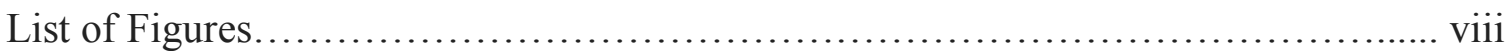

Chapter 1: Problem Statement ..................................................

Statement of the Problem .................................................. 2

Background of the Problem ............................................. 12

Significance of the Problem ................................................. 16

Presentation of Methods and Research Questions ..............................19

Definition of Key Concepts ........................................... 21

Chapter 2: Literature Review ................................................ 26

Theoretical Framework ................................................ 27

Analysis of Problem through Theoretical Framework ............................33

Conceptual Framework ................................................ 35

Critique of Theoretical and Conceptual Framework.......................... 38

Review of Research Literature .........................................4 41

Opposing Views and Practical Limitations of CSST .........................66

Review of Methods Literature ............................................69

Chapter 3: Methods......................................................... 77

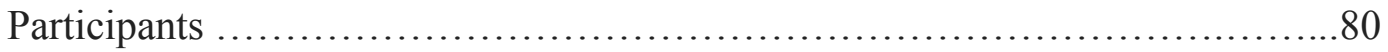

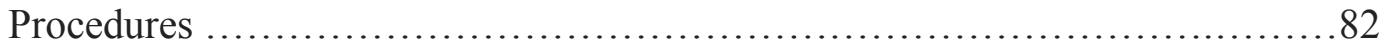

Instruments and Measures ................................................. 84

Role of Researcher .................................................... 88

Data Collection and Analysis ............................................. 89

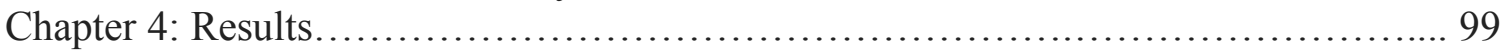

Participant Demographics ............................................ 100

Presentation and Analysis of Survey Quantitative Data ...................... 103

Interpretation of Survey Quantitative Data ................................. 119

Presentation and Analysis of Survey Qualitative Data .........................122

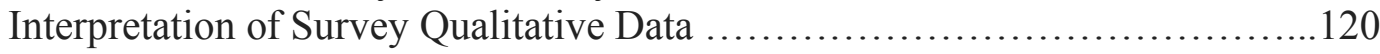

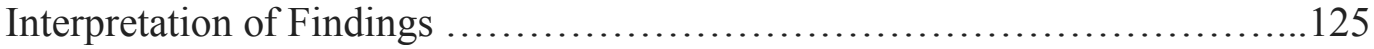

Limitations of Study ..................................................... 135

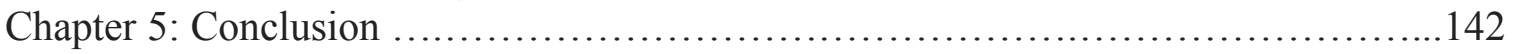

Synthesis of Findings .............................................. 144

Situated in the Larger Context......................................... 149

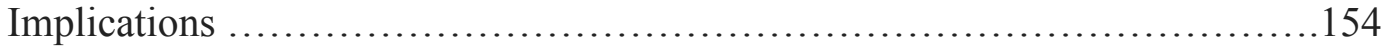

Next Steps for Research ...............................................161

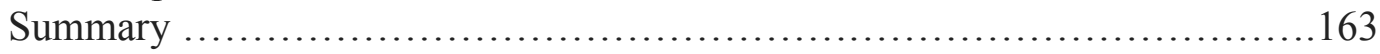

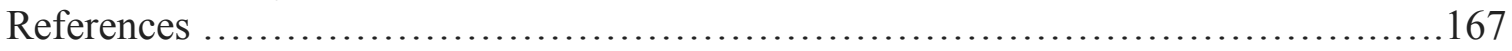

Appendix A. The Culturally Responsive Teacher Preparedness Scale ............... 181

Appendix B. Culturally Sustaining Science Teaching Preparedness Survey ............184

Appendix C. Email Script from Hsiao........................................... 193 
Appendix D. Email Script to Request Teacher Participation ............................194

Appendix E. Semi-Structure Focus Group Questions ................................196 


\section{List of Tables}

Table 1. Research Question and Data Source Alignment ...........................91

Table 2. Quantitative Survey Data Participants' Demographic Factors ................ 100

Table 3. Qualitative Survey Data Participants' Demographic Factors ...................102

Table 4. Focus Group Participants' Demographic Factors ............................... 103

Table 5. Components and Constructs of Conceptual Framework .................... 105

Table 6. Conceptual Framework Component and Construct Descriptive Statistics ......107

Table 7. Individual Survey Participant Descriptive Statistics .......................111

Table 8. Mann-Whitney Test Results Comparing Conceptual Framework Components

Table 9. Mann-Whitney Test Results Comparing Teacher and Student Demographics for Conceptual Framework Overall ......................................115

Table 10. Mann-Whitney Test Results Comparing Teacher and Student Demographics for Curriculum Component of Conceptual Framework ......................116

Table 11. Mann-Whitney Test Results Comparing Teacher and Student Demographics for Instruction Component of Conceptual Framework ....................117

Table 12. Mann-Whitney Test Results Comparing Teacher and Student Demographics for Relationships Component of Conceptual Framework ................... 118 


\section{List of Figures}

Figure 1. Conceptual model of complementary nature of culturally responsive teaching and the NGSS S\&E practices. .......................................... 8

Figure 2. Visual representation of the Culturally Sustaining Science Teaching conceptual

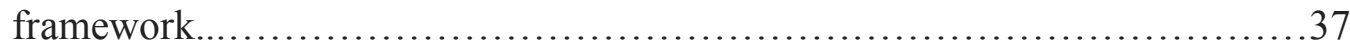




\section{Chapter 1: Problem Statement}

Our global and local communities face many challenges today that will likely affect citizens for generations to come (Farber, 2017). Many of these challenges such as the impacts of climate change or health crises require science, technology, engineering, and math (STEM) solutions. Often the populations hardest hit, especially communities of color, are simultaneously not provided opportunities in school to use their funds of knowledge (González, Moll, \& Amanti, 2005) to expand their STEM literacies. They are simultaneously disproportionately underrepresented in the STEM professions (Landivar, 2013) working to solve problems. A sense of urgency must exist not only about finding solutions to the challenges communities face, but must also focus on who is engaged in the process of working toward solutions (Landivar, 2013) to these challenges. The challenges that communities, particularly communities of color, may face in the future are dire and all members of the next generation deserve to be a part of developing solutions to the problems that will affect them and their communities. Yet, K-12 schools do not often acknowledge the ways students of color engage in STEM practices as legitimate (Civil, 2016; Delgado Bernal \& Villapando, 2016). Thus, students of color often lack opportunities when they are in school to tap into their existing scientific funds of knowledge to help them successfully navigate the border between their culture and the culture of school science (Aikenhead \& Jedege, 1999; Costa, 1995). Science teachers

and teacher educators need to be prepared to support students, particularly students of color, in successful border crossing so they can expand their science literacy skills in ways that equip them to take part in solving problems that affect their communities 
(Delgado Bernal \& Villapando, 2016; Taylor, Gillborn, \& Ladson-Billings, 2009). With more educators honoring students of color funds of knowledge, more of the next generation of students of color can be empowered to expand their science literacy skills through their funds of knowledge (Meyer \& Crawford, 2011; Rodriguez, 2015) to engage in the process of working toward solutions to problems that are likely to continue to affect them and their communities. Culturally sustaining pedagogies (Paris, 2012) that aim to "support young people in sustaining the cultural and linguistic competence of their communities while simultaneously offering access to dominant cultural competence" (p. 95) offer a promising approach to guiding educators to honor students of color funds of knowledge and support them in successful border crossing. While culturally sustaining pedagogies offer a promising approach, enacting culturally sustaining pedagogies in science classrooms requires the next generation of science teachers or science teacher candidates to be prepared with these pedagogies. The preparation of the next generation of science teachers to enact culturally sustaining pedagogies will be up to the teacher educators (i.e., university professors, university supervisors, K-12 cooperating teachers, and K-12 school administrators) that support them as they move from being science students to science teachers (Kang, Bianchini, \& Kelly, 2013).

\section{Statement of the Problem}

The landscape of STEM education in the United States has shifted in significant ways that influence the preparation of science teacher candidates to engage their students in developing science literacy (Dominguez, 2017; Windschitl \& Stroupe, 2017) through culturally sustaining pedagogies. These shifts include new national science standards 
(National Research Council, 2012) that are meant to change the means of science instruction (Januszyk, Miller, \& Lee, 2016; Krajcik, 2015) and an increasingly diverse student population in science classrooms (National Center for Education Statistics, 2017) that can benefit from culturally sustaining pedagogies (Paris, 2012; Rodriguez, 2015). The problem of practice is that within the shifting landscape of STEM education too few science teachers are prepared to implement the new standards in ways that are culturally sustaining for their traditionally underserved learners. While an established body of literature examined previous iterations of culturally sustaining pedagogies — culturally relevant pedagogy (Ladson-Billings, 1995), culturally responsive teaching (Gay, 2010), and culturally responsive, inquiry-based, science instruction (Brown, 2017; Dodo Seriki, 2018), much of the research focused on teacher candidates or current teachers, with a paucity of research on teacher educators' preparedness to guide teacher candidates to enact these pedagogies. The research conducted on teacher educators' preparedness and self-efficacy with respect to culturally relevant, responsive, or sustaining pedagogies (Thomas-Alexander \& Harper, 2017; Underwood \& Mensah, 2018; Vass, 2017) has illuminated some alarming results warranting a closer examination. I argue that a closer examination of these professionals' strengths with respect to culturally relevant, responsive, or sustaining pedagogies is critical especially within the context of STEM education as our world sees more and more challenges that require STEM solutions.

The purpose of this study was to describe the perceived preparedness of teacher educators', to support science teacher candidates seeking to teach sixth to $12^{\text {th }}$ grade students to use culturally sustaining pedagogies. Specifically, this mixed methods study 
aimed to describe the perceived strengths with respect to their preparedness of those teacher educators' who work with science teacher candidates when they are completing their clinical practice in classrooms with sixth to $12^{\text {th }}$ grade students (i.e. cooperating teachers) to inform practices and policies that impact STEM teacher preparation. This study focused on cooperating teachers' perceptions of preparedness because perceptions of preparedness influence self-efficacy which influences practice (Bandura, 2002; Matsko et al., 2018). According to Bandura (2002), "Human behavior is socially situated, richly contextualised and conditionally expressed" (p. 276). Thus, I claim that understanding cooperating teacher perceived preparedness is a crucial first step in understanding their preparedness in practice and honoring the important work these professionals do in the complex task of preparing science teacher candidates.

While the literature often distinguishes cooperating teachers from those teacher educators who work with teacher candidates in the university setting, cooperating teachers have been shown to have significant influence on the perceived preparedness of teacher candidates and their outcomes as they enter the teaching profession (Clarke, Triggs, \& Nielsen, 2014; Matsko et al., 2018; Ronfeldt, Brockman, \& Campbell, 2018; White \& Forgasz, 2016). In clinical practice models used by teacher preparation programs, the time teacher candidates spend with their cooperating teacher is often substantially greater than the time they spend with teacher educators in the university setting (White \& Forgasz, 2016). In fact, teacher candidates often cite their clinical practice experience (White \& Forgasz, 2016) and the role of their cooperating teachers (Clarke et al., 2014) as the most influential aspects of their teacher preparation programs. 
Thus, for my study, I considered cooperating teachers a crucial category of teacher educator and focused on that population of teacher educators as participants. With this mixed methods study, I aimed to inform the design and development of (a) learning experiences for cooperating teachers who host science teacher candidates, (b) strategic placements and experiences for science teacher candidates with their cooperating teachers, and (c) policies that highlight those cooperating teachers who work with science teacher candidates during the clinical practice experience.

Because I situate the problem within the new national science standards, focus on culturally sustaining pedagogies, and aim to describe teacher educators'-specifically cooperating teachers-perceived preparedness, before explaining the background of the problem and examining the problem further, I describe three critical connections that informed this study. I base these three critical connection points on a combination of theoretical and research literature. I explain in more detail the theoretical and empirical research undergirding all three of these critical connections and my associated claims for this study in Chapter 2.

First, the problem of practice guiding this study focused on culturally sustaining pedagogies that were introduced into the literature recently (Paris, 2012; Paris \& Alim, 2017) and are less broadly researched, particularly within STEM education. When describing the problem, reviewing the literature, and developing the methodology for this study, I draw heavily from the foundational research of previous iterations of culturally sustaining pedagogies_ - culturally relevant pedagogies (Ladson-Billings, 1995) and culturally responsive teaching (CResP) (Gay, 2010). In their recent text Culturally 
Sustaining Pedagogies, Paris and Alim (2017) explained the ways culturally sustaining pedagogies (CSP) build upon but are distinct from culturally relevant pedagogies (CRP), as moving forward assets-based approaches, stating:

We understand our work with CSP as founded upon the original formulation of CRP...CSP shifts toward contemporary understandings of culture as dynamic and fluid, while also allowing for the past and present to be seen merging, a continuum, or distinct, depending on how young people and their communities live race/ethnicity, language, and culture. (pp. 5-8)

The shift of CSP toward emphasizing the dynamic and fluid nature of culture and the need to not only make classrooms relevant and be responsive to cultural and linguistic diversity, but also to work to sustain the plurality of our culturally and linguistically diverse society, is a critical connection point in this study. In this study, I use Paris and Alim's (2017) shorthand CSP for culturally sustaining pedagogies as well as Underwood and Mensah's (2018) shorthand CRP for culturally relevant pedagogies and CResP for culturally responsive teaching. While these terms are often used interchangeably in the literature, I agree with Paris and Alim (2017) and Underwood and Mensah (2018) that the concepts of CRP, CResP, and CSP are distinct and not explicitly interchangeable. It is not my intention to explain all the ways these conceptual frameworks were similar or different. Rather for the purpose of this study, I acknowledged that despite these terms and concepts being distinct, I drew upon the theoretical and empirical literature related to both CRP and CResP as CSP builds upon these foundations. 
In addition to building CSP upon CRP and CResP foundations, the second critical connection point in this study was the complementarity between culturally relevant pedagogies or culturally responsive science instruction and inquiry-based science instruction promoted in the Next Generation Science Standards (NGSS) science and engineering (S\&E) practices (Brown, 2017; Dodo Seriki, 2018). In their call for the preparation of culturally responsive teachers, Villegas and Lucas (2002) drew a clear line from CResP to inquiry-based instruction grounded in constructivist approaches. In explaining how CResP is grounded in constructivism, Villegas and Lucas stated: A central task of teachers who are culturally responsive is to create a classroom environment in which all students are encouraged to make sense of new ideasthat is, to construct knowledge that helps them better understand the worldrather than merely memorizing predigested information. One way teachers can supports students' construction of knowledge is by involving them in inquiry projects that have personal meaning to them. (p. 28) Villegas and Lucas explained some specific ways they saw constructivism and inquiry being critical to culturally responsive teaching practices, many of which are complementary to those attributes for inquiry-based science instruction promoted in the NGSS such as interpreting ideas, solving problems, explaining solutions, and defending explanations. The connection between $\mathrm{CResP}$ and constructivist inquiry approaches called for by Villegas and Lucas align with the aspects of complementarity that Brown (2017) found between culturally responsive and the NGSS S\&E practices as well as those aspects of CRP that Dodo Seriki (2018) suggested to be complimentary to inquiry-based 
instruction. Because I situated the problem of practice within the context of the NGSS, I focused mainly on Brown's (2017) suggestions of complimentary which aligned CResP to aspects of the NGSS S\&E practices for the conceptual model shown in Figure 1.

The attributes of culturally responsive science instruction suggested as most complementary to attributes of the S\&E practices of the NGSS as shown in Figure 1 include: curriculum or planned learning experiences, pedagogy or instruction, and classroom relationships (Brown, 2017). For clarity, from this point forward, I refer to these attributes as curriculum, instruction, and relationships. Those attributes of the S\&E practices of the NGSS suggested as most complementary to attributes of culturally responsive science instruction, also shown in Figure 1 include connections among: (a) obtaining, evaluating, and communicating information; (b) constructing explanations and designing solutions; and (c) developing and using models (Brown, 2017).
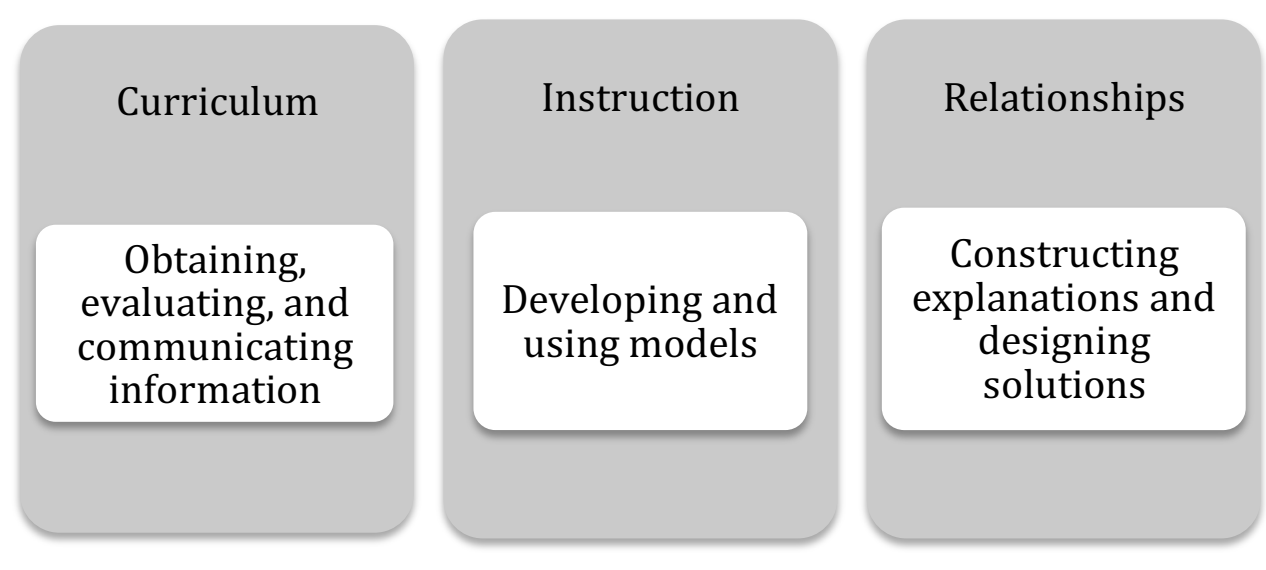

Figure 1. Conceptual model of complementary nature of culturally responsive teaching and the NGSS S\&E practices. This model builds on Brown (2017) suggestions of complementarity among the attributes of culturally responsive teaching and the NGSS S\&E practices. 
Villegas and Lucas assertions about the role of constructivist approaches such as inquiry within CResP, along with Brown's (2017) claims about the complementarity between inquiry based and culturally responsive science instruction were a critical connection point in my study. I emphasize the need to prepare science teacher candidates to use CSP not in isolation, but in the context of the shifting landscape of STEM education, which includes inquiry-based science instructional approaches outlined in the NGSS S\&E practices (National Research Council, 2011).

Finally, the third critical connection I drew in this study is the influence of cooperating teachers' readiness on teacher candidates' preparation (Anderson \& Stillman, 2013; Clarke et al., 2014; Matsko et al., 2018; Ronfeldt et al., 2018; Thomas-Alexander \& Harper, 2017; Underwood \& Mensah, 2018; Vass, 2017; Villegas \& Lucas, 2002; Windschitl \& Stroupe, 2017). Windschitl and Stroupe (2017) claimed that to move science education reform forward in ways that promote equitable instruction for traditionally underserved learners; a focus on the preparedness of teacher educators, including cooperating teachers, is necessary. Villegas and Lucas (2002) emphasized the importance in preparing culturally responsive teachers, of teacher candidates practicing culturally responsive teaching in diverse classrooms and receiving feedback from teachers who are experienced with responsive practices. In their study of cooperating teachers, Thomas-Alexander and Harper (2017) found alarming results about the deficit views and culturally responsive teaching efficacies of mentor teachers (i.e., cooperating teachers) who were supporting the development of such teacher candidates.

Additionally, in his study of barriers teacher candidates faced to culturally responsive 
teaching in their practicum experience, Vass (2017) found that the most influential barrier identified were the teacher mentors (or cooperating teachers). Even more recently and in relation to the preparation of teacher candidates for science teaching specifically, Underwood and Mensah (2018) found that university-based science teacher educators' understanding of CRP and abilities to model or provide examples of culturally relevant approaches to science was lacking, especially with respect to areas such as sociopolitical consciousness - a fundamental concept associated with CSP. While Thomas-Alexander and Harper (2017) and Vass (2017) studied mentor or cooperating teachers across a variety of subjects, Underwood and Mensah (2018) findings with university-based science teacher educators are consistent with their results highlighting how these issues are relevant to the overall preparation of science teacher candidates. Additionally, in their national study of teacher readiness for the NGSS S\&E practices, Haag and Megowan (2013) found while many teachers made positive comments about the NGSS, they indicated that "concern and in some cases outright anxiety about expectations with respect to their use of and success with the S\&E practices" (p. 423). Knowing that these same teachers could serve as teacher educators' in the role of a cooperating teacher with science teacher candidates, there appears to be a need for supporting cooperating teachers not only with respect to CSP, but also with respect to the NGSS S\&E practices. The findings from these aforementioned studies, along with the claims of Windshitl and Stroupe (2017) and Villegas and Lucas (2002), comprised the third a critical connection point in my study. Because I focused on cooperating teachers that influence science teacher candidates during their clinical practice experience, the third critical connection 
point was that the preparedness of cooperating teachers related to both the NGSS S\&E practices and CSP could influence the preparation of science teacher candidates to work with diverse learners. I argue that science teachers may be better equipped to implement the NGSS in ways that are culturally sustaining for their traditionally underserved learners if they are adequately prepared with a foundation in culturally sustaining pedagogies when they are teacher candidates. A deeply influential part of this preparation relies on the ways the teacher candidates cooperating teacher's model, promote, and provide feedback about such pedagogies.

I contend that capacity building among those teacher educators' responsible for STEM teacher preparation, particularly cooperating teachers who work with teacher candidates in the classroom as they work with students, is essential in supporting the implementation of the NGSS in ways that are culturally sustaining for traditionally underserved learners. These three critical connection points highlight the need for STEM teacher preparation research that focuses on supporting those cooperating teachers' working with science teacher candidates during their clinical experience with CSP that are complementary to the NGSS S\&E practices. Despite this need, much of the established literature on teacher preparation for CRP, CResP, or CSP in science focuses on teacher candidates or current teachers. Little research exists on cooperating teachers' preparedness to guide teacher candidates to enact these pedagogies, especially within the context of the NGSS. The research that does exist on cooperating teachers' preparedness focuses largely on what these professionals lack rather than their strengths. Thus, I argue 
for a closer examination of cooperating teachers' strengths, particularly cooperating teachers, with respect to CSP and the NGSS S\&E practices.

\section{Background of the Problem}

As mentioned previously, the landscape of STEM education has shifted in significant ways that affect the preparation of science teacher candidates. In this research, I focused on two of those shifts: (a) new national science standards, the NGSS, that emphasize inquiry-based instructional methods through specific S\&E practices; and (b) an increasingly diverse student population that can benefit from pedagogies that are culturally sustaining. The NGSS, adopted in many states across the United States starting in 2013, including Oregon (National Research Council, 2012), intended to broaden the view of what science is, how people do science, and who does science (Januszyk et al., 2016). These new standards were part of a movement in the STEM community to promote instruction that moved away from the focus on memorization of facts present in the standards of the 1990s and early 2000s.

The standards of the 1990s and early 2000s that encouraged instruction focused on memorization and regurgitation of facts grew from the accountability movement to high-stakes testing. Accountability to high-stakes testing began in the 1990s as a response to the landmark document $A$ Nation at Risk published in 1983 (Cross, 2014) and was furthered with the passage of the No Child Left Behind Act (NCLB) in 2001 when standards became coupled with high-stakes testing (Cross, 2014). NCLB affected education and science in particular, because states were more likely to adopt standards that were easier to test such as learning based on memorization of facts (Marx \& Harris, 
2006). In response to these types of standards in 2011, the policy process for a new set of national science standards, the NGSS, began. A consortium of 26 state departments of education working in collaboration with associations and organizations such as the National Research Council, National Science Teachers Association, and the Association for the Advancement of Science designed the NGSS from 2011 to 2013 (Krajcik, 2015). According to Windschitl and Stroupe (2017), research on teaching science to all learners helped guide the development of the NGSS, aimed at promoting equitable science instruction in classrooms across the United States. Yet, Rodriguez (2015) asserted that dominant cultural approaches to knowing and doing science within the scientific community heavily influenced the development of the NGSS because a "majority of the Framework Committee were Anglo males, and not science teacher educators/researchers with experience conducting research in K-12 schools" (p. 1048). Additionally, those most affected by the implementation of the NGSS in science classrooms, teacher educators, school leaders, teachers and most importantly students, were limited in their influence over the framework design (Rodriguez, 2015). Rodriguez's (2015) critiques of the NGSS highlighted the fact that the preparation of science teacher candidates to implement these standards in ways that serve their traditionally underserved learners is a complex task for teacher educators such as cooperating teachers.

The NGSS shifted “science educators' focus from simply teaching science ideas to helping students figure out phenomena and design solutions to problems" (Krajcik, 2015, p. 6). Thus, the standards also shifted the landscape of STEM education toward an inquiry-based learning approach (Bybee et al., 2006) with an emphasis on S\&E practices 
(Brown, 2017). Keeping a sense of urgency around equipping all of the next generation with science literacy skills through their funds of knowledge, makes it imperative that science teachers are prepared to provide access to inquiry-based learning approaches to science instruction, such as phenomena and design, for all students. All students should have access to instruction that moves away from memorization and regurgitation to “promote greater equity in inquiry-based science education” (Brown, 2017, p. 1146). Many scholars claim, and I agree, that despite the NGSS being based on years of research for teaching science to all students (Windshitl \& Stroupe, 2017), preparing science teachers for the standards alone is not enough to equip them to serve their traditionally underserved learners in increasingly diverse student populations (Brown, 2017; Civil, 2016; Meyer \& Crawford, 2011; Rodriguez, 2015). I suggest, as others have that science teaching that aims to serve traditionally underserved learners requires an intentional focus on sustaining the cultures of students in science classrooms across the United States, leading to the next shift in the landscape of STEM education that affects the preparation of science teacher candidates relative to my problem of practice.

In addition to the shifting focus of STEM education over the past decade toward more inquiry-based approaches, classroom student demographics have changed and will continue to change in significant ways in the United States for the next decade - making attention to equity in the implementation of the NGSS through inquiry-based science instruction vital. The National Center for Education Statistics (NCES) (2017) projected that by 2026, the proportion of White students in public schools will decrease, to account for $45 \%$ of total enrollment, while the enrollment of Hispanic students, Asian/Pacific 
Islander students, and Black students will increase, to account for more than $50 \%$ of total enrollment. CRP and CResP can be important guides for science educators hoping to advance more equitable inquiry-based science instruction (Dodo Seriki, 2018), within the framework of the NGSS S\&E practices (National Research Council, 2012) because there is suggested complementarity between these approaches (Brown, 2017) and the benefits of both are well documented in the literature. The documented benefits of inquiry-based and culturally relevant or responsive instruction include:

1. Increasing the achievement of students of color in science, as measured on assessments (Geier et al, 2008; Harris et al., 2015)

2. Guiding STEM teachers to use student-centered strategies that challenge the traditional power dynamics of the classroom (Johnson, 2011; Marshall, Smart, \& Horton, 2011)

3. Fostering more positive student-teacher relationships (Dole, Bloom, \& Kowalske, 2015; Tan \& Barton, 2010).

Unfortunately, despite the benefits and complementarity of inquiry-based and culturally relevant or responsive science instruction, the literature and teacher preparation programs often silo these concepts. Members of the education community in the United States - such as teacher educators, school administrators, and teachers themselvesclaimed that many teachers are not prepared to work with traditionally underserved students in these ways (Hawkins, 2016; Johnson, 2011; Marshall \& Smart, 2013; Moseley, Bilica, Wandless, \& Gdovin, 2014). Additionally, despite the diversification of classrooms, the teaching and teacher educator workforce has remained predominantly 
White (Dominguez, 2017; Sleeter, 2017). Taylor, Gillborn, and Ladson-Billings (2009) emphasized the detrimental impact of the lack of teacher preparedness, stating, "We are hobbled by the paradox of a largely White teaching staff whose practices, consciously or not, contribute to the racial achievement gap yet who are unable to see what they are doing" (p. 9). The lack of representation of students of color in STEM fields has made this paradox especially true for science teacher candidates preparing to become the next generation of science teachers.

\section{Significance of the Problem}

Despite all people beginning their lives with scientific capabilities (Bransford, Brown, \& Cocking, 2000), educators may not consider the funds of knowledge of some students, particularly students of color, as legitimate in K-12 schooling (Moje et al., 2004). With their funds of knowledge often invalidated, students of color are undeserved throughout their schooling and thus lack opportunities to navigate successfully the border between their home culture and the culture of school science to expand their overall science literacy skills (Civil, 2016; Delgado Bernal \& Villapando, 2016). According to Singh, Granville, and Dika (2002), many students of color become disengaged from science in their K-12 schooling years during the transitions from elementary to middle school and middle to high school, with the biggest loss of engagement being from eighth to ninth grade. The disengagement that results from being underserved magnifies the challenges students then face when working to negotiate the border between their home culture and the culture of school science to expand their science literacy skills (Britner \& Parajes, 2006), particularly during those transitional times from elementary to middle and 
middle to high school that are already full of challenges for students. Britner and Parajes (2006) asserted, "Students who do not believe that they can succeed in science-related activities...will be more likely to give up and to experience the stresses and anxieties that help ensure the erosion of their efforts" (p. 486). The disengagement of students of color from science over time resulting from lack of access to opportunities leads to a perception that students of color cannot excel in science.

When schools do not provide students of color with opportunities to expand their science literacy skills through their funds of knowledge, they reduce opportunities for students of color to participate in developing solutions to significant problems that can affect them and their communities; they rob students of color of opportunities to access fast growing and high paying careers. The challenges local and global communities face now and, in the future, that impact communities of color at higher rates than other communities make the lack of opportunity in schooling for students of color to expand their science literacy skills through their funds of knowledge, amplify the already drastic negative results of these challenges for communities of color. Along with having fewer chances to use their funds of knowledge to expand their science literacy skills throughout K-12 schooling, underserved students of color often have fewer chances to participate in STEM professions. According to Bandura (2002), people seek education opportunities based on their perceived efficacy to fill occupational roles. Bandura's claim is evident in science and engineering professions with the vast underrepresentation of people of color. In 2011, less than 15 percent of the science and engineering workforce was people of color (Landivar, 2013, p. 2). Not only do students of color deserve opportunities to 
expand their science literacy skills through their funds of knowledge, but they also deserve to be equipped to access many of the fastest growing and highest paying careers in STEM fields, should they choose to enter those professions.

In my own experience as a female student pursuing a science major, my teachers and professors' perceptions of my abilities, as well as my own perceived efficacy, affected my achievement and motivation. Despite the challenges of some of my teachers having low perceptions of me as a female pursuing science, I had a number of teachers that had positive perceptions and motivated me. I also had the privileges of race and class that afforded me with opportunities to continue to pursue my goals. When I taught middle school science in New York City and Washington, DC, I saw the effect of perceptions and efficacy on the achievement and motivation of students of color. In my classroom, I had regular reminders that all of my students wanted to be successful and were capable of success in science. When I believed in them, helped them believe in themselves, and taught them in ways that honored their strengths, we succeeded together. When I failed to engage my students in science, we failed together. In contrast, in much of the education discourse that surrounded me, I received regular reminders that my students were underachieving or at risk and that was the reason they were failing. Now as a teacher educator working to prepare the next generation of science teachers and because of my experiences as a female student pursuing science and then a science teacher working with students of color, I want to advocate for the type of teacher preparation program that promotes the instruction of science from a strengths-based perspective of all students. 


\section{Presentation of Methods and Research Question}

The problem of practice that guided this research is that within the shifting landscape of STEM education, too few science teachers are prepared to implement the new standards in ways that are culturally sustaining for their traditionally underserved learners. With the problem of practice in mind, I see the capacity building of cooperating teachers who work with science teacher candidates during their clinical practice, as essential within the shifting landscape of STEM education to promote pedagogies that serve traditionally underserved learners better. Thus, the purpose of this study was to describe the perceived preparedness of teacher educators'- specifically cooperating teachers - to support science teacher candidates to use culturally sustaining pedagogies. In this research, I addressed the following research questions:

Research Question 1: How do cooperating teachers perceive their preparedness to guide science teacher candidates to use the components (i.e., curriculum, instruction, relationships) of culturally sustaining science teaching?

Research Question 1a: To what degree do cooperating teachers feel prepared to support science teacher candidates to use the components of culturally sustaining science teaching? (Quantitative)

Research Question 1b: How do cooperating teachers describe their preparedness to support science teacher candidates to use the components of culturally sustaining science teaching? (Qualitative)

I guided this study using a mixed methods design within the pragmatic paradigm. According to Morgan (2007), a mixed methods approach is a strong methodological fit 
when the research questions require "a version of abductive reasoning that moves back and forth between induction and deduction...” (p. 71). I used a convergent mixed methods approach; specifically, what Creswell and Plano-Clark (2007) termed a validating quantitative data model. In the validating quantitative data model, the purpose of the qualitative data is to provide validation of and expansion on the quantitative results (Creswell \& Plano-Clark, 2007). Thus, in this study there were parallel quantitative and qualitative phases of data collection and analysis.

I modified and piloted survey items for use with cooperating teachers around the components of culturally sustaining science teaching as defined by my conceptual framework in Chapter 2. The quantitative phase included Likert-scale items on the Culturally Sustaining Science Teaching Preparedness Survey (adapted from Hsiao, 2015) that were a modification (with permission from the scholar) of Hsiao's (2015) Culturally Responsive Teacher Preparedness Scale (see Appendices A, B, and C). I used the quantitative data from Likert-scale items on the Culturally Sustaining Science Teaching Preparedness Survey (adapted from Hsiao, 2015) to address the Research Question (1a) by examining the degrees of preparedness expressed by cooperating teachers for each component of the conceptual framework. The qualitative phase occurred parallel to the quantitative phase as one overarching open-ended prompt at the beginning of the Culturally Sustaining Science Teaching Preparedness Survey (adapted from Hsiao, 2015) and additional open-ended prompts on the survey for each component of the conceptual framework. The purpose of the qualitative open-ended responses was to describe the strengths expressed by the cooperating teachers, to address Research Question (1b). To 
strengthen my interpretation of the survey data, I conducted a preliminary analysis of the quantitative and qualitative survey data and then shared those preliminary findings with a focus group of survey participants who indicated a willingness to participate in a focus group when they completed the survey. For this study, the purpose of the focus group was to ask the participants to speak to the accuracy of my aggregate analysis of the survey, which fits into the convergent mixed methods model as a tool for validation and member checking (Merriam \& Tisdell, 2016) rather than an additional sequence in the methods of data collection and analysis. In using the validating quantitative data model to combine the quantitative and qualitative results (and the focus group to strengthen my interpretation of those results), I gained a deeper understanding of the perceived strengths expressed by cooperating teachers to address, Research Question 1, the overarching research question.

\section{Definition of Key Concepts}

The work of various scholars, along with my own experience, guided the development of the definition of key terms for my study. I consider these definitions within the context of the problem - that too few science teachers are prepared to implement the new standards in ways that are culturally sustaining for their traditionally underserved learners - as well as within the theoretical and conceptual frameworks outlined in the next chapter.

Clinical practice experience. The time during which the teacher candidate is in a school being mentored or coached by a classroom teacher as well as an educator associated with their university program (Grossman, 2010). For this study, the focus is 
on the classroom teacher hosting a science teacher candidate who I refer to as the cooperating teacher.

Cooperating teacher. According to Clarke, Triggs and Nielsen (2014)

cooperating teacher has been the most commonly used descriptor in the literature for the professionals who work with teacher candidates during their clinical practice. Clarke at al. (2014) explained that one conception of cooperating teachers' role includes these professionals as knowledgeable and actively engaged in the meaning making process of teacher candidates, making them "teacher educators in much the same way as their university counterparts are-albeit with different responsibilities and roles" (Clarke et al., 2014, p. 168).

Culture. Smith and Dearborn (2016) defined culture as "the underlying structures, information, and messages that inform our students' upbringing, makeup, sensitivities, and how they see and fit into the world. Culture includes age, gender, ethnicity, skin color, primary language, sexual orientation, religion, socioeconomic background, and ancestry” (pp. 96-97).

Culturally sustaining. According to Paris (2012), culturally sustaining discourse builds on the work of previous culturally relevant (Ladson-Billings, 1995) and culturally responsive (Gay, 2010) scholars who explained the importance of using instruction that maintains a strengths-based approach to all students. Culturally sustaining calls on educators to consider the dynamic nature of culture and to address systems of inequity in education. 
Culturally sustaining pedagogies. Pedagogies that are culturally sustaining include but are not limited to: (a) instruction that is student-centered and promotes student agency (Ladson-Billings, 1995); (b) mindsets that are grounded in positive perceptions of all learners (Gay, 2010) that fosters students' cultural identities; (c) student-teacher relationships that recognize "dignity and care are fundamental to student and teacher learning” (Paris, 2016, p. 8); and (d) practices that call attention to, question, and challenge structural and systemic inequities (Paris, 2016).

Funds of knowledge. González, Moll, and Amanti (2005) explained that students of all backgrounds have knowledge from their own experiences and identities that they bring to their education that are assets to their learning. Teachers can and should leverage these funds of knowledge to facilitate connections between the content advanced in schools through standards and students own identities and experiences (González et al., 2005).

Inquiry-based approaches. Approaches to instruction in which the teacher uses student wonderings to guide learning of concepts and content (Bybee et al., 2006). Some examples of inquiry-based approaches are guiding students to discover phenomena and design solutions to problems that they are interested and curious about in their lives (Krajick, 2015).

Next Generation Science Standards. A framework for a three-dimensional approach to science instruction of: (a) cross-cutting concepts, (b) disciplinary core ideas, and (c) science and engineering practices (National Research Council, 2012). 
Science teacher candidates. Students pursuing a science degree endorsement in any science content area, including general science (George Fox University, n.d.) for a teaching degree.

Teacher educators. According to Swennen, Jones, and Volman (2010) teacher educators are "a professional group within education with their own specific identity and their own specific professional development needs" (p. 132). For this study, the following are included in the definition of teacher educator. Those university professors, university supervisors, K-12 cooperating teachers, and K-12 school administrators who contribute to the preparation of teacher candidates as part of their teacher preparation program. For this study, the participants are cooperating teachers who host science teacher candidates during their clinical practice experience.

Teacher preparation. Preparation includes the stage between when a teacher candidate begins a certification program and when the candidate earns a certificate (or license). During this time, candidates take coursework and participate in clinical practice in a school classroom (100kin10, n.d.).

Traditionally underserved students. Delgado Bernal and Villapando (2016) described students of color as traditionally underserved in STEM because schools are not committed to supporting all students from K-12 to graduate school to develop science literacy.

In this chapter, I introduced the problem of practice and purpose of this study. I outlined the background of the problem and contended that not only was this a significant problem but that my particular research study could provide insight to help address 
aspects of the problem. Additionally, I explained three critical connection points based on theoretical and empirical literature that guided the various aspects of this study. Finally, I identified the research questions, outlined the methods used, and defined key concepts. In the next chapter, Chapter 2, I describe the theoretical framework that guided my research, review the relevant research literature, review the methodological literature, and summarize the research literature and its application to my study. 


\section{Chapter 2: Literature Review}

In this chapter, I review theoretical, research, and methods literature related to the problem of practice that within the shifting landscape of STEM education, too few science teachers are prepared to implement the new national science standards in ways that are culturally sustaining for their traditionally underserved learners. The purpose of this study was to describe teacher educators' perceived preparedness to support science teacher candidates to use culturally sustaining pedagogies. To review from Chapter 1 , in this study I used Paris and Alim's (2017) short-hand CSP for culturally sustaining pedagogies as well as Underwood and Mensah's (2018) short-hand CRP for culturally relevant pedagogies and CResP for culturally responsive teaching. As explained in Chapter 1 , because culturally sustaining pedagogies (CSP) are foundational concepts in my research but relatively recent in the literature for this review, I drew not only from CSP literature, but also from the foundations upon which these concepts are builtculturally relevant pedagogies (Ladson-Billings, 1995) (CRP) and culturally responsive teaching (Gay, 2010) (CResP). I acknowledge that CSP is not the only conceptual approach to serving traditionally underserved learners in STEM present in the literature nor is it a new idea in the literature. Many scholars for some time have taken up the call to action to study approaches to science instruction that promote practices that are more equitable. Included in these approaches are ambitious science teaching (Windschitl, Thompson, Braaten, \& Stroupe, 2012) and sociotransformative constructivism as a guide for science instruction (Rodriguez, 1998), to name just two. While not the emphasis of the literature review, I did include some of the research within the additional approaches 
to equitable science instruction, as they are relevant to my problem of practice. In situating the problem of practice within the shifting landscape of STEM education that includes the Next Generation Science Standards (NGSS), I chose the concepts of CSP because the attributes of the foundational concepts (CRP and CResP) are complementary to attributes of inquiry-based instruction outlined in the NGSS science and engineering (S\&E) practices (Brown, 2017; Dodo Seriki, 2018). Specifically, I focused on those attributes of culturally responsive science instruction that have been suggested to be the most complementary with the attributes of the S\&E practices (Brown, 2017), shown in Figure 1 in Chapter 1. Finally, in this study, I focused on the preparation of science teacher candidates who will work at the middle and high school level (grades 6-12), so literature related to elementary science (while valuable to the knowledge base for CSP in science) was only occasionally included if it was particularly relevant to my problem of practice.

\section{Theoretical Framework}

Critical race theory and social constructivism together served as the theoretical framework that I used to analyze the literature related to my problem of practice and develop my research methods for this study. The three critical connections (outlined in Chapter 1) were important for my theoretical and conceptual frameworks as well as the development of methods for my study. For this reason, I have summarized the connected ideas in the following list:

1. Culturally sustaining pedagogies build upon the foundations of culturally relevant pedagogies and culturally responsive teaching, but are not 
interchangeable concepts (Gay, 2010; Ladson-Billings, 1995; Paris \& Alim, 2017).

2. Culturally relevant or responsive and inquiry-based science instructions are complementary in some practices and attributes (Brown, 2017; Dodo Seriki, 2018; Villegas \& Lucas, 2002).

3. The preparation of teacher educators, particularly those involved in the teacher candidates clinical experience, is crucial to the preparation of teacher candidates to work with diverse learners through strengths-based perspectives (Thomas-Alexander \& Harper, 2017; Underwood \& Mensah, 2018; Vass, 2017).

Critical race theory. The concepts of CSP align closely with critical race theory (Vass, 2017), making it a fundamental aspect of the theoretical framework for this study. The works of critical race scholars such as Ladson-Billings (2009) are foundational to the concepts of CSP. Building on the work of critical race theorists in the area of law, Ladson-Billings and others such as Taylor et al. (2009), outlined critical race theory in education. Ladson-Billings (2009) and others explained critical race theory as education at a systemic level that privileges White middle-class understandings and ways of being; and marginalizes the funds of knowledge of people of color. Critical race theorists called attention to the systemic oppression that people of color experience in the social context of education and described the role of education in challenging the systems of oppression that permeate society. According to Taylor et al. (2009), when people fail to discuss the reasons behind the achievement gap, they see this as a new problem "rather than the 
expected outcome of intentional policies and practices" (p. 7). Critical race theorists along with calling attention to the intentional policies and practices that marginalize students of color in education, called for strength-based models such as CRP, CResP, or CSP, regarding children of color, as a way to counter the deficit models that have plagued schools for so long (Taylor et al., 2009).

Ladson-Billings (1995) used critical race theory in her initial work on CRP to explain how educators could and should work toward the strengths-based model that focus on the cultural resources students bring to the classroom as a way to improve academic achievement and expand student's sociopolitical consciousness. When discussing CRP as they align to critical race theory, Ladson-Billings (2009) stated:

Fortunately, new research efforts are rejecting deficit models and investigating and affirming the integrity of effective teachers of African American students. This scholarship underscores the teachers' understanding of the saliency of race in education and the society, and it underscores the need to make racism explicit so that students can recognize and struggle against this particular form of oppression. (p. 30)

Ladson-Billings explanation of the importance of strengths-based approaches in disrupting the deficit narratives that lead to stereotypes of minority groups was foundational to this studies' theoretical grounding in critical race theory. Building on the work of Ladson-Billing's (1995) three CRP principles of academic success, cultural competence, and critical consciousness; Gay (2010) applied critical race theory to outline specific practices within CResP that she believed are needed in schools to support 
strengths-based models of students of color. Culturally responsive teaching called for a focus on: positive perceptions of parents and families of color, communication of high expectations to all learners, learning within the context of culture/culturally mediated instruction, student centered instruction that has the teacher in the role of facilitator, and reshaping the curriculum to reflect the diversity of cultures in a learning community (Gay, 2010).

In 2012, Paris argued the need for another step beyond culturally responsive teaching toward CSP. He argued for a discourse that considers "...the shifting and changing practices of students and their community" (p. 94). According to Paris (2012), educators must consider their decisions within the dynamic nature of cultures. He explained that the aim of culturally sustaining pedagogies is simultaneously to sustain the cultures of students of color and provide students of color access to the dominant culture (Paris, 2012). The evolution of concepts within CSP as they align to critical race theory grounded the first critical connection point for this study: the concepts of CSP, CRP, and CResP are not interchangeable but rather CSP builds upon the foundations of CRP and CResP (Paris \& Alim, 2017). Critical race theory and the evolution of concepts within it toward CSP not only served as a critical connection point but also guided the development of the conceptual framework and design of this study.

Social constructivism theory. The concepts of inquiry-based science instruction espoused in the NGSS S\&E practices align closely with the work of social constructivist scholars including but not limited to Dewey (1933) and Vygotsky (1978) making social constructivism another fundamental aspect of my theoretical framework. According to 
Mayer (2008), "Dewey held that the children of democracies must be apprenticed into collaborative meaning-making processes; they must be allowed to appropriate and reinvent, in terms that they can understand, the practical methods and processes currently in use within their wider society" (p. 7). Vygotsky explained in his theory of social constructivism that learning happens through interactions among individuals in the context of the culture of the knowledge community.

Every function in the child's cultural development appears twice: first, on the social level and, later on, on the individual level; first, between people (interpsychological) and then inside the child (intrapsychological). This applies equally to voluntary attention, to logical memory, and to the formation of concepts. All the higher functions originate as actual relationships between individuals. (Vygotsky, 1978, p. 57)

These foundational concepts of knowledge construction have been associated with some attributes of CResP (Gay, 2002; Villegas \& Lucas, 2002) and inquiry-based models for science instruction such as the 5E model (Bybee et al., 2006) often suggested as a guide for curriculum and instruction with the NGSS today.

In the 1980s, a step-by-step process for inquiry eventually known as the 5Es was adopted and adapted within science organizations such as Biological Science Curriculum Study. The 5Es included the following specific steps; engage, explore, explain, elaborate, evaluate (Bybee et al., 2006). The steps in the inquiry process of the 5Es align with the theory of social constructivism and CResP because these concern students' collaborative learning experiences and membership in a knowledge community-in 
which they build understanding based on their own interests and experiences (Villegas \& Lucas, 2002). In 2009, Marshall, Horton, and Smart presented a revised version of the 5Es to be the $4 \mathrm{E} X 2$. The $4 \mathrm{E} \times 2$ model added even more alignment between this inquiry process, social constructivism theory, and attributes of CResP by adding metacognition through reflection and formative assessment feedback as ways to support students in mediating the gaps between their cultural context and the context of learning in schools (Marshall, Horton, \& Smart, 2009). The development of attributes of inquirybased instruction and CResP through the lens of social constructivism theory highlights the second critical connection point I made in this study that culturally relevant or responsive and inquiry-based science instruction are complementary in some practices and attributes (Brown, 2017; Dodo Seriki, 2018; Villegas \& Lucas, 2002). Along with critical race theory, social constructivism theory also guided the development of the conceptual framework and design of my study.

Critical race and social constructivism theories together relate to the third critical connection point about the preparedness of teacher educators' (as essential parts of the knowledge community involved in teacher preparation) as crucial to the preparation of teacher candidates to work with diverse learners through strengths-based perspectives (Thomas-Alexander \& Harper, 2017; Underwood \& Mensah, 2018; Vass, 2017). In particular, teacher educators who work with science teacher candidates in the context of the clinical practice experience are interacting with the teacher candidates as they are interacting within a larger and more authentic knowledge community that is even more subject to systems that privilege White mainstream cultural understandings and practices 
than they were during their coursework. When the science teacher candidates are in their clinical practice experience, they are interacting with not only their university course instructors as teacher educators but also the cooperating teachers and university supervisors - each of whom may privilege or marginalize certain practices. Along with the interactions with these teacher educators in this broader knowledge community are interactions the science teacher candidates have with students, parents, school administrators, department, and team members that can affect their preparation for CSP. Together critical race theory and social constructivism provided a solid theoretical framework to understand the problem of practice that within the shifting landscape of STEM education, too few science teachers are prepared to implement the new standards in ways that are culturally sustaining for their traditionally underserved learners.

\section{Analysis of Problem through Theoretical Framework}

Applying critical race theory and social constructivism theory to the problem of practice reveals that science teacher candidates construct their understanding of "quality" science instruction, largely in the social cultural context of the clinical practice experience (Anderson \& Stillman, 2013). In the social cultural context of the clinical practice experience, White mainstream scientific understandings and practices are often privileged and the scientific understandings and practices of students of color are often delegitimized (Civil, 2016; Dominguez, 2017; Meyer \& Crawford, 2011; Rodriguez, 2015). Additionally, in the social cultural context of the clinical practice experience, science teacher candidates sense of preparedness is interacting with the preparedness of the various teacher educators' who work with them during this time, especially their 
cooperating teachers who they spend most of their time with while working with students. Preparing science teacher candidates for success during their clinical practice experience within the NGSS Framework of inquiry-based S\&E practices (National Research Council, 2012) alone will not necessarily advance pedagogies that serve traditionally underserved learners better (Braaten \& Sheth, 2017; Brown, 2017; Meyer \& Crawford, 2011; Rodriguez, 2015). An intentional focus on preparing science teacher candidates to use CSP (Paris \& Alim, 2017) can combat deficit narratives (Paris, 2016; Puzio et al., 2017) that marginalize certain populations in STEM (Dominguez, 2017). Most important to my study, preparing science teacher candidates to use CSP requires teacher educators' who are interacting with the science teacher candidates in the social cultural context of the clinical practice experience to feel prepared with such pedagogies.

Critical race theory and social constructivism theory illuminate some of the challenges that teacher educators', such as cooperating teachers, face when preparing science teacher candidates with pedagogies they have little opportunity to develop their readiness around (Braaten \& Sheth, 2017; Thomas-Alexander \& Harper, 2017;

Underwood \& Mensah, 2018) within a standards framework that provides little guidance for engaging traditionally underserved learners (Rodriguez, 2015). For example, a number of scholars claimed that while the connection between the preparation of teacher candidates and the preparedness of teacher educators may be evident (Bryan \& Atwater, 2002; Ferber \& Nillas, 2010; Kissau, Hart, \& Algozzine, 2017), the expectations for teacher education and teacher educators are not clear (Goodwin et al., 2014). In addition to the expectations not being clear, the expectations that do exist for teacher education 
and teacher educators tend to privilege White mainstream culture pedagogies (Sleeter, 2017) rather than those that may sustain a range of cultures including those outside the mainstream culture (Dominguez, 2017; Goodwin et al., 2014; Smith \& Dearborn, 2016). As a result of expectations for teacher preparation privileging White mainstream culture pedagogies, many teacher educators - particularly cooperating teachers - may hold deficit views of diverse learners and, rather than guiding candidates to use CRP, CResP or CSP, they can actually serve as barriers to teacher candidates using CSP (ThomasAlexander \& Harper, 2017; Vass, 2017).

Many researchers who examined the impact of teacher educators on teacher candidates (Ball, Sleep, Boerst, \& Bass, 2009; Goodwin et al., 2014; Kloser, 2014; Underwood \& Mensah, 2018; Windschitl \& Stroupe, 2017) tend to privilege the views of university professors or to focus on what teacher educators' lack (Thomas-Alexander \& Harper, 2017; Underwood \& Mensah, 2018; Vass, 2017). There is paucity of research about the strengths of those cooperating teachers that work with teacher candidates during the critically formative clinical practice experience with respect to the preparation of science teacher candidate for CRP, CResP or CSP. Thus, for my research, through the lens of critical race theory and social constructivism theory, I focused on the strengths expressed by cooperating teachers who host science teacher candidates with respect to their preparedness to support the teacher candidates to use CSP.

\section{Conceptual Framework}

In this section, I develop the claims that guided my conceptual framework through a theoretical framework of critical race theory and social constructivism theory 
with the three critical connections in mind. Using a theoretical framework of critical race and social constructivism theories applied to the problem of practice, I outlined a specific conceptual framework (i.e., culturally sustaining science teaching) based on the review of literature that guided the development of methods. Broadly, I grounded the conceptual framework in critical race theory and Gay's (2010) tenets of CResP with the addition of some of the CSP concepts put forth by Paris and Alim (2017) as building upon LadsonBillings (1995) foundational CRP work. Specifically, I based the conceptual framework on Brown's (2017) suggestions that certain attributes of culturally responsive science practices (Brown, 2017) are complementary to certain inquiry-based S\&E practices, also discussed in Chapter 1. Thus, I grounded the conceptual framework not only in the works of Ladson-Billings (1995), Gay (2010), and Paris and Alim (2017) around culturally relevant, responsive, and sustaining pedagogies through critical race theory, but also on social constructivism theory with the inquiry-based S\&E practices suggested to be most complementary to attributes of culturally responsive science (Brown, 2017) (see Figure 1). Because this complementarity was a crucial feature of my conceptual framework, I outline the details of Brown's (2017) study as well more recent similar claims (Dodo Seriki, 2018) under the review of research literature section later in this chapter. The following represents my culturally sustaining science teaching conceptual framework (Figure 2). 


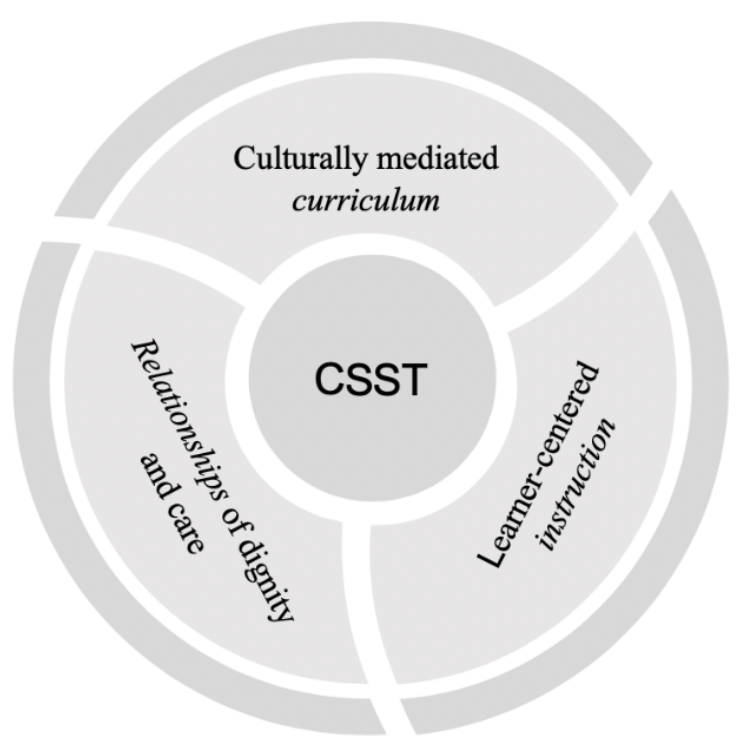

Figure 2. Culturally Sustaining Science Teaching conceptual framework. This conceptual framework is grounded in the work of Ladson-Billings (1995), Gay (2010), and Paris and Alim (2017) around culturally relevant, responsive, and sustaining pedagogies through critical race theory and on social constructivism theory with the inquiry-based S\&E practices suggested to be most complementary to attributes of culturally responsive science (Brown, 2017).

- The science teacher candidate is prepared to develop culturally mediated curriculum that includes students' cultural identities (Gay, 2010) and real world connections to students lived experiences including students obtaining, evaluating, and communicating information (Brown, 2017) about systems of power and oppression in science (Paris \& Alim, 2017).

- The science teacher candidate is prepared to facilitate learner-centered instruction that promotes agency and input from all students (Gay, 2010) and centers on collective and dynamic community languages as assets (Paris \& Alim, 2017) to learning as students develop and use models that represent a broader understanding of science concepts (Brown, 2017). 
- The science teacher candidate is prepared to foster relationships of dignity and care (Paris \& Alim, 2017) grounded in positive perceptions that communicate high expectations to all students within a collaborative learning community (Gay, 2010) where students work together to construct explanations and designing solutions to problems or challenges (Brown, 2017).

\section{Critique of Theoretical and Conceptual Framework}

Before presenting the research literature that informs this conceptual framework, I discuss assumptions and limitations of the theoretical and conceptual framework. In this section, I outline what I am not claiming and question the assumptions of using critical race and social constructivism theories to inspect what I cannot explain about the problem of practice within my chosen theoretical and conceptual framework as well based on the limitations of my positionality as a researcher. I begin the discussion with what I am not claiming in my study.

While I am claiming that there is a need to identify current areas of strength related to teacher educators' preparedness to support science teacher candidates to use CSP, I am not claiming a number of positions. First, I am not claiming that there is or should be a set way to approach or define CSP for science teaching through my theoretical framework or any other theoretical or conceptual framework. I am not claiming that my conceptual framework is an exhaustive or prescriptive description of how CSP can or should occur in science classrooms, but rather, I focus on those concepts that I see as most relevant within the shifting landscape of STEM education. Finally, and 
most importantly to me, I am not claiming that science teachers or teacher educators are to blame for the problem of practice. It is not my intention to speak negatively about students, teachers, teacher candidates, or teacher educators. Instead, as a past science teacher and cooperating teacher working in a large urban school district, as well as a current science teacher educator, my intention is to highlight and honor the important work that teacher educators do to prepare science teacher candidates to work with all of their students.

Along with what I am not claiming, I acknowledge the limitations and question the assumptions of the theoretical and conceptual framework outlined to explore the problem of practice as well my own positionality within that framework. While together critical race and social constructivism theory can provide a more detailed picture of my problem of practice, no theoretical framework can illuminate all parts of a problem. Social constructivism theory helps explain that within the social cultural context of the clinical practice experience the preparedness of those teacher educators who work with science teacher candidates influences the science teacher candidates' preparedness to implement the new national science standards in ways that are culturally sustaining for their traditionally underserved learners. Social constructivism theory as applied to my problem of practice assumes that all agents in the social cultural context of the clinical practice experience in teacher preparation have equal voice and value in that system and thus each influence one another without any differentials of power or privilege. Not considering prevalent power dynamics that exist in the social context of education (Sleeter, 2017; Taylor et al., 2009) are particularly problematic with respect to my 
problem of practice, because many science teacher candidates lack the opportunity or preparation to be critically reflexive about how their instructional decisions are either contributing to or disrupt these power dynamics. According to Bettez, Aguilar-Valdez, Carlone, and Cooper (2011), "Critical reflexivity requires teachers to actively reflect on how students are positioned in various ways by the school system and how teachers themselves might be positioning students in ways that inhibit or enhance growth and understanding" (p. 944). Thus, as a theoretical framework, social constructivism alone misses a crucial aspect of what I hoped to describe in this study, which is the strengths of teacher educators related to the various nuances of CSP including examining systems of power and privilege (Paris \& Alim, 2017).

Critical race theory calls attention to power dynamics in the social cultural context of teacher preparation thus challenging that assumption of social constructivism theory. Through critical race theory, I can see that in the social cultural context of the clinical practice experience in teacher preparation, some voices are valued and privileged more than others are; thus, those voices tend to exert more influence over the network of agents (Marion \& Gonzalez, 2014). Despite the value of having critical race theory together with social constructivism theory, there are still parts of the problem I cannot see even through the combination of social constructivism and critical race theories due to both the limitations of these theories and the limitations of my own experiences and biases. For example, a limitation of critical race theory is that there can be a danger in using counternarratives or narratives in general (Farber \& Sherry, 2016) because "one set of narratives can make us more sympathetic to people of color; it stands to reason that a different set of 
narratives can make us less sensitive" (Litowitz, 1999, p. 521). Deficit narratives are problematic for my problem of practice in particular because many science teacher candidates and teacher educators do not have opportunities to challenge the all too common narrative that low student achievement is due to low student motivation (Sleeter, 2017; Smith-Maddox \& Solórzano, 2002; Taylor et al., 2009). Thus, while critical race and social constructivism theories as lenses can illuminate some of the challenges teacher educators face when preparing science teacher candidates, within the shifting landscape of STEM, to implement the new national standards in ways that are culturally sustaining for their traditionally underserved learners, others remain unseen through these lenses alone. Finally, as no theoretical framework can illuminate all parts of a problem, no researchers experience can help them understand all parts of a problem either. My own experience in the world as a White female growing up middle class limits my understanding of the experiences of many of the students and teachers for whom I seek to advocate for in this study. I cannot and do not claim to understand what it is like to experience STEM education as a person of color either as a student, teacher candidate, teacher, or teacher educator. I discuss these limitations as they relate to my positionality as the researcher conducting this study in more detail in Chapter 3.

\section{Review of Research Literature}

Both theoretical and empirical literature informed my culturally sustaining science teaching (CSST) conceptual framework. Having explained the theoretical literature informing the conceptual framework previously, in this section I explain the empirical studies informing the framework as well as the challenges that teacher 
educators face with respect to the constructs outlined in that framework. I also synthesize the research literature within the theoretical framework and present opposing views and practical limitations of the conceptual framework.

With Brown's (2017) claim from her metasynthesis about the complementarity between some attributes of culturally responsive and inquiry-based science instruction being a crucial element undergirding my conceptual framework, I begin by outlining the details of this study including a brief description of the study upon which Brown draws her framework for analysis (Powell, Cantrell, Malo-Juvera, \& Correll, 2016). Brown (2017) conducted a metasynthesis of 52 empirical research articles on culturally responsive science instruction published "in the period between 1994...and June, 2016" (p. 1149) to determine whether there were areas of complementarity to the inquiry-based science instructional espoused in the NGSS S\&E practices. Brown (2017) used the seven attributes of culturally responsive instruction outlined by Powell et al. (2012) in the Culturally Responsive Instruction Observation Protocol (CRIOP) and the eight practices of inquiry-based science outlined in the NGSS Framework (National Research Council, 2012) to examine the connections between culturally responsive science instruction and inquiry-based science instruction in the literature. The attributes of culturally responsive instruction included in the CRIOP model (Powell et al., 2012) are:

1. Classroom relationships

2. Family collaboration

3. Assessment

4. Curriculum/planned learning experiences 
5. Pedagogy/instruction

6. Discourse and

7. Sociopolitical consciousness

Powell, Cantrell, Malo-Juvera, and Correll (2016) were interested in the potential benefits of their CRIOP model as a guide for professional development and a measure of culturally responsive instruction in the classroom; so, they conducted a mixed methods study of 27 elementary teachers. Powell et al. (2016) found, among other things, that the professional development using the CRIOP framework increased teacher implementation of culturally responsive instruction. They explained further that while professional development with the CRIOP model did result in an overall increase in teacher use culturally responsive instruction in the classroom not all attributes in the model increased; nor were all attributes in the model present in the qualitative data. For example, in their quantitative data the largest effect sizes in changes in teacher use of the attributes of culturally responsive instruction in the CRIOP model "were found for Sociopolitical Consciousness (partial $\eta 2=.41)$, Instruction (partial $\eta 2=.37)$, Assessment (partial $\eta 2=$ $.25)$, Classroom Relationships (partial $\eta 2=.19$ ), and Curriculum (partial $\eta 2=.17)$ )" (Powell et al., 2016 p. 17). The attributes of culturally responsive instruction coded from the interview transcripts with spring participant candidates as being expressed as successes were classroom relationships (9), instruction (8), and curriculum (3) (Powell et al., 2016).

Brown (2017) used Powell et al.'s (2012) framework for attributes of culturally responsive practices and looked at them in the context of science instruction, placing 
them alongside the practices of inquiry-based S\&E practices included in the NGSS

Framework, which are:

1. Asking questions and defining problems

2. Developing and using models

3. Planning and carrying out investigations

4. Analyzing and interpreting data

5. Using mathematics and computational thinking

6. Constructing explanation and finding solutions

7. Engaging in argument from evidence

8. Obtaining, evaluating, and communicating information (National Research Council, 2012)

Much like Powell et al. (2016) findings that not all aspects of the CRIOP framework increased in implementation or were expressed as successes by the candidates, Brown's (2017) findings suggest that not all of the practices and attributes of culturally responsive science instruction were complementary to the NGSS S\&E practices with the same frequency. The three practices of inquiry-based science instruction that Brown (2017) found, in her metasynthesis, to be most complementary to attributes of culturally responsive science instruction were: “(a) obtaining, evaluating, and communicating information; (b) constructing explanations and designing solutions, and (c) developing and using models" (p. 1157). The three attributes of culturally responsive science instruction that Brown (2017) found to be most complementary to attributes of inquiry-based science instruction were "(a) curriculum or planned learning 
experiences; (b) pedagogy or instruction; (c) classroom relationships" (p. 1158). More recently, Dodo Seriki (2018) suggested similar complementarity when she outlined a conceptual framework titled culturally relevant inquiry-based science pedagogy (CRISP). In this conceptual framework, Dodo Seriki (2018) outlined what she saw as significant overlaps between the attributes of culturally relevant pedagogy (CRP) and those of inquiry-based science instruction (IBSI). Some of the overlaps highlighted in Dodo Seriki's (2018) CRISP framework complemented those in Brown's (2017) metasynthesis. Those similar connections are listed below minus one overlap from Dodo Seriki's (2018) CRISP framework that was focused on assessment, which Brown (2017) did not find to be as complementary.

A. Connection to the bigger picture (IBSI). Teachers need to know the broader context and connecting students' experience to the broader context (CRP).

B. Student-centered allowing flexibility (IBSI). Promotes flexible use of students' local and global culture (CRP).

C. Content addressed using inquiry (IBSI). Teacher knows how to teach the content to the learner (CRP).

With both of these scholars suggesting this complementarity, the culturally sustaining science teaching (CSST) conceptual framework, and review of research literature that informed that conceptual framework followed those practices and attributes suggested to be most complementary. Although I focused more directly on Brown's (2017) work in the development of the conceptual framework due to its alignment with aspects of the 
NGSS, I did call upon Dodo Seriki’s (2018) claims as a way to support the development of the concepts included in that framework.

A CSST approach to STEM education. Culturally mediated curriculum, learner-centered instruction, and relationships of dignity and care can guide teachers in supporting students of color in the complex task of navigating the border between their home culture and the culture of school science (Aikenhead \& Jedege, 1999; Costa, 1995). Using these approaches in science specifically can lead to more opportunities for students of color to engage in STEM through their funds of knowledge (González et al., 2005; Moje et al., 2004). Based on their research, Aikenhead and Jedege (1999) claimed that reconciling the cultural approaches to understanding and learning science material in school with their home cultures' scientific funds of knowledge can be challenging for students of color, but success is possible if schools make the negotiation easier. In another study, Costa (1995) used interview studies to understand what influenced the perceptions of 43 high school students with respect to school science. Documentation from the interviews, classroom observations, and student records served as the primary sources of data. Costa interviewed male and female students of various racial identities enrolled in earth science or chemistry courses. Based on her findings, Costa suggested that teachers incorporate student's multiple worlds — funds of knowledge — into school curriculum and instructional practices to guide successful cultural border negotiations.

A number of scholars have explored curricula, instruction, and relationship approaches to science teaching and learning that can guide teachers to support students in successful border navigation between their home culture and the culture of school science 
(Kanter \& Konstantopoulos, 2010; Laughter \& Adam, 2012; Luft, da Cunha, \&Allison, 1998; Tan \& Barton, 2010). I explain a few of these studies and their influence on the development of my CSST conceptual framework. Luft, da Cunha, and Allison (1998) conducted a qualitative cross-case analysis of two successful high school science teachers aimed at understanding what these teachers did to support the achievement and participant of their students of color in science. In their study, Luft et al. defined a successful science teacher as one whose students earned higher grades on average, who had higher enrollment of students of color, and whose students of color continued to other science courses. Through the analysis of data collected from interviews with the teachers and their students as well as classroom observations and documents, these scholars identified themes related to these two teachers' practices. Those themes included, among other things, respect for students and their culture, and high expectations for their students of color. While an older study, the key findings of respect for student's culture, and high expectations remain consistent in much of today's literature on culturally relevant, responsive, and sustaining pedagogies in science classrooms and was thus included as an important construct in my conceptual framework with respect to developing relationships of dignity and care.

Additionally, Tan and Barton (2010) conducted an ethnographic case study of a White male science teacher working with minority students who they identified as successfully facilitating instruction that supported students in negotiating the borders between their culture and the culture of school science. In their study of Mr. M, Tan and Barton spent significant time (three times per week) with him and his students. While in 
his classroom with Mr. T and his students, Tan and Barton collected field notes, video, and interview data. Tan and Barton found that "...Mr. M engages in anti-oppressive science teaching. As the teacher, Mr. M learns where his students were coming from, building on their own out-of-school proficiencies, and making connections between students' existent knowledge and what they need to learn in science class" (p. 52). Based on what they saw as anti-oppressive science teaching in Mr. T's classroom, Tan and Barton called for science instruction that explicitly helps students build bridges between the assets of their communities and scientific understandings. Much of the literature has echoed this call, including the literature used to develop the NGSS (Windschitl \& Stroupe, 2017). Thus, I incorporated these notions as an important construct in my conceptual framework with respect to facilitating learner-centered instruction.

Also, drawing on the notions of supporting students to navigate the border between their home culture and the culture of school science Kanter and Konstantopoulos (2010) claimed that alternative science curricula (such as a project-based curriculum) can "better leverage what students bring to their science instruction rather than focusing on what they lack" (p. 856). In an attempt to understand the way in which an alternative curriculum may better leverage what students bring to their science instruction, Kanter and Konstantopoulos conducted a quantitative study using a randomized controlled trial of sixth grade science teachers in 42 schools across a racially diverse urban district. In the randomized controlled trial, both treatment groups received professional development on the NGSS Framework and then were assigned to treatment conditions of either implementing the alternative project-based science curricula (57 teachers in 21 schools), 
or the district adopted textbook curricula (51 teachers in 21 schools). In their study, Kanter and Konstantopoulos sought to identify the impact of the alternative project-based curriculum on student achievement, student science attitudes, science teacher content and pedagogical knowledge, and science teacher use of inquiry-based practices as well as the association between these factors. There were many noteworthy findings from their study but those most relevant to my conceptual framework, were their findings related to inquiry-based practices and the connections to CRP. These scholars found a connection between an increase in teacher use of inquiry-based activities in instruction, and improved student science attitudes and plan to pursue science careers. They contended that the connections found between increased use of inquiry-based activities and improved student science attitudes and plans to pursue science careers are evidence that inquiry-based instruction if further pursued within the framework of CRP could support the development of curricula that advances more equitable science education. Scholars share Kanter and Konstantopoulos's sentiment that calls for the implementation of the NGSS through alterative types of curriculum such as project, problem, and phenomenabased approaches (Dole et al., 2015; Krajick, 2015) and was thus included as an important construct in my conceptual framework with respect to developing culturally mediated curriculum. Kanter and Konstantopoulos's study, especially when considered with Tan and Barton's study, illustrates the connectedness between the components of my conceptual framework, particularly curriculum and instruction.

Related to the work of Tan and Barton (2010), and Kanter and Konstantopoulos, Laughter and Adams (2012) sought to develop and implement a culturally relevant 
science unit into the standards-based curriculum. Laughter, a White-male teacher educator, worked with Adams, a White-female middle school science teacher candidate, to develop lessons within a standards-based unit that would focus on issues of social justice in science. Laughter and Adams (2012) used an interpretive qualitative approach to describe how students engaged with the lessons to consider bias within their own lives and that of society. Adams implemented the lessons with her students in the Title I urban middle school where she had her clinical experience. Laughter and Adams (2012) explained in their results that, "we found students willing and able to wrestle with scientific content in personally relevant ways, students who came to see science as a system of knowing with its own benefits and limitations that can be employed to different ends" (p. 1129). While the findings suggest that lessons developed and taught through the social justice lens were a powerful way for students to grapple with issues of bias in science; Laughter and Adams (2012) claimed the lessons would have been more beneficial to students had they been integrated throughout the science curricula. Laughter and Adams (2012) argued that developing and implementing culturally relevant science units and lessons in the standards-based curricula could help combat the view of science as acultural because those units and lesson could be "an important tool that might convince practicing science teachers and teacher educators to begin to understand science in new ways" (p. 1128). The results of this study have resonated throughout the literature particularly the emphasis on empowering students to examine systems of power and privilege along with the challenges teachers face in implementing these approaches in the current standards-based, accountability focused political environment (Braaten \& Sheth, 
2016; Fowler, 2013; Powell et al., 2012). In the next section, I describe some of the research that reveals those challenges as well as others, which teacher educators face when preparing science teacher candidates for a CSST approach to STEM education.

Challenges preparing science teacher candidates for CSST. Despite the needs for and benefits of culturally mediated curriculum, learner-centered instruction, and relationships of dignity and care much of the literature emphasizes that preparing science teacher candidates to develop and implement curriculum, facilitate instruction, and establish relationships in these ways remains challenging for teacher educators within the shifting landscape of STEM education. Various scholars have researched the preparation of science teachers and science teacher candidates for CSST types of approaches to STEM education and while some successes are documented, the main results of these studies - especially when considered together-highlight that preparing science teacher candidates for CSST within the shifting landscape of STEM education is a complex task. For example, drawing on work of Costa (1995) around border crossing in science, Kang, Bianchini and Kelly (2013) explored through their qualitative case study the challenges that a cohort of eight teacher candidates faced when transitioning from being science students to science teachers who will take on the role of facilitating inquiry-based instructional approaches. These scholars called upon ethnographic approaches to explore the teacher candidates' experiences as they engaged in an inquiry-based experience during their coursework throughout the term as well planned and implemented inquirybased approaches to science in their student teaching experiences. Kang et al. described that teacher candidates were willing to implement inquiry instruction in their classrooms, 
but to cross this border; they needed to have mastery with skills from both the science student and science teacher world. Kang et al. noted, "A second implication from our study is that beginning teachers' willingness to, and ease in implementing inquiry may hinge on their capacity to assess and adapt instruction to their students' abilities and understandings" (p. 445). These findings point to the need to prepare science teacher candidates for CSP within the context of inquiry-based approaches.

Parallel to Kang et al. (2013) research, Dole, Bloom, and Kowalske (2015) contended that limited implementation of inquiry-based methods in the classroom may be because new science teachers experience challenges in transferring what they have learned about inquiry-based approaches in their preparation programs or professional development experiences into their practice. Dole et al. based their conclusions on their study of teachers who participated in a summer professional development experience related to project and problem-based learning instructional methods. The teacher participants engaged in online learning about project and problem-based learning and then a weeklong implementation of the instruction with students in a summer program. They collected and analyzed data from online interviews and surveys. Dole et al. explained that a dissonance between the theory and practice made the transition from learning how to teach science with the new approaches, to doing the science instruction difficult. Their results indicated that it was possible for teachers to use the new approaches when (among other factors) teachers saw the impact of such instruction on “their students' ability to direct their own learning and their own ability to let go" (p. 13). These scholar's claims are supported by what Lortie (1975) called the apprenticeship of 
observation, which in addition to the dissonance described by Dole et al., explained that many teachers will experience inconsistency between the way they learned science and the way they are being asked to teach it, especially with the shift to the NGSS.

Related to these studies of teacher's use of inquiry-based practices, and specific to the shifting landscape of STEM education with the NGSS, Haag and Megowan (2015) conducted a large-scale mixed methods study on teacher readiness for the NGSS. Their study focused on the motivations and perceived preparedness of middle and high school inservice science teachers for the NGSS S\&E practices specifically. Haag and Megowan surveyed in-service teachers from around the U.S., asking them to rate how ready they felt to implement the NGSS S\&E practices in their middle and high school classrooms. Along with the ratings, they included comment boxes in the survey that gave teachers an opportunity to elaborate on their indications with respect to the eight S\&E practices (Haag \& Megowan, 2015). The ratings served as the quantitative data and the elaborations in the comment boxes served as the qualitative data for this mixed method study. These researchers also found from the quantitative data that the high school teachers indicated both more motivation and more perceived preparation to implement the S\&E practices than the middle school teachers (Haag \& Megowan, 2015). They added, "Qualitative comments indicate that although most teachers are positive about the NGSS, they are anxious about inadequate training, limited instructional time, and lack of resources, all barriers to implementation" (p. 422). These results suggested that those inservice teachers likely now serving as cooperating teachers for new science teacher 
candidates might themselves not feel equipped for the inquiry-based approaches espoused in the NGSS.

Along with challenges associated with preparing science teachers for inquirybased approaches to science instruction, the literature documents challenges associated with preparing and supporting science teachers to use strengths-based approaches such as CRP or CResP, particularly the literature that is more recent. For example, Braaten and Sheth (2016) conducted an instrumental case study using an ethnographic approach where they followed one science teacher in two different settings, a formal science classroom, and an informal science program to explore this teacher's experiences trying to teach science for equity. They selected this teacher for their study because part of her teacher preparation program focused on equity including clinical experiences in urban schools; she was also pursuing a graduate degree in multicultural education and was demonstrating a commitment to inquiry-based approaches in her instruction (Braaten \& Sheth, 2016). Braaten and Sheth concluded after analyzing data from daily classroom observation and interviews with the teacher participant that she faced a number of tensions as she attempted to implement more equitable science practices. Among other things, Braaten and Sheth found that many of those tensions arose from the political context of teaching in a system of "accountability_embodied in science standards, assessments, and commonplace science assignments_-but were also linked to her conceptual tensions about what "counts" as scientific knowledge and practice" (p. 150). These findings about the tensions science teachers experience when trying to work in equity-oriented ways are relevant to my problem of practice as they illuminate the 
challenges teacher educators face in preparing science teacher candidates to use CSP in today's accountability focused political climate (Fowler, 2013). Adding to the challenges of implementing CSP in today's accountability focus political climate is a lack of robust understanding in the teacher preparation network of the value or use of culturally relevant, responsive, or sustaining pedagogies. Smith-Maddox and Solórzano (2002) contended, "Prospective teachers typically go through courses that focus only superficially on teaching diverse populations" (p. 67). They claimed that science teacher candidates (and all educators) need more opportunities to identify the resources that students of color bring to the classroom and to use those resources to guide their teaching.

Building on the work of Smith-Maddox and Solórzano (2002), Sleeter (2017) argued that while teacher preparation programs claim to address issues of culturally responsive teaching, a gap exists between teacher candidates' perceived understanding of their use of culturally responsive practices and the experiences in their classrooms. Sleeter described findings from her unpublished study in Critical Race Theory and Whiteness of Teacher Education indicating that despite a majority of the teachers saying they were familiar with culturally responsive teaching; most teachers still had a deficit perspective on their students, attributing low student achievement to student motivation rather than teaching. In this unpublished survey study, Sleeter had asked more than 1200 teachers, the majority of whom identified as White in two large urban districts where the majority of the student population were identified as students of color, questions about culturally responsive pedagogies. The results of Sleeter's study illuminate the gap that 
can persist for teachers, teacher educators, and teacher candidates between intentions and effect on their practices and their students.

Related to Sleeter's (2017) unpublished research that surveyed a range of teachers, Thomas-Alexander and Harper (2017) conducted a mixed-method study aimed at describing the perceptions that mentor or cooperating teachers specifically held related to working with diverse students in urban settings. Thomas-Alexander and Harper surveyed teachers a majority of whom identified as White, had earned a Master's degree, and had employment in an urban school district. Of those surveyed, less than $50 \%$ of the mentor or cooperating teachers had conducted their student teaching in an urban setting. Thomas-Alexander and Harper found on the survey - that included elements of culturally responsive teaching efficacy as well as response to the open-ended prompt in which they were asked to describe an urban classroom - the mentor or cooperating teachers "expressed overwhelmingly negative views of the students, school, and communities" (p. 49). They claimed that their results should be seen as particularly alarming because of the large influence that the beliefs of mentor or cooperating teachers could have on teacher candidates and the major role they play in the development of teacher candidates (Clarke et al., 2014; Matsko et al., 2018; Ronfeldt et al., 2018).

Parallel to and supportive of the findings of Thomas-Alexander and Harper (2017) were those of Vass (2017) who explored the preparation of teacher candidates for culturally responsive schooling practices. Vass conducted an interpretive qualitative study of three graduate teacher candidates (he termed them initial teacher educators) in their clinical practice or practicum experience in high schools in Australia. The graduate 
teacher candidates included a 25-year old Bangladeshi woman who lived in different countries and attended international schools during her formative years, a 41-year old career changer who was married to and had children with a Maori (Native Australian), and a 26-year old woman who grew up in a non-English speaking Italian household. Vass found, among other factors, that these teacher candidates faced many barriers in developing and implementing culturally relevant, responsive, sustaining curriculum. According to Vass (2017), the graduate teacher candidates:

...identified three barriers that impacted on their culturally sustaining efforts: mentors encouraging limited and limiting curricula, pedagogic and assessment practices; mentors communicating resistance to doing things differently or valuing cultural responsiveness; and a fearful awareness of being evaluated by their mentors. (p. 451)

The findings from Sleeter's (2017) unpublished survey research with current teachers coupled with Thomas-Alexander and Harper (2017) and Vass (2017) studies of mentor teachers, illustrate the elements of my problem of practice specific to cooperating teachers as science teacher educators. These studies show that cooperating teachers' effectiveness can have a significant impact on teacher candidate effectiveness as they enter the teaching profession (Clarke et al., 2014; Matsko et al., 2018; Ronfeldt et al., 2018). I contend the need for a deeper understanding of these professionals as teacher educators, especially with regard to the attributes of CSP in the context of science teaching. 
More recently and relevant to my problem of practice Underwood and Mensah's (2018) study that examined science teacher educators' (i.e., STEs) perceptions with respect to the preparation of K-12 teacher candidates (i.e., PSTs) for CRP. These scholars defined STEs as professors or adjunct professors who conducted coursework with teacher candidates. Using a two-part qualitative study that included a survey followed by participant interviews, Underwood and Mensah sought to describe "the understandings of 11 STEs had about preparing PSTs to engage diverse learners in the use of CRP” (p. 58). In sorting survey responses and coding the interview transcripts, they identified the main principles of CRP described by Ladson-Billings (1995): STEs were lacking in both their understanding of CRP and their ability to model CRP in the context of science instruction.

The STEs in this study were able to articulate the need for ideological change in science education to empower all students but were unable to describe how CRP or other substantial pedagogical changes could be used to address the needs of historically underserved or marginalized students in science classrooms. (Underwood \& Mensah, 2018, p. 59)

The results of Underwood and Mensah research, although examining a different category of teacher educators than the focus of my study, when considered with ThomasAlexander and Harper (2017) and Vass (2017) studies on mentor or cooperating demonstrate the complexity of preparing science teacher candidates to use CSP when implementing the NGSS in the shifting STEM education environment. As well as the need for further research on all type of science teacher educators who may face different 
challenges in their preparedness to support science teacher candidate to implement the NGSS in ways that are culturally sustaining for their traditionally underserved learners. While university professors and cooperating teachers may differ in many ways such as professors having different sub-identities such as that of a researcher (Swennen, Jones, \& Volman, 2010) whereas cooperating teachers may identify more with the practices of K-12 classroom instruction (Ronfeldt et al., 2018), there are also many similarities between university professors and cooperating teachers. According to Swennen et al. (2010), one of four main sub-identities of university-based teacher educators is that of a schoolteacher. "It is understandable that teacher educators identify with their former identity of school teachers because the knowledge and skills they have developed in their previous career are the basis of their knowledge and skills as teacher educators" (p. 138). Thus, while university professors and cooperating teachers may hold different roles and face different challenges in the preparation of science teachers because of their similar identifies as schoolteachers, it was logical for me to consider research literature related to both types of teacher educators, especially as it related to my problem of practice. Along with demonstrating the complexity specific to science teacher educator's preparedness to support science teacher candidates, the studies outlined in this section considered together also illuminate the interconnectedness of the various components and constructs outlined in my CSST conceptual framework.

\section{Promising approaches to prepare science teacher candidates for CSST.}

Many scholars continue to respond to the call to produce knowledge that could help guide teacher educators to overcome the aforementioned challenges. While there is a wide 
range of research available, the areas that I found particularly compelling and relevant to my problem of practice are the recent work around connecting the 5E inquiry-based model with culturally responsive practices and the work exploring teacher candidate's development of cultural humility. In the rest of this section, I outline these particular studies and the reasons I found them compelling and relevant.

First, I explain the work of Brown and Crippen (2016a, 2016b) who have taken an intentional focus on exploring those intersections of inquiry-based and culturally responsive science practices that can support the preparation of science teachers and teacher candidates. Their work is particularly relevant to my problem of practice because they include the 5E model — that the NGSS committee has recommended to guide curriculum and instruction — as a framework informing their views of culturally responsive science instruction. I found these scholars work to be promising because I think the 4E $\times 2$ (the newer iteration of the 5E model) is promising as an effective instructional model to use when preparing science teachers and teacher candidates to implement the NGSS in ways that are culturally sustaining. Brown and Crippen (2016a) stated:

Although previous research has not connected the 5E framework with culturally responsive science and math instruction, it has been shown to improve students' scientific reasoning, their interests and attitudes toward science, and subject area mastery. Thus, the framework effectively facilitates the construction and implementation of reform-based science teaching, a cornerstone of culturally responsive science teaching. (p. 116) 
I agree with Brown and Crippen's assessment of the value of the 5E model and add that the newer iteration the 4E X 2 can be beneficial specifically for the preparation of science teacher candidates, for two main reasons based on my synthesis of the research literature. First, the 4E X 2 model includes features that teacher candidate's may know from their schooling (Lortie, 1975), such as the Explain phase, which can alleviate some of the cognitive dissonance that may occur (Dole et al., 2015) making science teacher candidate's transition from science student to science teacher (Kang et al., 2013) smoother. Second, the ongoing reflection part of the model when executed with both teachers and students through a critically reflexive lens (Bettez, Aguilar-Valdez, Carlone \& Cooper, 2011; Tan \& Barton, 2010) as part of a standards-based curriculum (Braaten \& Sheth, 2016; Laughter \& Adams, 2012) can support students in navigating between their home culture and the culture of school science (Aikenhead \& Jedege, 1999; Costa, 1995).

Supporting both students and science teacher candidates to have smoother transitions is likely to increase their success with these methods in practice. Connecting inquiry-based and culturally responsive instructional approaches, Brown and Crippen (2016a) studied the impacts of a professional development entitled Science Teachers are Responsive to Students (STARTS) on early career high school life science teachers' understanding and enactment of culturally responsive science teaching. Brown and Crippen conducted a case study in which five science teachers working in high schools in one school district with a culturally and linguistically diverse student population represented a single case for the study. The teachers, all of whom were female, were of 
various ethnic backgrounds, ages, and had different years of teaching experience (Brown \& Crippen, 2016a). In their study, Brown and Crippen collected and analyzed data from interviews with the teachers, video recording of the PD experiences, and artifacts from the PD program such as units the teachers developed. Findings from the data suggested that:

For participants, adopting learner-centeredness also required some way to translate this stance to practice...Although, the act of suggesting and executing instruction that is student-centered and responsive to information learned is not in and of itself culturally responsive, for Kristen and the other teachers this action was a pathway to making instruction responsive and relevant. It was a necessary mediating process in the shift toward adopting culturally relevant practices. (p. 480)

I consider the findings that learner-centered instruction can be a pathway for teachers to use more CRP or CResP, in line with the critical connection points I outlined in my study. The findings illustrate how current teachers - those who have the potential to serve as teacher educators in the cooperating teacher role—can use learner-centered approaches to make the shift toward adopting CSP and can be better equipped to support teacher candidates to do the same.

Along with their study on the STARTS program, Brown and Crippen (2016b) developed and examined through a multiple case study the role of a structured observation protocol, called the Growing Awareness Inventory (GAIn), in building secondary science and mathematics teacher candidates' culturally responsive teaching 
capacities. Brown and Crippen conducted their study when the teacher candidates were in their field or clinical practice experience as well as taking a course on campus that emphasized equity issues. One author was the field experience instructor and the other on campus course instructor. Brown and Crippen split the 19 teacher candidates into two groups based on the endorsement areas they were pursuing (math or science); the two groups were the case of the study. They collected and analyzed data from the participant's observation responses and their lesson plans to determine whether the observation protocol affected the teacher candidates' awareness and use of culturally responsive practices. Brown and Crippen found that teacher candidates' use of the protocol during their observations of cooperating teachers delivering instruction scaffolded their awareness of culturally responsive science practices, such as: (a) facilitating a respectful and collaborative classroom environment; (b) fostering academic communication through discourse; and (c) contextualizing instruction in students' interests and experience. Unfortunately, they also found that the awareness did not always translate consistently to the teacher candidates' culturally responsive lesson plans (Brown \& Crippen, 2016b). This challenge in translating a CResP awareness to planning are consistent with Dole et al. (2015) claims that new teachers often have difficulties applying what they learn to their practice, particularly when the gap between that practice and the way they learned as students is wide (Lortie, 1975). The findings of both Brown and Crippen's (2016a, 2016b) studies are in line with the critical connection points I made in my study. The results of these studies considered together suggest that with development on, repeated exposure to, and practice with the desired attributes of inquiry- 
based S\&E practices and culturally responsive practices, teacher candidates may be better prepared to translate those practices into action. Additionally, when taken together, Brown and Crippen's (2016a, 2016b) studies further illustrate the deep interconnectedness of the components and constructs of my CSST conceptual framework, particularly with respect to curriculum and instruction.

While I claim that the work of Brown and Crippen (2016a, 2016b) are promising as a guide for preparing science teacher candidates with a solid foundation in culturally sustaining science curriculum and instruction; science teacher candidates may fall short of the desired results if they do not also hold or develop culturally sustaining pedagogical beliefs about students of color. According to Bryan and Atwater (2002), teacher candidate beliefs can influence the instructional decisions they make that, in turn, may affect the engagement of their students. Similarly, student beliefs may influence their participation in science (Britner \& Parajes, 2006), which may affect teacher beliefs and instructional decisions. Together, the connection between beliefs and curricular and instructional decisions may contribute to either furthering or disrupting deficit narratives about students of color abilities in science. Thus, preparing science teacher candidates for culturally sustaining curriculum and instruction is not enough. Teacher educators must also guide science teacher candidates to identify and combat deficit narratives about students of color's abilities in science (Delgado-Bernal and Villapando, 2016), and build relationships of dignity and care (Paris \& Alim, 2017).

Dominguez (2017) asserted that CSP that combat deficit narratives "requires affective change, a shift in ontology, in how teachers [and teacher educators] see and 
value the diversity of experiences, ways of being, and realities that exist in the world" (p. 228). Some scholars have advocated an approach to this affective change in which there is an intentional focus on cultural humility (Foronda, Baptiste, Reinholdt, \& Ousman, 2016; Hook, Davis, Owen, Worthington, \& Utsey, 2013) within teacher education. An emerging and compelling body of literature explored cultural humility in the context of teacher preparation (Lund \& Lee, 2015; Tinkler \& Tinkler, 2016). Lund and Lee (2015) and Tinkler and Tinkler (2016) studied approaches to this affective change in which there is an intentional focus on cultural humility within teacher preparation. While this teacher preparation cultural humility research is not specific to science, I included it because I claim that it could be essential to the preparation of science teacher candidates to use pedagogies that are culturally sustaining for their traditionally underserved learners. When teacher candidates and teacher educators take a cultural humility stance, the voices and views of their students, rather than societal discourse and narratives that advance deficit perspectives on students of color (Sleeter, 2017; Thomas-Alexander \& Harper, 2017; Vass, 2017), could guide science teacher candidates' reflection and empower students.

Lund and Lee (2015) studied the effects of a justice-based service learning experience in a community placement working with immigrant children and youth on teacher candidates' ability to work with diverse learners in Canada. In their interpretive study with 10 students who completed an initial placement interview, a post-placement interview, and an observation by a research assistant in their placement, Lund and Lee identified many interesting results related to cultural humility. Lund and Lee explained 
that the justice-based service learning could support teacher candidates to: (a) be more critically self-reflective, (b) take a strengths-based approach to immigrant families, and (c) build positive relationships with students. Tinkler and Tinkler (2016), who selfidentify as White "progressive educators who are committed to a constructivist approach to teaching and learning" (p. 195), conducted an interpretative study to consider how cultural humility as a lens might affect teacher candidates' work with diverse learners. Tinkler and Tinkler collected and triangulated data from reflection portfolios, questionnaires completed anonymously by both male and female teacher candidates majoring in secondary education taking a course on Reading in the Content Area and observation notes from a reflection session during the final class of the course. The themes Tinkler and Tinkler identified revealed that with cultural humility as a lens the teacher candidates were able to experience "a) discovery through stories, b) care through affirming strengths, and c) learning through reciprocal relationships" (p. 196) in detail. In interpreting the results, Tinkler and Tinkler (2016) claimed these themes could help teacher educators design programs that support the development of cultural humility as a way to promote more culturally responsive or sustaining instructional practices in classrooms. I claim that the models used in these programs (Lund \& Lee, 2015; Tinkler \& Tinkler, 2016) along with those strategies outlined in Brown and Crippen's (2016a, 2016b) studies could serve as guides for teacher educators working to prepare science teacher candidates to implement the new national science standards in ways that are culturally sustaining for their traditionally underserved learners.

\section{Opposing Views and Practical Limitations of CSST}


As shown in the literature, there are many potential benefits of a CSST approach but there are also many challenges to preparing science teacher candidates for such approaches. While I cited four studies I claim, based on a synthesis of the research, are promising strategies teacher educators could use as a guide when preparing science teacher candidates, there are substantial practical significances and opposing views that I discuss in the rest of this section through the theoretical framework of critical race and social constructivism theory. First, because I situate the problem of practice within the NGSS and claim, as others have (Bybee, 2014) that some practices espoused by the NGSS may be valuable in preparing science teacher candidates, it is important to consider the view mentioned in Chapter 1 that the NGSS Framework lacks a focus on engagement, equity, and diversity (Rodriguez, 2015). According to Rodriguez (2015), the NGSS fails to provide concrete examples of "how to make the science content culturally relevant and inclusive" (p. 1042) thus ultimately privileging White mainstream scientific understandings and practices. Critical race theory applied to this view, prompts science teacher candidates and teacher educators to consider what is legitimate in science education, how our evaluation methods contribute to that legitimization, and ultimately the teaching and learning that occurs with students as a result. According to DarlingHammond (2006), preparing teachers for the approaches espoused in policies requires explicit modeling of practices, especially when those practices are markedly different from a teacher's own experiences as a learner (Bransford et al., 2000; Lortie, 1975). Social constructivism theory applied to Rodriguez (2015) and Darling-Hammond (2006) views suggests that even with an intentional focus on the connections between the NGSS 
inquiry-based S\&E practices and culturally responsive practices, there is a practical limitation of little guidance in the social cultural context of teacher preparation for preparing science teacher candidates with pedagogies that may be culturally sustaining.

Second, because I situate the problem of practice around the preparation of science teacher candidates in university-based programs, it is important to point out the view that the current evaluation method the Teacher Performance Assessment (edTPA) directed nationally with teacher candidates, including science teacher candidates mobilizes bias (Fowler, 2013) toward White cultural dominance (Dover \& Schultz, 2016). According to Dover and Schultz (2016), such teacher testing tends to reinforce White dominance rather than encourage culturally mediated teacher preparation or instruction at the K-12 level. Applying critical race theory to Dover and Schultz's claim reveals that the current evaluation of science teacher candidates may risk shaping the consciousness of what the education community considers quality science teaching to bias and favor White mainstream scientific understandings and practices (Dominguez, 2017). According to Fowler (2013), the evaluation methods chosen to establish the success of a desired policy matter greatly because teachers choose different methods of instruction depending on the assessment methods that accompany the policies. Social constructivism theory applied to both Dover and Schultz (2016) and Fowler (2013) claims revealed that certain evaluation methods create practical limitations in the social cultural context of teacher preparation because they impede teacher educator's ability to promote certain methods of instruction if they do not help the candidates achieve success on the evaluation. 
I claim that the aforementioned views and practical limitations make the need for the research outlined in my study even more urgent. I contend that describing cooperating teacher's preparedness with respect to the CSST conceptual framework within the theoretical framework of critical race theory and social constructivism will be a valuable contribution to the STEM education research literature. In the next section, I outline the literature on mixed methods research that I used to guide the selection and design of methods for this study, which I explain in detail in Chapter 3.

\section{Review of Methods Literature}

As explained in Chapter 1, a convergent mixed methods approach, specifically a validating quantitative data method (Creswell \& Plano-Clark, 2007), is a proper match for the research questions I aimed to address in this study:

Research Question 1: How do cooperating teachers perceive their preparedness to guide science teacher candidates to use the components (i.e., curriculum, instruction, relationships) of culturally sustaining science teaching?

Research Question 1a: To what degree do cooperating teachers feel prepared to support science teacher candidates to use the components of culturally sustaining science teaching? (Quantitative)

Research Question 1b: How do cooperating teachers describe their preparedness to support science teacher candidates to use the components of culturally sustaining science teaching? (Qualitative)

Thus, my review of methods literature focuses on mixed methods and survey methods research with some literature on focus group interviews as well, as this approach could 
strengthen the interpretation of the survey data. I cite the work of various scholars (i.e., Boone \& Boone, 2102; Carifio \& Perla, 2008; Creswell, 2014; Creswell \& Plano-Clark, 2017; Fowler, 2009; Morgan, 1996, 2014) to inform the selection and design of my mixed methods approach for this study.

To begin, Morgan (2014) offered a guide for how the pragmatic paradigm informs mixed methods as a methodology. Then, Creswell (2014) provided an overview of mixed methods research more generally, while Creswell and Plano-Clark (2007) guided a deeper dive into mixed methods research, specifically the details of design within the various approaches. Next, Fowler's (2009) work informed my thinking about survey research. Additionally, the work of Boone and Boone (2012) and Carifio and Perla (2008) provided more specifics regarding Likert-scale specific surveying and analysis. Finally, Morgan (1996) presented useful information about focus groups as a data collection method, particularly when used in combination with other methods as in my study.

I begin with an explanation of the pragmatic paradigm that guides my selection of mixed methods as a methodology, and why it is an appropriate paradigm for me as a researcher and for my study in particular. According to Morgan (2014):

Combined with the belief that the world is both real and socially constructed, pragmatists also believe that all knowledge is social knowledge...Pragmatists thus acknowledge that each individual's knowledge is unique because it is based on individual experience, while also asserting that much of this knowledge is socially shared because it comes from socially shared experiences. (p. 39) 
Morgan's (2014) explanation of the stance of many pragmatic researchers confirmed that a pragmatic paradigm is a strong fit for my methodological framework because I use both the principles of critical race theory and social constructivism as the foundational theoretical framework for this study. Applied to my problem of practice through the lens of critical race and social constructivism theories, the pragmatic methodological view asserts the existence of reality along with the social construction of that reality. In this case, the oppression students of color experience in STEM education including how individual's knowledge of that oppression draws upon their unique experiences and how those experiences are socially constructed with others experiences. This view of knowledge and knowledge construction within the pragmatic paradigm not only fits my theoretical framework, but it also aligns with my ontological and epistemological views.

Pragmatists tend to conduct research in a methodological middle ground between realism and constructivism because they are mainly interested in why a researcher wants to do their study and the studies practical implications (Morgan, 2014). Some scholars claim that survey research needs to be reserved for realism and participatory research for constructivism but pragmatists claim that selection of methods should be more about the purpose of the research than the ontological situation of the methods (Creswell \& PlanoClark, 2007). According to Creswell (2014), "Mixed methods is chosen because of its strength of drawing on both qualitative and quantitative research and minimizing the limitations of both approaches" (p. 218). Thus, with the purpose of this study in mind, I drew upon the pragmatic paradigm as a guide for the research methodology. 
Within a mixed methods research design, a researcher could use a variety of specific methods approaches, such as convergent or triangulated methods, exploratory methods, or explanatory methods (Creswell \& Plano-Clark, 2007) depending upon the goals of the study, the details of the selected approach of the design elements would vary. For example, a convergent method, is likely to be conducted as a single-phase study where data are collected and analyzed in parallel fashion and then brought together to address the research questions (Creswell \& Plano-Clark, 2007). The emphasis in a convergent mixed methods approach can be equal between the quantitative and qualitative data or can situate one as more central than the other (Creswell \& PlanoClark, 2007). Exploratory and explanatory designs are different from convergent designs in that data collection and analysis occur in phases. For example, in an exploratory mixed methods design, the researcher collects and analyzes qualitative data to inform the collection and analysis of quantitative data (Creswell, 2014). The opposite is true of an explanatory mixed methods design, in which the researcher collects and analyzes quantitative data to inform the collection and analysis of qualitative data (Creswell, 2014). After reviewing these mixed methods research designs, I selected a convergent method as most appropriate for my study.

With regard to survey research, Fowler (2009) has written extensively on the use of surveys in research. While Fowler (2009) does not address mixed methods research designs specifically, I applied his survey design approach to the work of Morgan (2014), Creswell (2014), and Creswell and Plano-Clark (2007) related to mixed methods design. According to Fowler (2009): 
...every survey involves a number of decisions that have the potential to enhance or detract from the accuracy (or precision) of survey estimates... Thus the design of a survey involves a set of decisions to optimize the use of resources. Optimal design will take into account all the salient aspects of the survey process. (p. 7) I highlight Fowler's point as it related to the use of a convergent mixed methods survey design through the pragmatic paradigm in my study. I provide details of the optimal design for my study in Chapter 3. To optimize the design in its ability to describe teacher educators' perceived strengths with respect to their preparedness to support science teacher candidates to use pedagogies that are culturally sustaining for traditionally underserved learners within the CSST conceptual framework, I used validating quantitative methods (Creswell \& Plano-Clark, 2007). Specifically, I embedded openended prompts in the Culturally Sustaining Science Teaching Preparedness Survey (adapted from Hsiao, 2015) as a way to validate and expand the findings of the quantitative Likert-scales on that same survey.

According to Boone and Boone (2012), researchers who survey with Likert-scales need to clarify the content of their Likert-scale as well as the use and analysis of Likerttype questions. For this study, I adapted Hsiao's (2015) Culturally Responsive Teacher Preparedness Scale to use with science cooperating teachers. Hsiao's scale included a Likert-scale for each component of the conceptual framework and those scales consisted of Likert-type questions associated with individual constructs within each component. Thus, the analysis of my conceptual framework components was different from the analysis of the constructs within those components. The components in my conceptual 
framework (i.e., curriculum, instruction, relationships) appear in the Culturally

Sustaining Science Teaching Preparedness Survey (adapted from Hsiao, 2015) as a set of five individual Likert-type questions representing the constructs within that component. More basic analyses, such as descriptive statistics are appropriate (Boone \& Boone, 2012) for the individual construct Likert-type items whereas more sophisticated analyses such as parametric or non-parametric statistical tests (Carifio \& Perla, 2008) are useful for the combination of those constructs into the three major components of the conceptual framework. Carifio and Perla (2008) justified the use of more sophisticated statistical analyses for Likert-scales specifically because they argued that when used as true scales - or the combination of multiple Likert-type items (Boone \& Boone, 2012) - then the resulting scale numbers can be compared to each other as either intervals with parametric statistical testing or ordinals with non-parametric statistical testing. Thus, when outlining and conducting data analysis for this study, I relied on the arguments and explanations of Boone and Boone (2012) and Carifio and Perla (2008).

Along with embedding open-ended prompts in the survey, I employed an additional qualitative approach of focus group interviews that followed what Morgan (1996) explained as one of a variety of uses for focus groups in combination with survey and one that while less common is increasing its use in social science research. The approach I used in this validating quantitative data convergent mixed methods study "is to recontact survey respondents for illustrative material that can be quoted in conjunction with quantitative findings" (p. 135). Specifically, in this study, I used focus group interviews after an initial analysis of both the quantitative and qualitative survey data to 
strengthen my interpretation of these results. According to Morgan (1996), a number of scholars have examined the similarities and differences in use and usefulness of surveys compared to focus groups. In summarizing the ways other scholars have seen these similarities and differences, Morgan (1996) explained that these approaches always had differences in which the focus group provided depth while the survey provided breadth. In choosing to use a focus group interview method in combination with the survey method, I aimed to add to the breadth of my survey findings with depth from the focus group interview, particularly to strengthen my interpretation of the converged findings of the quantitative and qualitative survey data. Recent mixed methods survey studies were especially relevant to my study in both content and methods. Thus, along with building upon the work of Morgan (2014), Creswell (2014), Creswell and Plano-Clark (2007), Fowler (2009), and Morgan (1996), I drew upon the findings of mixed methods survey studies (Haag \& Megowan, 2015; Thomas-Alexander \& Harper, 2017). These survey studies indicated that science teachers felt unprepared for the S\&E practices outlined in the NGSS, and cooperating teachers expressed low culturally responsive teaching selfefficacies. In my study, I aimed to highlight the ways in which cooperating teachers, as teacher educators feel prepared and express strengths with respect to supporting science teacher candidates. I describe how these studies informed my methods and how I aimed to highlight the strengths of these professionals more in the next chapter.

In this chapter, I described the theoretical and conceptual framework guiding this study. I synthesized the theoretical and research literature informing the theoretical and conceptual framework as well as the design of this study. Additionally, I examined the 
methods literature related to mixed methods, survey research, and focus group interviews.

In the next chapter, Chapter 3, I describe the details of the methods for this study

including participants, procedures, instruments, and measures, role of researcher, and data collection and analysis. 


\section{Chapter 3: Methods}

An established body of literature examines the preparation of science teachers for culturally relevant pedagogies (CRP) and culturally responsive teaching (CResP) (Braaten \& Sheeth, 2017; Brown \& Crippen, 2016a; Brown \& Crippen, 2016b; Johnson, 2011; Laughter \& Adam, 2012; Thomas-Alexander \& Harper, 2017; Underwood \& Mensah, 2018). An emerging body of literature also examines teacher preparation for culturally sustaining pedagogies (CSP) (Puzio et al., 2017; Vass, 2017). Nevertheless, there is a paucity of research around preparing science teacher candidates for CSP, especially within the context of the NGSS. In my study, I aim to address two gaps in the literature. First, the literature on science teacher preparation could benefit from more research that examines CSP as built upon the concepts of CRP and CResP. Second, the literature on teacher preparation for CRP, CResP, and CSP could benefit from research that considers the perceptions of a broad range of teacher educators, as these are the professionals who influence the preparation of teacher candidates - ultimately affecting K-12 student's opportunities to expand their STEM literacies through their funds of knowledge. In this study, I contend that understanding the perceived strengths of cooperating teachers as teacher educators who prepare science teacher candidates would add to the knowledge base and contribute to the goal of working toward serving traditionally underserved learners in STEM. My research is particularly relevant and necessary in the context of recent findings about the perceptions and efficacies of cooperating (or mentor) teachers with respect to CRP and CResP (Thomas-Alexander \& Harper, 2017; Underwood \& Mensah, 2018; Vass, 2017). With my study, I intend to add 
to the literature that informs the design and development of (a) learning experiences for teacher educators, (b) more strategic clinical practice experiences for teacher candidates, and (c) policies that professionalize the role of those teacher educators that work with teacher candidates during the clinical practice experience.

The problem of practice guiding my study was that within the shifting landscape of STEM education, too few science teachers are prepared to implement the new standards in ways that are culturally sustaining for their traditionally underserved learners. The purpose of my study was to describe teacher educators'-specifically cooperating teachers-perceived preparedness to support science teacher candidates to use culturally sustaining pedagogies. The following research question addressed cooperating teachers' perceived preparedness:

Research Question 1: How do cooperating teachers perceive their preparedness to guide science teacher candidates to use the components (i.e., curriculum, instruction, relationships) of culturally sustaining science teaching?

As mentioned in Chapter 1, to address this overarching research question for my study required "a version of abductive reasoning that moves back and forth between induction and deduction..." (Morgan, 2007, p. 71). Thus, for my study, I used mixed methods as a methodology within the pragmatic paradigm (Morgan, 2007). Creswell and Plano-Clark (2007) suggested when using a mixed methods approach to have, along with the overarching research question, a set of sub-questions associated with both the quantitative and qualitative aspects of the research. Considering this suggestion, I developed two subquestions for my convergent mixed methods research: 
Research Question 1a: To what degree do cooperating teachers feel prepared to support science teacher candidates to use the components of culturally sustaining science teaching? (Quantitative)

Research Question 1b: How do cooperating teachers describe their preparedness to support science teacher candidates to use the components of culturally sustaining science teaching? (Qualitative)

According to Creswell and Plano-Clark (2007), researchers can use a variety of specific methods approaches within the mixed methods methodology to guide data collection and analysis. They contend that the selection of one method over another needs to be determined by the specific goals of the research. With the goal of this study in mind, I chose a convergent mixed methods approach (Creswell, 2014). A convergent mixed methods approach allowed me to use quantitative and qualitative data simultaneously to develop a more full description of teacher educators' perceived strengths with respect to their preparation to support science teacher candidates to use CSP to answer the overarching research question (see Table 1). Within the convergent mixed methods approach specifically, I used what Creswell and Plano-Clark (2007) termed a validating quantitative data model as part of a triangulation design. According to Creswell and Plano-Clark (2007) a validating quantitative data model is especially useful when researchers "want to validate and expand on the quantitative findings from a survey by including a few open-ended qualitative questions" (p. 65).

Within the validating quantitative data model, the procedure for data collection and analysis reflected the methods of two recent studies particularly relevant in both 
content and methods to my problem of practice and my study's purpose. Both Haag and Megowan's (2015) mixed methods study of teacher readiness for the NGSS, and Thomas-Alexander and Harper's (2017) mixed methods study of cooperating teacher culturally responsive teaching self-efficacies guided the selection of methods for my study. These researchers justified the use of convergent mixed methods as a way to add breadth and depth to their understanding of the research questions (Creswell, 2014; Morgan, 2007) they sought to address. In using a convergent mixed methods approach and mirroring the methods of these scholars, I planned to bolster the validity and reliability of my methods and address some of the concerns about the quantitative approaches to research of CRP, CResP, and CSP.

\section{Participants}

For my study, I used purposeful selection of the school district and the participants within the district rather than a random sample (Fowler, 2009). As part of the purposeful selection of the school district, I made a preliminary inquiry with the science teacher on special assignment (TOSA) in a large culturally and linguistically diverse district the Portland Metro Area, the River School District (pseudonym). I selected this district as a prospective research site and thus made preliminary inquiries with the TOSA there for a number of reasons. First, I estimated that a higher number of cooperating teachers in this district host science teacher candidate each year relative to other districts in the area. Second, I had met the science TOSA of this district who agreed to support the research efforts outlined in my study proposal, so I anticipated that with her assistance I could achieve a good response rate. Third, this district espouses an 
emphasis on promoting equity in their STEM instruction, and they remain at the forefront in the Portland Metro Area of NGSS aligned phenomena-based curriculum development. Teachers within River School District_-some of whom ended up being a part of the cooperating teacher sample—-have a reputation for their equity work.

My hope was that by selecting River School District as the site for my study, I could benefit the district in a number of ways. First, the results of this study illuminate the strengths of those science teachers who serve as cooperating teachers in the district. Understanding these teachers' perceived strengths could inform the design of targeted professional development that builds on those strengths. Second, teacher preparation programs in the Portland Metro Area could use the results of this study to design additional iterations of strategic clinical practice experiences for teacher candidates who conduct their student teaching in River School District. For example, the school district and teacher preparation programs could work together to place teacher candidates more strategically with cooperating teachers based on their perceived strengths. Third, the results of this study could add to the knowledge base of the district to contribute to River School Districts goal of serving those students who are traditionally underserved learners in STEM. Finally, in addition to the benefits to River School District specifically, the findings from this study can benefit the state of Oregon. The Oregon Department of Education can use the results to create policies that highlight those teachers who work with teacher candidates during their clinical practice experience.

Within River School District, I purposefully selected participants: science teachers who serve or have recently served (in the past five years) as a cooperating 
teacher hosting a science teacher candidate from any of the teacher preparation programs in Oregon. Based on the data available from River School District, I estimated that the population I could sample would be approximately 30-40 cooperating teachers. Within this sample, I aimed for a survey completion rate of approximately 30\% (Fowler, 2009) meaning I hoped to have survey results from 10-12 participants. After obtaining permission from the district to conduct my research, I learned that I had unfortunately overestimated the sample size. The district had record of 20 people who were either currently serving or had in the past five years served as a cooperating teacher hosting a science teacher candidate. Despite having overestimated the original sample size, fortunately, I had underestimated the completion rate of $30 \%$. With the TOSA's persistent efforts in communicating with the cooperating teachers, we achieved a $60 \%$ completion rate, and I gathered survey results from 12 cooperating teachers.

\section{Procedures}

Having obtained approval from both my dissertation committee and the IRB to conduct my study, I requested permission from River School District as the prospective school district to survey cooperating teacher participants. After receiving permission from River School District to conduct my study, I worked with the TOSA in the district to distribute survey information using a secure online survey platform (i.e., Qualtrics) as well as the details of informed consent (see Appendix D), to school email addresses of the 20 identified district cooperating teachers. In piloting the survey for my study (Culturally Sustaining Science Teaching Preparedness Survey), I learned that it would take cooperating teachers approximately 15-20 minutes to complete the full survey, 
including quantitative and qualitative components as well as the demographics section. The survey completion data showed that there was a range in time that it took the cooperating teachers to complete the survey from approximately four minutes (for those that completed only the quantitative components and demographic information) to 19 minutes (for those that complete the full survey). Throughout the time between the initial email and initial desired survey completion date, I checked the survey completion rate regularly on Qualtrics and communicated with the TOSA about progress. As the date for desired survey completion approached, I worked with the district TOSA to send additional requests to the cooperating teacher participants asking them to complete the survey. In response to participation numbers, I extended the completion date by two additional weeks, including one week that was spring break for the cooperating teachers. I completed the survey data collection at the end of March 2019.

After that time, I closed the survey and transferred the survey data in codedconfidential format to processing software (i.e., Excel), and saved the survey data on a password-protected computer. In April 2019, I completed initial analysis of both the coded-confidential quantitative and qualitative survey data and set up a time to share my preliminary findings with a focus group of those participants who indicated their willingness to be a part of the focus group on the survey. The use of a focus group fit into my convergent mixed methods design because it served as a way to strengthen the validity of my interpretation of the survey data. The focus group was not a separate data collection and analysis process. Recruitment of focus group participants turned out to be more of a challenge than I anticipated. While participation in the focus group interview 
was voluntary and took only approximately an hour of the cooperating teachers' time, I was only able to recruit two participants. While two participants are not a typical focus group size, I decided to conduct the focus group interview with these two participants for two reasons. First, the purpose of the focus group interview was to strengthen my interpretation of the survey data and not to gather a new source of data. Second, the two focus group participants represented a range of feelings and experiences of preparedness. I acknowledge that having only the two focus group participants is a limitation to my study; one that I address further in Chapter 4.

I met the two focus group participants at the city library because it was a central location for them. I conducted the focus group interview that took a full hour. With the permission of the participants, I used a recording device to record the interview. Then, I transferred the audio recording from the device to my password-protected computer so that I could transcribe the interview. After transcribing the focus group interview at the end of April 2019, I had collected and corroborated all the data (Merriam \& Tisdell, 2016). During summer 2019, I compiled the results and wrote a final draft of my research study.

\section{Instruments and Measures}

The survey instrument used for this study was a modification of Hsiao (2015) Culturally Responsive Teacher Preparedness Scale (see Appendix A). The modified version, Culturally Sustaining Science Teaching Preparedness Survey (adapted from Hsiao, 2015) (see Appendix B) includes a six-point Likert scale as the main tool of quantitative data collection as well as open-ended prompts as a tool for qualitative data 
collection. I chose to use and modify Hsiao's (2015) Culturally Responsive Teacher Preparedness Scale for a number of reasons. First, it has the capacity with some modification to measure the variables of concern (dependent variable $=$ cooperating teachers Likert-scale rating of components of the CSST conceptual framework on an ordinal six-point scale, independent variable $=$ categorical component of the CSST conceptual framework) within the outlined CSST conceptual framework. Second, Hsiao (2015) showed in an exploratory study that the survey had validity based on psychometric analysis and testing fairness for race and gender.

The results indicated that scores between male students and female students on the scale were not significantly different $\left(M_{\text {male }}=83.15, S D_{\text {male }}=14.84, n=27\right.$, vs. $\left.M_{\text {female }}=83.32, S D_{\text {female }}=14.43, n=160, \mathrm{t}=-.06, \mathrm{p}=.96\right)$. In addition, scores between white students and non-white students were not significantly different

$$
\begin{aligned}
& \left(M_{\text {white }}=83.25, S D_{\text {white }}=14.93, \mathrm{n}=141, \text { vs. } M_{\text {non }}=83.24, S D_{\text {non }}=13.12, n=45, \mathrm{t}\right. \\
& =.00, \mathrm{p}=.99) .(\text { Hsiao, } 2015, \text { p. } 246)
\end{aligned}
$$

I acknowledge that the modifications made to the scale for my studies survey may have an effect on the validity. Third, Hsiao is an emerging female scholar of color who I want to support, especially because scholars of color are often underrepresented in the literature.

I had permission from Hsiao to modify and use the scale to be a part of the survey for my doctoral research (see Appendix C). I modified the scale language to include CSP extensions of the CResP constructs Hsiao outlined that aligned with the components of my CSST conceptual framework. For example, I added language to the scale regarding 
obtaining, evaluating, and communicating information about systems of power and privilege because that aligns to CSP within the curriculum components of my CSST conceptual framework. I also adjusted the scale language for use with a range of agents in the teacher preparation network, including cooperating teachers rather than just teacher candidates (Hsiao, 2015). Based on feedback from piloting the Culturally Sustaining Science Teaching Preparedness Survey (adapted from Hsiao, 2015) and my dissertation committee, I adapted Hsiao's (2015) six-point Likert-scale - adding a descriptor for each level of the scale (Hsiao had only included qualifiers at the one and the six). Using a sixpoint scale that eliminates the neutral option, the participants had to choose between the levels of preparedness (Krosnick \& Presser, 2010). Additionally, I added open-ended prompts in the survey to gather qualitative data for my convergent mixed methods research.

While Hsiao’s (2015) Culturally Responsive Teacher Preparedness Scale was validated based on psychometric analysis and testing fairness for race and gender, there are some potential threats to that validity (both external and internal) as well as threats to reliability within my study design. I attempted to mitigate these threats through intentional planning and framing the research questions within the pragmatic paradigm. To attempt to mitigate external validity and reliability issues that could have arisen due to the modifications made to the survey; I acted before and after data collection and analysis. Before data collection, I piloted the Culturally Sustaining Science Teaching Preparedness Survey (adapted from Hsiao, 2015) with teacher educators that work with science teacher candidates outside the scope of my study. According to Creswell (2014), 
conducting pilot studies can help researchers to find out if their tools allow them to gather the information they intend to gather to answer their research questions. The pilot study allowed me to refine and clarify the language of the survey by eliminating redundant terms and providing more specific terms.

Following data collection and analysis of the Culturally Sustaining Science Teaching Preparedness Survey (adapted from Hsiao, 2015), I conducted a focus group with participants who indicate their willingness to be a part of the focus group on the survey. During this focus group, I presented my preliminary findings for feedback and corroboration. I only asked the focus group participants about the aggregate data on the completed and analyzed survey. I used data from the focus group interview to confirm and strengthen my interpretations of the survey data as well as to improve the validity and reliability of my overall findings.

To attempt to mitigate the potential internal threats to validity and reliability from using the survey instrument - the self-selection and self-report methods of data collection with the survey (Fowler, 2009) — I framed my research questions as expressed perceptions and approached the study through the pragmatic paradigm. Using a selfselect and self-report method on the survey meant there was a risk that only those who have either strong positive or negative feelings about their preparedness to support science teacher candidates to use CSP may choose to complete the survey (Creswell, 2014), thus skewing the data. For my study, it turned out that cooperating teachers with a range of feelings about their preparedness, including moderate feelings, chose to complete the survey. I discuss these findings more in the quantitative results section in 
Chapter 4. Even having cooperating teachers with a range of perceived feelings of preparedness choosing to complete the survey could not eliminate potential internal threats to validity and reliability. There was still the possibility that those who participated reported "social desirability rather than with their real ability" (Hsiao, 2015, p. 247). Framing the research questions as perceived preparedness and approaching the methodology from the pragmatic paradigm allowed me to mitigate these threats to the validity and reliability. As described in the Chapter 2 review of methods literature, researchers adopting a pragmatic paradigm acknowledge the social construction of knowledge (Morgan, 2014). Thus, I see this research not as understanding the 'objective ability' of teacher educators to support science teacher candidates to use CSP, but rather as a constructed understanding of their preparedness through their views.

\section{Role of Researcher}

I acknowledge that the procedures outlined for this study can put me, as the researcher, in a potential position of power relative to my participants (Smith, 2005), particularly because I am asking them to reveal information related to their professional practice, including ways they may not feel prepared. As the researcher, I am a teacher educator in a different role, the previously discussed often-privileged role of assistant professor that works with teacher candidates at the university level. As a White female researcher, I am in many ways operating from a place of privilege and power (Smith, 2005). Though my current role could place me, as the researcher, as an outsider, I possess some insider status (Merriam \& Tisdell, 2016) because I am also a previous classroom science teacher who hosted science teacher candidates in my classroom as a 
cooperating teacher. Regardless of my role and positionality, it was my responsibility throughout the study to maintain ethical research practices and consider ways to minimize risks to the participants.

I acknowledge that asking participants to reveal parts of their professional practice had the potential risk for minor psychological discomfort. To address the power differential and potential for minor psychological discomfort, I framed the research in a way that emphasizes the strengths expressed by the participants. I did not pressure participants into signing consent (Creswell, 2014) to complete the survey. Via email, I presented the participants with comprehensive information explaining the survey and directions for completing it should they choose to participate. Finally, I respected and guarded the privacy of the participants (Creswell, 2014) by keeping all information gathered in the survey and focus group confidential and secure. I promptly translated all survey data to a coded-confidential format. Then, I asked the focus group participants only about the aggregate analysis of the survey. Asking the focus group participants only about the aggregate analysis of the survey allowed me to not only member check my preliminary analysis but also served to safeguard focus group participants' confidentiality. Throughout all data collection and analysis, including the focus group, I kept participant information coded and confidential. I removed all identifiable information, including codes, when writing up the results.

\section{Data Collection and Analysis}

Because this was a convergent mixed methods study, data collection happened in a parallel fashion with both quantitative and qualitative data collected via the Culturally 
Sustaining Science Teaching Preparedness Survey (adapted from Hsiao, 2015)

instrument. I strengthened the interpretation of the aggregate survey data through the focus group interview. As mentioned previously, I called upon a validating quantitative data convergent mixed methods approach for my study not only because these methods aligned with the goals of my study but also because other researchers, Haag and Megowan (2015) and Thomas-Alexander and Harper (2017), had used and justified this parallel data collection process in their mixed methods studies. To address Research Question 1a, as shown in Table 1, the survey instrument collected quantitative data for each component of the CSST conceptual framework (curriculum, instruction, relationships) with a six-point Likert scale through the prompt "I am prepared to support science teacher candidates to..." with specific constructs following. To address Research Question 1b, as shown in Table 1, the survey instrument collected qualitative data through the open-ended prompts. Mirroring Thomas-Alexander and Harper (2017) methods of starting with a broad open-ended prompt, the first open-ended prompt was "In what ways do you feel prepared to support science teacher candidates to use pedagogies that are culturally sustaining for traditionally underserved learners." I intentionally did not define culturally sustaining pedagogies at this point in the survey as a way to let the participants speak into this prompt without influence as Thomas-Alexander and Harper (2017) did in their study. I defined culturally sustaining pedagogies within the Likertscale items, which represented the constructs of the conceptual framework components. To follow up on the Likert-scale items, I used open-ended prompts including, "Please describe your thoughts about the ratings you selected within the (curriculum, instruction, 
or relationships) section." I also used the survey to collect specific demographic information about the cooperating teacher participant experiences such as subjects taught, years serving as a cooperating teacher, and classroom student demographics. The focus group interview served as a tool to corroborate and strengthen my initial analysis of the survey data (see Appendix E for focus group interview questions).

Table 1

Research Question and Data Source Alignment

\begin{tabular}{lll}
\hline & \multicolumn{2}{c}{ Data Sources } \\
\hline Research Question 1 & $\begin{array}{l}\text { Survey Quantitative } \\
\text { Validate interpretations with } \\
\text { Focus Group Interview } \\
\text { Survey Quantitative } \\
\text { (Likert-scale items) }\end{array}$ & $\begin{array}{l}\text { Survey Qualitative } \\
\text { Validate interpretations } \\
\text { with Focus Group Interview }\end{array}$ \\
Research Question 1b & & $\begin{array}{l}\text { Survey Qualitative } \\
\text { (Open-ended responses) }\end{array}$ \\
\hline
\end{tabular}

According to Creswell and Plano-Clark (2007), threats to validity and reliability arise in the data collection process of convergent mixed methods designs when there are different sample sizes or different participants in the sample for the quantitative and qualitative segments of data collection. While I attempted to mitigate these threats to validity by embedding the open-ended qualitative survey prompts into the same survey with the Likert-scale quantitative items thus collecting both the quantitative data and qualitative data from the same participants and the same sample size it did not work out exactly as planned, as research rarely does. I did end up collecting data from the same participants, helping to shore up the validity because this was a validating quantitative data mixed methods study, but I did not end up with the same sample size of quantitative 
and qualitative data. I discuss these differences in sample size and the limitations of the sample size in Chapter 4.

Another threat to validity and reliability in data collection is the potential to introduce bias in the process. This could be especially true because of the potential for power differential between me, as the researcher, and the participants. I attempted to mitigate this threat, as suggested by Creswell and Plano-Clark (2007), by using the least obtrusive data collection process possible and selecting participants in which my interaction with them in the data collection process includes little to no power dynamics. All segments of the survey were included in one main communication with the participants via their school email address. The participants could complete the survey on their own time at their convenience. The only additional communication with participants was requests from the River School District TOSA to complete the survey if they have not already, and follow up from me about participation in the focus group if participants indicated a willingness to do so on the survey.

After collecting the quantitative and qualitative data from the survey, I analyzed the data sets for Research Question 1a and Research Question 1b separately. I conducted descriptive analytic statistics and non-parametric Mann-Whitney test procedures (Field, 2018) to analyze the quantitative data. I used a two cycle coding process (holistic and priory thematic) (Guest, MacQueen, \& Namey, 2012; Miles, Huberman, \& Saldaña, 2014; Saldaña, 2013) to analyze the qualitative data. After analyzing the quantitative and qualitative data separately, I merged the data to address overarching Research Question 1 (Creswell, 2014): with the qualitative data serving to validate the quantitative findings. 
When converging the data, I also used the focus group interview data to strengthen my interpretation of the findings.

I calculated descriptive statistics (mean and standard deviation) for each of the CSST conceptual framework component Likert-scales as well as for the Likert-items associated with the constructs of the components of the conceptual framework. The Likert-items were analyzed only with the descriptive statistics as it was not appropriate to run more sophisticated statistical analysis on these items (Boone \& Boone, 2012). For example, the construct-based Likert-items such as "1.1 I am currently to support science teacher candidates to... evaluate science curricula and instructional materials to determine their multicultural strengths and weaknesses, relevance to students' interests and instructional needs, and revise them if necessary" on the survey were analyzed using only mean and standard deviation (Boone \& Boone, 2012). In their study of sources of teacher candidates culturally responsive teaching self-efficacy doubt, Siwatu, Chesnut, Alejandro, and Young (2016) used a similar approach to quantitative analysis running descriptive analytics as a way to identify specific areas of need indicated on Likert-item type questions related to culturally responsive teaching efficacy.

I analyzed the components of the conceptual framework with more sophisticated statistical tests. Specifically, along with descriptive statistics in my study, I used a MannWhitney test - a variance on the tests used by Haag and Megowan (2015) and ThomasAlexander and Harper (2017) in their analyses - to determine if there were any statistically significant differences in the means on the component Likert-scales (Carifio 
\& Perla, 2008), indicating particularly strong expressions of preparedness. According to Field (2018):

...the Mann-Whitney test works by looking at differences in the ranked positions of scores in different groups...the Mann-Whitney test relies on scores being ranked from lowest to highest; therefore the group with the lowest mean rank is the group with the greatest number of low scores in it. Conversely, the group that has the highest mean rank should have a greater number of high scores within it. (p. 220)

I selected the Mann-Whitney test as an appropriate statistical analysis tool for my study because it is suitable for comparing group mean but does not rely on the assumption of normal distribution. Thus, the Mann-Whitney test was fitting as a statistical analysis tool when working with smaller sample sizes $\mathrm{n}<20$ (Field, 2018) such as the quantitative data sample size of my study. I used the Mann-Whitney test to compare the means of the Likert-scales associated with the combination of the Likert-item constructs into the three components of the conceptual framework (curriculum, instruction, relationships). For example, the component Likert-scales for the curriculum component as it compared to the instruction component were analyzed using mean, standard deviation, and the MannWhitney test to check for statistical significance in any difference that showed up in the descriptive statistics (Carifio \& Perla, 2008). I not only used the Mann-Whitney test to compare the overall means and check for statistically significant difference among the conceptual framework components overall, but I also used the test for each of the demographic components such as years teaching science, grade level taught, years as a 
cooperating teacher, and student demographics of their most diverse class. I calculated the Mann-Whitney mean rank, U-value, and $p$-value for the quantitative data portion of the analysis, which I report in Chapter 4.

I analyzed the qualitative data using holistic and then priory thematic coding that mirror quite closely the qualitative analysis Thomas-Alexander and Harper (2017) used but within my CSST conceptual framework. According to Guest, MacQueen, and Namey (2012), a thematic analytic approach to data analysis, rather than being focused on one methodological camp (such as realism or constructivism) focuses on how the techniques are applied and above all "ensuring the credibility of findings to an external audience" (p. 15). Thus, this approach to the focused qualitative data analysis was appropriate for my study, situated in the pragmatic paradigm that aimed to identify teacher educators' current perceived strengths with the qualitative data serving to validate the quantitative data. Specifically, I followed Saldaña (2013) two cycle coding process.

In two cycle coding, the first round allows a number of strategies for assigning codes to the data set (Saldaña, 2013). During the first round of coding, I used holistic coding as a way to assign codes to large chunks of the data (Miles et al., 2014) to identify broad themes before conducting a more detailed second round of coding using those themes. I developed the following themes from the first round of coding: preparation through professional development, acquiring skills on the job, and learning from colleagues and students. I then moved to focused thematic coding, with the components of the conceptual framework along with the themes identified during the first round of coding serving as the guide to assign codes during this second round. I coded statements 
having to do with acquiring preparedness on the job or acquiring preparedness by learning from colleagues or students around curriculum, instruction, or relationships during the second coding round. For example, I assigned codes such as "C-OTJ" or "RLCS" during this round. Finally, I examined and grouped the codes from the second round for similarities and differences. For example, if a statement assigned "C-OTJ" was about a specific strategy acquired on the job, I grouped those by "on the job." Whereas if a statement assigned "R-LCS" was about the importance of learning from a colleague or student within that component, I placed those into another group.

Having completed a preliminary analysis of the quantitative and qualitative survey data separately, I then took those findings to participants in the focus group interview for corroboration of my interpretation of the results. In the focus group interview I asked participants questions about the CSST components and constructs within those components that were identified as particular strengths or needs and how those matched (or not) with their understanding of the strengths and needs of cooperating teachers in their district. I also asked the focus group participants about the CSST themes identified in the open-ended prompts. The focus group interview served as a tool to strengthen my final analysis of converging the quantitative and qualitative findings to address the overarching research question.

As a final step in analysis, I merged the initial analysis of the two data sets to extend the findings "peering more deeply into the data so as to surface more nuanced understandings" (Goodwin et al., 2014, p. 287). I considered the descriptive analytic and Mann-Whitney quantitative results together with the qualitative results and the data from 
the focus group interview. Specifically, using the validating quantitative data approach (Creswell \& Plano-Clark, 2007), the grouping of coded statements was used as support for quantitative findings and to provide a rich description of cooperating teacher participants' strengths with respect to their preparedness to support science teacher candidates to use pedagogies that are culturally sustaining for their traditionally underserved learners.

According to Creswell and Plano-Clark (2007), in addition to the threats to validity and reliability that arise in mixed methods data collection, threats to validity and reliability can arise and should be minimizing during the data analysis process. The threats that were particularly relevant to my study are the threat of "inadequate data transformation approaches...inadequate approaches to converging the data" (p. 147). In an attempt to minimize these threats, I selected two studies as models to mirror the methods that were useful as a guide for the merging of the quantitative and qualitative data results.

In this chapter, I outlined the procedures for data collection and analyses that I used to conduct this study. I described the participants and their selection. I also described my role as the researcher. I explained the instruments and measures used in the study and justified their use. Additionally, I examined the threats to validity and reliability with respect to the data collection and analysis methods I outlined. I acknowledge that the procedures and methods outlined in this chapter have their limitations and I discuss these limitations in detail in the next chapter, Chapter 4, after I present the findings of the analysis to address each of the research questions. Despite 
limitations, I remain confident that the methods I used allow me to address the research questions and thus the purpose of this study, adding to the literature on preparing science teacher candidates to serve traditionally underserved learners in STEM. 


\section{Chapter 4: Results}

The problem of practice guiding my study was that within the shifting landscape of STEM education, too few science teachers are prepared to implement the new standards in ways that are culturally sustaining for their traditionally underserved learners. The purpose of this mixed methods study was to describe teacher educators'specifically cooperating teachers - perceived preparedness to support science teacher candidates to use culturally sustaining pedagogies by addressing the following research questions:

Research Question 1: How do cooperating teachers perceive their preparedness to guide science teacher candidates to use the components (i.e., curriculum, instruction, relationships) of culturally sustaining science teaching?

Research Question 1a: To what degree do cooperating teachers feel prepared to support science teacher candidates to use the components of culturally sustaining science teaching? (Quantitative)

Research Question 1b: How do cooperating teachers describe their preparedness to support science teacher candidates to use the components of culturally sustaining science teaching? (Qualitative)

In this chapter, I share the findings from my collection and analysis of data from the Culturally Sustaining Science Teaching Preparedness Survey (adapted from Hsiao, 2015). First, I present the demographic information about the study participants. Then, I present the analysis and interpretation of the quantitative survey data to address Research Question 1a. Next, I report the analysis and interpretation of the qualitative data of the 
survey to address Research Question 1b. Finally, I converge the two data sets and interpret them in light of the focus group interview to strengthen my overall interpretation of the results to address the overarching Research Question 1.

\section{Participant Demographics}

The 12 participants were science teachers in the River School District who currently serve or have served (in the past five years) as cooperating teachers who host science teacher candidates. All 12 participants completed all of the quantitative items (i.e., Part 1: Culturally sustaining science teaching item ratings) on the Culturally Sustaining Science Teaching Preparedness Survey (adapted from Hsiao, 2015). Eleven of the 12 participants completed the demographic items (i.e., Part 2: Experience as a teacher educator) on the survey (see Table 2). The survey participants were middle and high school teachers with a range of years of experience teaching science, a range of years as a cooperating teacher, as well as a variety of subjects taught as a cooperating teacher. A majority (7 of 11) of the participants who completed the demographic items reported having $50 \%$ or greater students of color in their class.

Table 2

Quantitative Survey Data Participants' Demographic Factors $(n=11)$

Demographic factor $\quad$ Category and number of cooperating teachers

Years of teaching science

Grade level

Years as a cooperating teacher
10 years or greater $n=8$

Less than 10 years $n=3$

Middle school $n=3$

High school $n=8$

$\begin{aligned} 1-3 n & =6 \\ 4-6 n & =4 \\ 6-8 n & =0\end{aligned}$ 
$10-12 n=1$

Demographics of most diverse class

Subjects taught as cooperating teacher
$50 \%$ or greater students of color $n=7$ Less than $50 \%$ students of color $n=4$

General science, Environmental science, Biology, Chemistry, Physics, Integrated science

Of the 12 participants that completed all the quantitative aspects of the survey, six completed the qualitative aspects (i.e, Part 1: Culturally sustaining science teaching openended responses) of the Culturally Sustaining Science Teaching Preparedness Survey (adapted from Hsiao, 2015) and four of those six provided substantive qualitative data (see Table 3). The survey participants who provided substantive qualitative data were both middle and high school teachers, the majority of who (3 of 4) reported having more than 10 years of teaching experience but only 1-3 years of experience as a cooperating teacher. The other two qualitative survey responses were too short or incomplete, so I did not include them. For example, in response to the open-ended prompt, "In what ways do you feel prepared to support science teacher candidates to use pedagogies that are culturally sustaining for traditionally underserved learners?" One participant wrote, "I feel somewhat prepared to support." I did not consider these types of responses to be substantive enough to be included in the qualitative analysis. Thus, the qualitative data analysis consists of responses from four participants. Because I designed this mixed methods study as a validating quantitative data model, the purpose of the qualitative data was to validate and expand upon the quantitative findings (Creswell \& Plano-Clark, 2007). Interpreting the qualitative data from these four survey participants was justifiable 
and within the goals of the study, particularly because these participants represented a variety of degrees of expressed preparedness as well as a variety of demographic factors. However, I acknowledge that having qualitative survey data from only four of the 12 survey completers was one of the limitations of this study, which I discuss later in this chapter.

Table 3

Qualitative Survey Data Participants' Demographic Factors $(n=4)$

Demographic factor $\quad$ Category and number of cooperating teachers

Years of teaching science

10 years or greater $n=3$

Less than 10 years $n=1$

Grade level

Middle school $n=3$

High school $n=1$

Years as a cooperating teacher

$$
\begin{aligned}
& 1-3 n=3 \\
& 4-6 n=1 \\
& 6-8 n=0 \\
& 10-12 n=0
\end{aligned}
$$

Demographics of most diverse class

Subjects taught as cooperating teacher
$50 \%$ or greater students of color $n=1$

Less than $50 \%$ students of color $n=3$

General science, Biology, Chemistry, Integrated science

Also relevant to my overall data analysis was the focus group data that came from two participants (see Table 4). Having only two participants in the focus group interview was due to scheduling difficulties (e.g., busy professionals with competing demands on their time). One focus group participant was a middle school teacher and the other was a high school teacher. Both reported more than 10 years of teaching experience and 
student demographics of $50 \%$ or greater students of color in their most diverse class.

While two is not a traditional size for a focus group, the purpose of my focus group interview was to strengthen my interpretation of the survey data and not to gather another source of data. Similar to those who provided substantive qualitative data, the two focus group participants represented a range of perceived preparedness and demographic factors. Therefore, I proceeded to use the data from the focus group interview as planned - to strengthen my interpretation of the survey data. Nevertheless, I acknowledge that having only two focus group participants is another limitation of the study, which I discuss at the end of this chapter.

Table 4

Focus Group Participants’ Demographic Factors $(n=2)$

Demographic factor Category and number of cooperating teachers

Years of teaching science $\quad 10$ years or greater $n=2$

Less than 10 years $n=0$

Grade level

Middle school $n=1$

High school $n=1$

Years as a cooperating teacher

$$
\begin{aligned}
& 1-3 n=1 \\
& 4-6 n=1 \\
& \text { 6-8 } n=0 \\
& 10-12 n=0
\end{aligned}
$$

Demographics of most diverse class

$50 \%$ or greater students of color $n=2$

Less than $50 \%$ students of color $n=0$

Subjects taught as cooperating teacher

General science, Biology, Chemistry, Physics

\section{Presentation and Analysis of Survey Quantitative Data}


To share the results of the quantitative analysis, I begin by presenting the descriptive statistics and then report the results of a series of three Mann-Whitney tests conducted on the quantitative survey data. As a review, the following represents that culturally sustaining science teaching (CSST) conceptual framework.

- The science teacher candidate is prepared to develop culturally mediated curriculum that includes students' cultural identities (Gay, 2010) and real world connections to students lived experiences including students obtaining, evaluating, and communicating information (Brown, 2017) about systems of power and oppression in science (Paris \& Alim, 2017).

- The science teacher candidate is prepared to facilitate learner-centered instruction that promotes agency and input from all students (Gay, 2010) and centers on collective and dynamic community languages as assets (Paris \& Alim, 2017) to learning as students develop and use models that represent a broader understanding of science concepts (Brown, 2017).

- The science teacher candidate is prepared to foster relationships of dignity and care (Paris \& Alim, 2017) grounded in positive perceptions that communicate high expectations to all students within a collaborative learning community (Gay, 2010) where students work together to construct explanations and designing solutions to problems or challenges (Brown, 2017). 
Table 5 lists the components and constructs of the conceptual framework as they were organized on the Culturally Sustaining Science Teaching Preparedness Survey (adapted from Hsiao, 2015) and analyzed for my study.

Table 5

Components and Constructs of Conceptual Framework

Components Constructs

Curriculum

1.1. evaluate science curricula and instructional materials to determine their multicultural strengths and weaknesses, relevance to students' interests and instructional needs, and revise them if necessary.

1.2. develop a repertoire of examples in the science curriculum that are culturally familiar to students to scaffold learning.

1.3. infuse the science curriculum, including units and lessons, with the culture of students represented in the classroom.

1.4. include a variety of instructional methods to match students' learning preferences, and maintain their attention and interest in science.

1.5. design science curriculum that includes students obtaining, evaluating, and communicating information about systems of power, privilege, and marginalization.

Instruction

2.1. find ways to enhance culturally and linguistically diverse students' comprehension and use of science related content, concepts, vocabulary, and skills.

2.2. use a variety of linguistic styles with culturally diverse students in an attempt to communicate in culturally responsive or sustaining ways during science instruction.

2.3. create a community of learners by encouraging students to focus on collective work, responsibility, and cooperation when learning science.

2.4. provide students with knowledge and skills needed to function in mainstream culture of science and to consider the ways various cultural groups, including their own contribute to science.

2.5. assist students in developing and using models that represent various ways of knowing science based on their cultural practices and knowledge.

Relationships 3.1. create a warm, supporting, safe, and secure classroom 
environment for culturally diverse students to learn science.

3.2. develop and maintain positive, meaningful, caring, and trusting relationships with students.

3.3. establish expectations for appropriate classroom behavior in considering students' cultural backgrounds to maintain a conducive learning environment.

3.4. communicate expectations of success to culturally diverse students that are grounded in positive perceptions of all learners.

3.5. guide students to construct explanations about problems or challenges that impact them and their communities.

For this analysis, the components of the CSST conceptual framework (curriculum vs. instruction, instruction vs. relationships, relationships vs. curriculum) are the independent variables. The participants' rating of construct Likert-items compiled into component Likert-scales ranging from "unprepared" to "fully prepared" are the dependent variables. Because I was examining the data from the categorical independent variable of CSST framework components and the ordinal dependent variable of Likertscale rating, I used the Mann-Whitney as a non-parametric statistical test. The MannWhitney test is a variation of the independent t-test suitable for smaller sample sizes and was the best-fit statistical model for analysis for my study. I selected the Mann-Whitney test rather than the Wilcoxon signed-rank test, another commonly used statistical model for smaller sample sizes comparing means, because I wanted to compare the mean score ranks of the CSST framework components at a certain point in time and under the same conditions - rather than comparing how mean scores changed over time based on an intervention (Field, 2018). I used the Mann-Whitney statistical model (Field, 2018) to determine if the CSST framework components would have statistically significant difference in mean score ranks on the Likert-scale ratings. I hypothesized that the Likert- 
scale ratings would have different mean score ranks for the different CSST framework components, though I did not have a claim about which component would outperform the other (Field, 2018).

As a first step in determining whether the participants expressed differences in their perceived preparedness on any of the components of the CSST conceptual framework, I calculated descriptive statistics (see Table 6).

Table 6

Descriptive Statistics for Quantitative Survey Data: Component and Construct $(n=12)$

\begin{tabular}{ccc}
\hline $\begin{array}{l}\text { Conceptual framework } \\
\text { components and constructs }\end{array}$ & Mean & Standard Deviation \\
\hline Curriculum & 4.15 & \\
1.1 & 4.33 & 1.03 \\
1.2 & 3.92 & 1.03 \\
1.3 & 3.92 & 1.19 \\
1.4 & 4.83 & 1.38 \\
1.5 & 3.75 & 0.90 \\
& & 1.42 \\
Instruction & 4.10 & \\
2.1 & 4.08 & 1.01 \\
2.2 & 3.67 & 1.19 \\
2.3 & 4.75 & 1.37 \\
2.4 & 4.08 & 1.01 \\
2.5 & 3.92 & 1.04 \\
& & 1.32 \\
Relationships & 4.68 & \\
3.1 & 4.58 & 1.12 \\
3.2 & 5.08 & 1.26 \\
3.3 & 4.67 & 0.95 \\
3.4 & 4.50 & 1.11 \\
3.5 & 4.58 & 1.38 \\
& & 1.26 \\
Overall & 4.31 & \\
\hline
\end{tabular}


Because I was interested in comparing the mean scores of the various components of the CSST conceptual framework I started by observing those mean scores overall. One of the first things that I noticed was that the overall mean scores for each of the components were within the "prepared" qualifier or a score of between 4 and 5 on the survey Likert-scale. Suggesting that regardless of which component's mean scores were higher, all of the components mean scores seemed to indicate that the participants felt "prepared." While the relationships component was within the "prepared" qualifier $(M=$ 4.68), it was greater than the means of both the curriculum $(M=4.15)$ and instruction $(M$ $=4.10$ ) components, respectively. In other words, participants seemed to express a greater perceived preparedness to support teacher candidates to foster relationships of dignity and care with their students than they were expressing to support teacher candidates to develop culturally mediated curriculum or facilitate student-centered instruction. In noticing this difference, I also noticed a similarity between the mean scores for the curriculum and instruction components. There seemed to be essentially no difference in the way participants perceived their preparedness to support teacher candidates to develop culturally mediated curriculum and the way they perceived their preparedness to support teacher candidates to facilitate student-centered instruction. The little difference between cooperating teachers' preparedness for these components was also apparent in the standard deviations for curriculum $(S D=1.03)$ and instruction $(S D=$ 1.01). These standard deviations indicated that the differences between participants perceiving themselves to be the "most prepared" for these components and those perceiving themselves to be the "least prepared" for these components was similar. 
After looking at the patterns of the Likert-scale mean scores overall, I decided to look at the individual construct items of each of the components of the conceptual framework that comprise the Likert-scale score. I did not run statistical analyses on the differences between the constructs of each component because these are Likert-item scores not Likert-scales. According to Boone and Boone (2012), only more basic statistics analyses such as descriptive statistics are appropriate for interpreting Likertitems. Even though I did not run statistical tests on the individual constructs that made up the components of Likert-scale scores, it was helpful to look closely at the specific aspects of practice that the participants expressed perceived preparedness around.

Relationships. In looking at the individual constructs, I first noticed that all the constructs in the relationships component had a mean score equal to or greater than 4.5 $\left(M_{3.1}=4.58, M_{3.2}=5.08, M_{3.3}=4.67, M_{3.4}=4.5, M_{3.5}=4.58\right)$. The data indicated that not only do the participants perceive themselves to be prepared to support teacher candidates to foster relationships of dignity and care with their students overall but they feel prepared in all aspects of this component. The construct of the relationships component that they felt most prepared to support their teacher candidates was 3.2 "develop and maintain positive, meaningful, caring, and trusting relationships with students" $\left(M_{3.2}=\right.$ 5.08). This construct had the highest mean score of all the constructs not just those in the relationships component with an average slightly above a score of 5, the "highly prepared" qualifier on the survey Likert-scale. This construct not only had the highest mean score for the entire constructs but also one of the lowest standard deviations $(S D=$ 0.95). Indicating that not only did the participants on average feel "highly prepared" with 
respect to this construct, but there was little difference between those perceiving themselves the "most prepared" to support their teacher candidates with this construct and those that expressed the "least perceived preparedness" with this construct.

Curriculum. Second, I noticed that the curriculum component contained the construct with the second highest mean score of all the constructs 1.4 "include a variety of instructional methods to match students' learning preferences, and maintain their attention and interest in science" $\left(M_{1.4}=4.83\right)$. Not only did this construct have the second highest mean score it also had the lowest standard deviation of all the constructs $(S D=0.90)$. Indicating again, the differences between those participants perceiving themselves the "most prepared" to support their teacher candidates with this construct and those that expressed the "least perceived preparedness" was minimal. Although the curriculum component was in the "prepared" qualifier with the overall mean score between 4 and 5 on the survey Likert-scale, three constructs in that component were perceived by the participants as only "somewhat prepared" with mean scores between 3 and 4 on the scale $\left(M_{1.2}=3.92, M_{1.3}=3.92, M_{1.5}=3.75\right)$. This data indicates that for the curriculum component participants feel more prepared for some constructs of it than others. For example, the construct receiving the lowest mean score for the curriculum component was 1.5 "design science curriculum that includes students obtaining, evaluating, and communicating information about systems of power, privilege, and marginalization" $\left(M_{1.5}=3.75\right)$.

Instruction. Third, I noticed that the construct rated highest for the instruction component and third highest overall for all the constructs was 2.3 "create a community of 
learners by encouraging students to focus on collective work, responsibility, and cooperation when learning science" $\left(M_{2.3}=4.75\right)$. Despite being in the "prepared" qualifier overall with the mean score between 4 and 5 on the survey Likert-scale within the instruction component there were two constructs that were perceived by the participants as only "somewhat prepared" with mean score between 3 and 4 on the survey Likert-scale $\left(M_{2.2}=3.67, M_{2.5}=3.92\right)$. I noticed that the lowest construct in the instruction component was 2.2 "use a variety of linguistic styles with culturally diverse students in an attempt to communicate in culturally responsive or sustaining ways during science instruction" $\left(M_{2.2}=3.67\right)$ and it was even lower than the lowest mean score for the curriculum component. These construct results within the instruction component mirror the patterns indicated for the curriculum component constructs. While participants feel "prepared" overall for these components the participants vary in their perceived preparedness for the constructs within them.

As a final step in looking at the descriptive statistics, I looked at the individual mean score for the participants on all three components of the survey to notice patterns (see Table 7). While there seemed to be a range of expressed levels of individual preparedness, about half of the participants' (6 of 12) mean scores fell right near the "preparedness" qualifier on the survey Likert-scale with a mean score close to 4 .

\section{Table 7}

Individual Survey Participant Descriptive Statistics

\begin{tabular}{ccc}
\hline Participant & Mean & Standard Deviation \\
\hline A & 4.87 & 0.84 \\
B & 6.00 & 0.00 \\
C & 4.33 & 0.47
\end{tabular}




$\begin{array}{lll}\text { D } & 5.93 & 0.09 \\ \text { E } & 3.93 & 0.47 \\ \text { F } & 3.73 & 0.38 \\ \text { G } & 4.00 & 0.43 \\ \text { H } & 3.73 & 0.50 \\ \text { I } & 2.67 & 0.19 \\ \text { J } & 4.13 & 0.19 \\ \text { K } & 5.33 & 0.47 \\ \text { L } & 3.07 & 0.25\end{array}$

After reviewing the descriptive statistics for patterns (see Tables 6-7), I used a statistical model to determine if the observations I made about differences were in some way statistically significant. First, I ran Mann-Whitney tests comparing means for the CSST conceptual framework components. The Mann-Whitney test compares the means from two groups so I ran a series of three tests to compare: curriculum and instruction, curriculum and relationships, and instruction and relationships. The results of these Mann-Whitney tests varied (see Table 8).

Table 8

Mann-Whitney Test Results Comparing Conceptual Framework Components $(n=11)$

\begin{tabular}{lccc}
\hline $\begin{array}{l}\text { Conceptual framework } \\
\text { components and constructs }\end{array}$ & Mean Rank & $\begin{array}{l}\text { Mann-Whitney } \\
U \text { value }\end{array}$ & $p$-value \\
\hline Curriculum & 5.60 & 12.00 & 0.916 \\
Vs. Instruction & 5.40 & & \\
Curriculum & 3.80 & 4.00 & 0.074 \\
Vs. Relationships & 7.20 & & \\
Instruction & 3.80 & 4.00 & 0.074 \\
Vs. Relationships & 7.20 & & \\
\hline
\end{tabular}


The Mann-Whitney $U$ value indicates the relative difference or similarity in the distributions of the means ranks for the two groups being tests (Field, 2018). The $U$ value is based on the ranked position of scores, or mean ranks, from the two groups. The higher the $U$ value the closer those mean ranks are to one another, while a lower value indicates more difference in the distribution of those mean ranks. The $p$ value indicates the statistical significance of those similarities or differences. A higher $p$ value indicates more similarity and less significant differences, while a lower $p$ value indicates more significant differences with anything less than the $\alpha$-level of .05 being considered statistically significant. As shown in Table 8 , the mean score ranks including the $U$ and $p$ values from the Mann-Whitney tests showed that participants expressed higher levels of preparedness for the relationships component as compared to the curriculum and instruction components $(U=4.00)$. The Mann-Whitney tests also indicated that participants expressed similar levels of preparedness for the curriculum and instruction components $(U=12.00)$. The $p$-value comparing curriculum and instruction $(p=0.916)$ was greater than the $\alpha$-level of .05, so there is no statistically significant difference in participants' perceived preparedness between the curriculum and instruction components of the conceptual framework. The p-values comparing curriculum and relationships and that comparing instruction and relationships $(p=0.074)$ were both also greater than the $\alpha$-level of .05, so there is no statistically significant difference in participants' perceived preparedness between the relationships component and either the curriculum or instruction components of the conceptual framework. Due to how close the curriculum and instruction components were for mean score rank, it is not surprising that the $p$ - 
values were the same when comparing curriculum and relationships as they are when comparing instruction and relationships $(p=0.074)$. Because the $p$-values were all greater than the $\alpha$-level of .05 , there was no statistically significant difference between the levels of preparedness expressed by participants between any the components of the CSST conceptual framework. Despite the lack of statistically significant differences among the conceptual framework components, there were differences. The $p$ value for the difference in mean scores between curriculum and relationships and instruction and relationships were closer to the $\alpha$-level of $.05(p=0.074)$. Given how close this $p$-value comparing relationships to both curriculum and instruction is to a level of implication, especially in relation to the $p$-value comparing curriculum and instruction, I chose to explore these results with the focus group interview. Later in the chapter, I explain more about this exploration when discussing my overall interpretations of the findings using the focus group interview data to strengthen my interpretation.

After running the Mann-Whitney tests on the means for all participants' Likertscale scores on the components of the CSST conceptual framework overall and noting no areas of statistically significant difference, I wanted to know if there were any areas of significant difference in expressed preparedness with respect to the participants' demographic factors. To look into this, I ran Mann-Whitney tests comparing the participants' individual mean scores on the full Likert-scale (see Table 7) with the demographic factors including (a) student demographics (50\% or greater student or color versus less than 50\% students of color in classes), (b) grade level taught (middle school versus high school), (c) years as a science teacher (10 or great years teaching science 
versus less than 10 years teaching science), (d) years as a cooperating teacher (1-3 years versus 4-6 years). I conducted the analysis for these particular demographic factors because none of the participants had 6-8 years of experience as a cooperating teacher and only one participant had more than 10 years of experience (see Table 9).

Table 9

Mann-Whitney Test Results Comparing Teacher and Student Demographics for Conceptual Framework Overall $(n=11)$

\begin{tabular}{llll}
\hline $\begin{array}{l}\text { Mean comparisons, } \\
\text { Full Likert scale }\end{array}$ & Mean Rank & $\begin{array}{l}\text { Mann-Whitney } \\
U \text { value }\end{array}$ & $p$-value \\
\hline $\begin{array}{l}\text { 50\% or }>\text { students of color } \\
<50 \% \text { students of color }\end{array}$ & 6.36 & 11.50 & 0.636 \\
& 5.38 & & 0.609 \\
Middle school & 5.17 & 9.50 & \\
High school & 6.31 & & 0.306 \\
10 or $>$ years of teaching & 5.38 & 7.00 & \\
$<10$ years of teaching & 7.67 & & 0.748 \\
$\begin{array}{l}\text { 1-3 years as CT } \\
\text { 4-6 years as CT }\end{array}$ & 5.25 & 10.50 & \\
\hline
\end{tabular}

The results shown in Table 9 indicate that when comparing the individual participant's mean scores on the full Likert-scale to certain demographic factors that there were no statistically significant differences. All of the $p$-values were greater than the $\alpha$ level of .05. The demographic factor of years as a science teacher $(p=0.306)$ seemed to indicate more influence on participants expressed preparedness, compared to the other factors, but was not close enough to a level of significance to be interpreted as meaningful in the analysis. Having again noted no areas of statistically significant difference in the Mann-Whitney tests, I wanted to take my quantitative analysis further. 
Therefore, I ran Mann-Whitney tests comparing these same demographic factors with the participant's mean score on each individual CSST conceptual framework components, starting with the curriculum component (see Table 10).

Table 10

Mann-Whitney Test Results Comparing Teacher and Student Demographics for Curriculum Component of Conceptual Framework $(n=11)$

\begin{tabular}{llll}
\hline $\begin{array}{l}\text { Mean comparisons, } \\
\text { Curriculum }\end{array}$ & Mean Rank & $\begin{array}{l}\text { Mann-Whitney } \\
U \text { value }\end{array}$ & $p$-value \\
\hline $\begin{array}{l}50 \% \text { or }>\text { students of color } \\
<50 \% \text { students of color }\end{array}$ & 6.18 & 5.50 & 0.179 \\
$\begin{array}{l}\text { Middle school } \\
\text { High school }\end{array}$ & 7.03 & 7.00 & 0.633 \\
10 or $>$ years of teaching & 5.78 & & 0.301 \\
$<10$ years of teaching & 7.67 & 7.00 & \\
$\begin{array}{l}\text { 1-3 years as CT } \\
\text { 4-6 years as CT }\end{array}$ & 5.33 & & 0.829 \\
\hline
\end{tabular}

The results shown in Table 10 indicate that when comparing the individual participant's mean scores on the Likert-scale for the curriculum component to certain demographic factors that there were no statistically significant differences. All of the $p$ values were greater than the $\alpha$-level of .05 , and many were similar to the values computed for the components overall Likert-scale. One area of difference for the curriculum component was the demographic factor of student demographics. The $p$-value for curriculum ( $p=0.179$ ) was much smaller than it had been for this demographic factor overall $(p=0.636)$, indicating that the demographic factor of $50 \%$ or greater students of 
color could be more relevant for the curriculum component. Next, I calculated the demographic factors for the instruction component (see Table 11).

Table 11

Mann-Whitney Test Results Comparing Teacher and Student Demographics for Instruction Component of Conceptual Framework $(n=11)$

\begin{tabular}{lccc}
\hline $\begin{array}{l}\text { Mean comparisons, } \\
\text { Instruction }\end{array}$ & Mean Rank & $\begin{array}{l}\text { Mann-Whitney } \\
U \text { value }\end{array}$ & $p$-value \\
\hline $\begin{array}{l}50 \% \text { or }>\text { students of color } \\
<50 \% \text { students of color }\end{array}$ & 6.06 & 11.50 & 0.918 \\
& 5.83 & & \\
Middle school & 4.00 & 5.00 & 0.344 \\
High school & 6.44 & & 0.356 \\
& 5.44 & 7.50 & \\
10 or $>$ years of teaching & 7.50 & & 1.00 \\
$<10$ years of teaching & 5.50 & 12.00 & \\
1-3 years as CT & 5.50 & & \\
4-6 years as CT &
\end{tabular}

The results shown in Table 11 indicate that when comparing the individual participant's mean scores on the Likert-scale for the instruction component to certain demographic factors that there were no statistically significant differences. All of the $p$ values were greater than the $\alpha$-level of .05; however, similar to the curriculum component, there were notable areas of difference. For the instruction component the demographic factor of grade level taught $(p=0.344)$ appeared to be more influential than it was for either the overall components $(p=0.690)$ or the curriculum component ( $p=$ 0.633). Additionally, the $p$-value for the demographic factor of student demographics appeared to be less influential for instruction $(p=0.918)$ than it was overall $(p=0.636)$ 
or for curriculum $(p=0.179)$. As a final step in examining the demographic factors, I calculated the demographic factors for the relationships components (see Table 12).

Table 12

Mann-Whitney Test Results Comparing Teacher and Student Demographics for Relationships Component of Conceptual Framework $(n=11)$

\begin{tabular}{llll}
\hline $\begin{array}{l}\text { Mean comparisons, } \\
\text { Relationships }\end{array}$ & Mean Rank & $\begin{array}{l}\text { Mann-Whitney } \\
U \text { value }\end{array}$ & $p$-value \\
\hline $\begin{array}{l}\text { 50\% or }>\text { students of color } \\
<50 \% \text { students of color }\end{array}$ & 6.81 & 5.50 & 0.172 \\
& 3.83 & & \\
$\begin{array}{l}\text { Middle school } \\
\text { High school }\end{array}$ & 6.00 & 9.00 & 1.00 \\
$\begin{array}{l}\text { 10 or }>\text { years of teaching } \\
<10 \text { years of teaching }\end{array}$ & 6.00 & & 0.462 \\
1-3 years as CT & 7.17 & 8.50 & \\
4-6 years as CT & 4.75 & & 0.328 \\
\hline
\end{tabular}

The results shown in Table 12 indicate that when comparing the individual participant's mean scores on the Likert-scale for the relationships component to certain demographic factors that there were no statistically significant differences. All of the $p$ values were greater than the $\alpha$-level of .05 but like the curriculum and instruction components there were notable areas of difference shown. The $p$-value for relationships for the demographic factor of student demographics $(p=0.172)$ was much smaller than it had been for this demographic factor overall $(p=0.636)$ or for the instruction component $(p=0.918)$, but it was similar to that of the curriculum component $(p=0.179)$. Indicating that the demographic factor of $50 \%$ or greater students of color be more relevant for both the curriculum and relationships component. Additionally, the 
demographic factor of years as a cooperating teacher seemed to be more influential for the relationships component $(p=0.328)$ than it was for the overall components $(p=$ $0.690)$ or curriculum $(p=0.633)$ and instruction $(p=0.633)$. While the relationships components appeared to be more affected by the demographic factors of years as a cooperating teacher and student demographics, it seemed to be less influenced by the demographic factors of grade level taught and years as a science teacher. Although not statistically significant, these findings highlighted again the nuanced differences that exist in the participants expressed preparedness for the components of the CSST conceptual framework. In the next section, I discuss these differing $p$-values with the interpretation of the results, focusing on the most notable differences for the demographic factor of student demographics.

\section{Interpretation of Survey Quantitative Data}

In using convergent mixed methods design for the analysis and interpretation of the results, I interpreted the quantitative and qualitative data separately to address each of the sub-research questions and then converged the data to address the overarching research question in light of the focus group interview data to strengthen my interpretation. In this section, I interpret the quantitative survey data. I address the following sub-research question in this interpretation:

Research Question 1a: To what degree do cooperating teachers feel prepared to support science teacher candidates to use the components of culturally sustaining science teaching? (Quantitative) 
When looking at the results of the components of the conceptual framework overall, the mean scores for each component's Likert-scale indicate that participants $(n=$ 12) feel "prepared" to support science teacher candidates to use the components of the CSST framework (see Table 6). The mean scores for each component were roughly equivalent to or slightly higher than the "prepared" qualifier or between 4 and 5 on the survey Likert-scale. The relationships component $(M=4.68, U=4.00)$ ranked the highest degree of preparedness followed by the curriculum component ( $M=4.15, U=$ $12.00)$ and then the instruction component $(M=4.10, U=12.00)$, respectively. No statistically significant difference was shown from the Mann-Whitney tests $(n=11)$ comparing the mean score for each of these components (see Table 8) because the $p$ values were greater than the $\alpha$-level of $.05(p=0.916, p=0.074, p=0.074)$. Despite the lack of statistical significance between these components, there was a difference between the relationships component as compared to the curriculum and instruction component ( $p$ $=0.074)$. This difference suggests that participants feel even more prepared to support science teacher candidates to develop relationships or dignity and care with their students than they do to support them to develop culturally mediated curriculum or facilitate learner-centered instruction.

While I did not conduct a statistical analysis on the individual constructs within each component of the conceptual framework (see Table 5), I observed difference in the descriptive statistics worth noting in the interpretation of the quantitative survey findings (see Table 6). For the relationships component, all constructs within that component had a mean score of 4 or greater on the survey Likert-scale $\left(M_{3.1}=4.58, M_{3.2}=5.08, M_{3.3}=\right.$ 
$\left.4.67, M_{3.4}=4.5, M_{3.5}=4.58\right)$ ranking them as "prepared" or "highly prepared" on the survey qualifiers. These mean scores for the individual constructs of the relationships components indicate that no matter which construct I was examining within the relationships component, participants are expressing that they perceive themselves to be "prepared" or in one case even "highly prepared" to support their science teacher candidates to use those aspects of CSST. Yet, for the curriculum $(M=4.15)$ and instruction $(M=4.10)$ components while overall participants are expressing their preparedness at the degree of "prepared" at the individual constructs within these components the data indicates that participants are not perceiving their preparedness to the same degree. Within both the curriculum and instruction component, a few constructs for each component fall closer to the "somewhat prepared" qualifier with mean score between 3 and 4 on the survey Likert-scale $\left(M_{1.2}=3.92, M_{1.3}=3.92, M_{1.5}=3.75\right.$, $M_{2.2}=3.67, M_{2.5}=3.92$ ). For the curriculum component, in particular, the mean scores on individual constructs include both the highest construct $\left(M_{1.4}=4.83\right)$ and one of the lowest constructs $\left(M_{1.5}=3.75\right)$, indicating a variance in the degree to which participants perceived their preparedness to support their science teacher candidates to use these aspects of CSST.

Finally, to examine these differences more closely, I ran Mann-Whitney tests comparing the mean scores for the different components to demographic factors such as; (a) student demographics $(50 \%$ or greater student or color versus less than $50 \%$ students of color in classes), (b) grade level taught (middle school versus high school), (c) years as a science teacher (10 or great years teaching science versus less than 10 years teaching 
science), (d) years as a cooperating teacher (1-3 years versus 4-6 years). Again, I found no statistically significant differences among any of the components of the conceptual framework for any of the demographic factors explored. Worth noting, however, are the interesting differences in $p$-values in the interpretation of the quantitative survey results. Mainly, the student demographic of $50 \%$ or greater students of color versus less than $50 \%$ students of color seemed to be more meaningful for the curriculum $(p=0.179)$ and relationships component $(p=0.172)$ related to the overall $p$-value $(p=0.636)$ for these student demographics. However, for the instruction component, these student demographics seemed to be less meaningful $(p=0.918)$ related to the overall $p$-value for these student demographics. These results indicate that student demographics (e.g., percentage of students of color in the most diverse class) could have more effect on participants' perceived preparedness with respect to curriculum and relationships than the results for the instruction component. These results are particularly interesting given that the curriculum and instruction components are similar in this quantitative data, but they seem to converge in this one way. I see this as an area of potential future research, which I explain in Chapter 5.

\section{Presentation and Analysis of Survey Qualitative Data}

The purpose of the qualitative data in my convergent mixed methods study was to validate the quantitative data and to provide depth to my understanding of that quantitative data. Thus, having conducted a thorough analysis of the quantitative data, to begin analysis of the qualitative data, I conducted two rounds of coding (Saldaña, 2013). I started with holistic coding (Miles et al., 2014) to assign codes to chunk of the 
qualitative survey data to identify overarching themes that could guide the second round of coding. Through holistic coding of the qualitative survey data, I identified that although participants felt they were not originally prepared for CSST in their teacher preparation programs, they did feel prepared to support teacher candidates with such approaches now. The preparedness they expressed now was the result of what they learned through experience teaching or attending professional development. Specifically, I developed the following themes about their preparedness during the holistic round of coding: (a) preparation through professional development, (b) acquiring skills on the job, and (c) learning from colleagues or students. Representative comments from the qualitative survey data illustrate these themes.

- My own teacher preparation program did not prepare me for culturally relevant teaching, so what I have to share is from my personal experience and what I have learned "on the job."

- I attended a couple trainings put on by Aguilar-Valdez that were some good resources and starting points. That was helpful, but this is a topic that is not something I've been trained for otherwise.

- Working to keep up to date on new ideas; getting strategies, etc. from others; finding out new ideas from a student teacher; reading; working hard.

For the second round of focused thematic coding, the components of the CSST conceptual framework (curriculum, instruction, and relationships) along with the themes from the first round of coding served as the guide to assign codes and group the data. For 
example, I coded statements having to do with acquiring preparedness on the job or acquiring preparedness by learning from colleagues or students around curriculum, instruction, or relationships using codes such as "C-OTJ" or "R-LCS" during this second round. Finally, I examined and grouped the codes from the second round by similarities and differences. Specifically, I placed the codes into three groups, resources, strategies, or importance. In the next section, I start by presenting the qualitative findings based on the second round of coding with the relationships component because that was the highest ranked component in the conceptual framework in the quantitative data; then, I follow with curriculum and instruction.

Relationships. In their survey comments, the cooperating teacher participants' expressed feelings of preparedness for the component of relationships based on strategies; but, they also regularly noted the importance of relationships in a science classroom. For example, when asked to describe the thoughts about the ratings they gave themselves for the relationships section, one participant said, "This is of utmost importance to me and I believe these items have to be in place for learning to happen. These items are integral and are worth time, even away from curriculum content, to reinforce.” Participants also commented on specific strategies for the relationships component including getting to know each student, listening, making sure each student had access to the investigations. The comments about the relationships component focused on the ways that cooperating teachers acquired their preparedness through experience learning with and from students in the classroom. 
Curriculum. While also expressing feelings of preparedness, the participants' comments related to curriculum did not express the same level of importance; their comments focused on resources or strategies obtained through professional development. For example, when asked to describe their ratings for the curriculum section, one participant said, "I use a wonderful tool called a People's Curriculum for the Earth that I picked up at the Social Justice conference at Madison High School.”

Instruction. The comments shared for the instruction component were similar to those shared for the curriculum component, in that participants expressed feelings of preparedness mainly about resources or strategies obtained from professional development. For example, when asked to describe their ratings for the instruction section one participant said, "I have participated in activities with Okhee Lee who wrote the section of NGSS in favor of using phenomenon-based instruction so each learner can access the investigations." This comment in combination with the comment for the curriculum component illustrate the ways that the participants perceive their curriculum and instruction preparedness mainly in the context of professional development, where they learned and gathered resources and strategies from others. Whereas the comments for the relationships component while also focused on strategies were about strategies the participants had learned with and from colleagues and their students and included comments about the importance of these relationships in science teaching.

\section{Interpretation of Survey Qualitative Data}

In this section, I interpret the qualitative survey data to address the second subresearch question. The purpose of the qualitative data in this convergent mixed methods 
study was to validate and provide depth to the quantitative data. The sub-research question that I address in this interpretation is:

Research Question 1b: How do cooperating teachers describe their preparedness to support science teacher candidates to use the components of culturally sustaining science teaching? (Qualitative)

First, on a broad level the cooperating teacher participants' $(n=4)$ described that the preparedness they feel they have has come not from their own teacher preparation experience but rather what they have learned on the job, in professional development, or from their colleagues and students. When looking at descriptions of preparedness for each component, the participants described not only specific strategies they used to support teacher candidates to foster relationships of dignity and care with their students such as getting know each student, but also the importance of this aspect of their practice. The participants also described the ways in which they gathered resources or strategies that helped them support science teacher candidates to develop culturally mediated curriculum and facilitate learner-centered instruction such as using specific curriculum and attending professional development. The qualitative findings corroborate the quantitative findings that participants perceive themselves to be prepared in each component of the conceptual framework. Additionally, they corroborate the quantitative findings that while there may be no statistically significant differences in the degree to which they are expressing their perceived preparedness for each component of the CSST conceptual framework there are notable differences in the way participants are perceiving their preparedness and describing that preparedness. Along with corroborating the 
quantitative findings the qualitative survey findings add more depth in understanding the ways in which participants not only perceive and describe this preparedness but also feel they have become prepared. I explored these qualitative findings further in the focus group interview and discuss what came out of that in relation to the overarching research question in the next section with my interpretation of the findings overall.

\section{Interpretation of Findings}

As a final step in interpretation to address the overarching research question, I converged the quantitative and qualitative data and considered the focus group responses to strengthen the interpretation. The research question addressed in the rest of this section is:

Research Question 1: How do cooperating teachers perceive their preparedness to guide science teacher candidates to use the components (i.e., curriculum, instruction, relationships) of culturally sustaining science teaching?

The quantitative and qualitative survey data indicated that participants perceived themselves to be "prepared" to guide science teacher candidates to use each of the components (i.e., curriculum, instruction, relationships) of culturally sustaining science teaching. The participants perceived their preparedness around these components to similar degrees and described this preparedness based not on how they were prepared as teacher candidates but on what they have learned during their time teaching science. As the quantitative findings show, the mean scores for each component were in the "prepared" qualifier or between 4 and 5 on the survey Likert-scale $(M=4.86, M=4.15$, $M=4.10)$ with no statistically significant differences between the components $(p=0.916$, 
$p=0.074, p=0.074)$. While no statistically significant differences were shown between the components, the mean score ranks are important to consider, especially with the qualitative findings. The relationships component ranked the highest degree of preparedness $(M=4.68, U=4.00)$ followed by the curriculum component $(M=4.15, U=$ 12.00) and then the instruction component $(M=4.10, U=12.00)$, respectively.

The qualitative findings provided depth to the quantitative finding about how participants perceive their preparedness with respect to the CSST conceptual framework components. When describing their preparedness for each of the components, I noted a similar broad theme of cooperating teacher participants not feeling originally prepared in their own teacher preparation programs but feeling prepared now. The participants described that they have become prepared to support their science teacher candidates to use culturally sustaining practices through their teaching experience, professional development, and learning from their colleagues and students. These comments were especially apparent in the curriculum and instruction components where participants described acquiring strategies and resources through professional development. The participants who described qualitatively gathering resources and strategies from professional development to support their teacher candidates to develop culturally mediated curriculum and facilitate learner-centered instruction expressed some, though less, preparedness related to the relationships components where participants' comment differed the most. For the relationships component participants' described strategies they acquired and use to support teacher candidates as well as the importance around fostering relationships of dignity and care with their students to the practice of science teachers. 
These qualitative differences converged with the mean score ranks for the components illustrate the nuanced differences between how participants perceived their preparedness for the CSST components. Based on these converged results, is logical that there would be a higher degree of preparedness expressed for the relationships component shown in lower $p$-values for the Mann-Whitney tests comparing the relationships component to the curriculum and instruction component.

Focus group. During the focus group interview, the participants $(n=2)$ made a number of comments that gave more insight into these converged findings. To strengthen my interpretation of participants' perception of their preparedness to guide science teacher candidates to use the components (i.e., curriculum, instruction, relationships) of the CSST framework, I asked the focus group participants about the mean score ranks of the conceptual framework components. I also asked them about the themes developed from the qualitative coding relating to each of the components. Additionally, I afforded the focus group interview participants with an opportunity to speak into the differences and similarities in the constructs within the components evident in the descriptive statistics as slightly higher or lower than "prepared" qualifier on the survey. The focus group participants also had a chance to speak in general about their thoughts related to my initial analysis of the aggregate survey results.

When asked about the mean rank order of the components with relationships being the highest followed by curriculum and then instruction, both of the participants validated the survey results stating that they felt those results were accurate. The importance of science teachers, especially cooperating teachers, needing to establish 
relationships with both their students and their teacher candidates to be a trusted source of information came up multiple times and in multiple ways in the discussion.

Representative comments from the focus group interview illustrate this validation.

- I think it's accurate. I think that the relationships that exist in several regards, one between the cooperating teacher and the student teacher is incredibly important when it comes to the transfer of knowledge and making suggestions to assist pre-service teachers in being able to meet the needs of marginalized children better. Secondly, relationships are important because I think new teachers often don't understand the importance of relationships between themselves and their students or how to create those relationships...

- That trust relationship that we have as science teachers, especially now with NGSS when we talk about global warming or climate change and they hear so many different stories from politicians or perhaps their parents and classmates that when we provide information if we are not a trusted source we have to be very careful as science teachers in terms of not violating that trust...

- ...There are so many innate behaviors that make a good teacher a great teacher, and relationships are all wrapped up in that but I think it is really part of the cooperating teacher to help grow those pieces to identify them and grow them within that student teacher...

These particular comments validated and provided strength to my interpretation of the ways in which the participants expressed quantitatively that they felt "prepared" to support teacher candidates in fostering relationships of dignity and care with their 
students. Their comments about relationships corroborated the highest construct of 3.2 “develop and maintain positive, meaningful, caring, and trusting relationships with students" $\left(M_{3.2}=5.08\right)$. Their comments also validated and strengthened my interpretation of the way participants described qualitatively the importance of supporting science teacher candidates to develop these relationships with their students. Based on the convergence of the quantitative data and the qualitative data regarding the relationships component, participants perceive themselves to not only be "prepared" to support their science teacher candidates to use these approaches but also feel it is a crucial part of the work they do with their teacher candidates.

The focus group comments about curriculum and instruction provided further validation that participants felt "prepared" to support their teacher candidates with these components. While there were no statistically significant differences found, some differences do exist in the participants' perceived preparedness to support science teacher candidates as compared to the relationships component. As with the qualitative survey data, the commentary from the focus group interview for curriculum and instruction focused mainly on the strategies or resources acquired and used. Focus group participant comments aligned with the converged survey findings around the higher constructs: 1.4 "include a variety of instructional methods to match students' learning preferences, and maintain their attention and interest in science" $\left(M_{1.4}=4.83\right)$; and 2.3 "create a community of learners by encouraging students to focus on collective work, responsibility, and cooperation when learning science" $\left(M_{2.3}=4.75\right)$. Their comments included: 
- I think it's been part of the standards for a while and I think NGSS even makes it even more so that cooperative work, lab work, engineering, inquiry and all those things are necessary skills...Because I think fortunately the people who designed standards recognize that the workplace has changed from an individual achievement to a group achievement and a group work product and our students have to learn that group approach and I see that broadly in place in the school.

- He (a teacher candidate) was so fluent in the language not to mention playing soccer and everything that was meaningful to the young men that he taught in 8th grade immersion program. He spent a lot of time translating but it came so easily for him so we would put together these quick activities...My hope is that, I know these lessons are being scripted, but he takes it builds it and it's ready to go and that taught me a lot about how to really be a better mentor teacher.

These comments illustrated the ways in which cooperating teachers may be supporting their science teacher candidates to develop culturally mediated curriculum or facilitate student-centered instruction that helps to corroborate the quantitative and qualitative converged findings.

In the focus group, participants spoke of an additional aspect in relation to curriculum and instruction. This additional aspect was about both the systemic supports and barriers that affect their ability to support science teacher candidates to use the CSST approaches. These systemic supports and barriers came up often when discussing 
curriculum and instruction, particularly for the lowest construct: 2.2 "use a variety of linguistic styles with culturally diverse students in an attempt to communicate in culturally responsive or sustaining ways during science instruction" $\left(M_{2.2}=3.67\right)$. Focus group participants shared:

- Our teachers are probably hampered a bit by the instructional materials that is part of the curriculum. The freshman physics textbook is very Eurocentric and also very regionally specific culturally...As a cooperating teacher there needs to be an emphasis on supplementing that textbook language with culturally sensitive language and problems so that all students truly have access to the material.

- I think it's about bridging to whatever language the student is in the moment very quickly so that they can continue their thinking so that is what I would try to teach if I had another student teacher for sure.

Their comments considered in light of the converged quantitative and qualitative data helped to strengthen my understanding that participants perceive themselves to be "prepared" with respect to the curriculum and instruction components. However, their perception of their preparedness is not necessarily to the same degree or described in the same ways for each of the curriculum and instruction constructs.

While I did not ask the focus group participants directly about the nuances of the data from Mann-Whitney test for each component based on demographic factor, participants made a number of comments that inform the implications and potential next steps for research from these findings. Their comments were mainly in response to a 
question about element of 1.5 "design science curriculum that includes students obtaining, evaluating, and communicating information about systems of power, privilege, and marginalization" $\left(M_{1.5}=3.75\right)$, but also came up at various other points in the focus group interview. The participants spoke about the views cooperating teachers and teacher candidates may have of their traditionally underserved learners and the impact on their preparation to become a science teacher. The focus group participants shared:

- There is still a feeling, widely among science teachers, a deficit theory that marginalized students don't want to take harder courses but when you actually go out and survey classrooms it is different. I teach an ESL chemistry class and when I asked them how many of you would like to take an advanced science course in the next year or two every hand in the room will go up.

- It [curriculum that addresses power, privilege, and marginalization] doesn't get addressed a lot, do you see anything in the Next Generation science that gets at that? I see somethings that discourage the conversation...

Focus group participants also made comments related to the 1.5 construct that provided validation to the broad theme identified in the qualitative survey data about participants not feeling prepared for CSST in their teacher preparation programs. Participants stated:

- I think that typical MAT programs don't deal a whole lot in terms of general teacher preparedness with power privilege and marginalization... So, I think in general I see a lot of very good teachers but I just don't see that things such as power privilege and marginalization, if those are discussion points they are not particularly points of discussion within the curriculum. 
- When I think back to my MAT program there was very little direct training on how to work with marginalized children. I think that in there was in general an emphasis on social justice and equal opportunity but there wasn't much that translated into classroom instruction.

The participants' comments about power, privilege, and marginalization within CSST (particularly with respect to curriculum) along with the comments for relationships that addressed the NGSS illustrate the tension of the NGSS as a guide for these strengthsbased approaches. They also illuminate the complexities of teacher preparation programs adequately addressing these aspects of CSST within the context of the NGSS. The participants spoke about the standards being both helpful and hindering in their efforts to prepare science teacher candidates to support traditionally underserved students. Considering that the student demographic factor of having $50 \%$ or greater students of color in the most diverse class may be more influential for the curriculum and relationships component than the statistics indicated for the components overall. Comments from the focus group participants were especially poignant and highlight why further research in this area could be beneficial.

\section{Limitations of the Study}

While I used methods to support the validity and reliability of my findings, I need to address the limitations of my study. In this section, I explain these limitations and the ways in which they could affect my interpretation of the findings. As explained in Chapter 3, Creswell and Plano-Clark (2007) outlined a number of threats to validity and reliability that can arise during the data collection and analysis processes of a convergent 
mixed methods study. I consider these threats as well as claims from other scholars to discuss the limitations of my study.

One factor affecting the validity and reliability of mixed methods studies is sample size (Creswell \& Plano-Clark, 2007; Fowler, 2013). The total number of survey participants was only 12 cooperating teachers from the River School District completing the Culturally Sustaining Science Teaching Preparedness Survey—a modification of Hsiao’s (2015) Culturally Responsive Teacher Preparation Scale. Nevertheless, I claim the data from the survey has value for two main reasons. First, the total number of survey participants was $60 \%$ of the cooperating teachers in the River School District, which is a strong response rate and shows that the quantitative data represents the cooperating teacher population in the River School District. Second, I used a statistical test, the Mann-Whitney, intended for use with smaller sample sizes (less than 20) (Field, 2018). Thus, I contend that the small sample size of survey participants is not a major limitation of this study. However, the sample size of qualitative responses on the survey and the number of focus group interview participants were limitations of my study.

Creswell and Plano-Clark (2007) explained that threats to validity and reliability exist in convergent mixed methods studies when there are differences in the sample population or sample sizes between the quantitative and qualitative parts of the study and this is where I see the main limitation of my study. The sample population remained consistent, but the sample sizes differed. While I attempted to mitigate the threats of differing sample sizes in the design of the study by including all the quantitative and qualitative aspects of data collection in one survey, the sample size of quantitative data 
differed from the sample size of the qualitative data. Most of the quantitative data represents 12 participants with the exception of the demographic factors data that represents 11 of 12 participants. In contrast, the qualitative survey data set represents only four survey participants, because only four participants provided substantive responses to analyze. Another limitation related to sample size was the number of focus group participants. Due to difficulties recruiting cooperating teacher participants for the focus group interview, there were only two participants. Thus, a main limitation of my study is that the qualitative survey data and focus group interview only represent some of the voices of the survey participants.

With only four participants providing substantive qualitative data on the survey, I had less data than if all the participants had completed the qualitative sections of the survey. With the limitation of less qualitative survey data, important voices and views could have been missing in the analysis process, potentially leading to bias in my analysis (Creswell \& Plano-Clark, 2007). If more participants had shared substantive comments on the survey, I could have identified alternative or additional themes during the two rounds of coding, especially during the holistic coding round of the analysis. For the focus group interview, a smaller sample size did not lead to less data as the focus group participants had a great deal to say and the interview lasted a full hour adding depth to the information I had available to strengthen my overall interpretation of the results. Yet, the small size of the focus group did result in limited points of view shared about my initial interpretation of the survey data. Had more people been present in the focus group interview, I may have heard countering views not shared by the two participants of the 
focus group. I expand upon the ways the introduction of bias was a limitation in this study later in this section.

While I see the smaller sample size of the qualitative survey data and the focus group interview as the main limitations of the study, I think the data from this study remain valid and reliable for a number of reasons. First, the cooperating teachers who did provide the qualitative survey data and participated in the focus group interview represented a range of degrees of expressed preparedness on the survey (see Table 7). Their preparedness included (a) one participant with the highest mean score $\left(M_{\mathrm{B}}=6.0\right)$ or within the "highly prepared" qualifier; (b) two participants with mean scores falling within the "prepared" qualifier $\left(M_{\mathrm{A}}=4.87, M_{\mathrm{G}}=4.0\right)$; and (c) two participants falling within the "somewhat prepared" qualifier $\left(M_{\mathrm{F}}=3.73, M_{\mathrm{H}}=3.73\right)$. These participants not only represented a range of perceived preparedness, but also varied within the demographic factors such as grade level taught, years as a cooperating teacher, and student demographics of their most diverse class (see Tables 3 and 4). Second, I designed this study to emphasize the quantitative data with the qualitative data serving as a way to validate and corroborate that quantitative data. Thus, the lower number of qualitative survey responses and lower amount of qualitative data, while certainly a limitation, provide a reliable source to use for the purposes of validation and corroboration, especially because it came from participants representing a range of degrees of preparedness expressed on the quantitative data and varying demographic factors. Third, the purpose of the focus group interview was not to introduce an entirely new data source for analysis; the purpose was to strengthen my interpretation of the 
survey data sources through member checking (Merriam \& Tisdell, 2016). Thus, again, while the lower number of participants is certainly a limitation, it remains a reliable source of data to use for the purposes of strengthening my interpretation of the survey data.

In addition to the limitation of small sample sizes from the survey qualitative data and focus group interview participants, there is always the threat of introducing bias into a study, whether quantitative, qualitative or mixed methods. The limitations around bias in this study - along with the limitation of bias that could have been introduced by the participants with smaller sample sizes - were biases that I may have introduced into the process as the researcher. As the researcher, I was the sole coder for the qualitative survey data, and I conducted the focus group interview. As the sole coder for the qualitative survey data, it is possible that I may have been seeing only what I wanted to see (Merriam \& Tisdell, 2016), resulting in a biased interpretation of the findings. To address this limitation, I conducted multiple rounds of coding, each with a different focus. I also brought my initial analysis of the qualitative survey data to the focus group participants for them to speak into the ways I was seeing the data and have a chance to say if they saw it any differently. As the focus group interviewer, I may have introduced bias in the questions that I asked or the way I asked them (Morgan, 1996). To attempt to address this limitation, I designed the questions to be strictly about providing feedback on my preliminary analysis of the survey data. I included questions about aspects of the quantitative data and qualitative data analysis and I included questions about both the strengths and needs that I identified during the preliminary analysis. I also provided the 
participants a copy of the questions when they arrived at the interview so that they could both listen to and read the questions as I asked them.

Finally, another limitation of this study was the fact that the survey is self-report, and the participants may have reported socially desirable responses (Creswell, 2014), meaning that my findings could be skewed more toward strengths than they would have been had additional data collection methods been used. For example, self-report methods alone did not allow me to observe the levels of preparedness reported by cooperating teacher participants in action to determine if their practices reflected their reported feelings of preparedness. Yet, individual data from the survey, both quantitative and qualitative, illustrated that a number of the participants indicated areas in which they did not feel prepared, showing that participants did not only express or describe socially desirable responses. In addition, other recent self-report studies conducted with cooperating teachers did not yield positive results from the participants (ThomasAlexander \& Harper, 2017), which I assert may be less of a limitation. Even if I had conducted observations, my results could have been skewed toward more strengths because my presence as an observer could influence the participants' practices during the observation as compared to their typical practice (Merriam \& Tisdell, 2014). Nevertheless, I addressed the self-report limitations in that I situated this study within the pragmatic paradigm, signifying that I was looking for the participants socially constructed meaning of their own preparedness. Understanding the cooperating teacher participants socially constructed meaning of their preparedness is important because perceptions influence self-efficacy, which in turn, influence practice (Bandura, 2002; 
Matsko et al., 2018). I claim that understanding cooperating teacher perceived preparedness is a crucial first step in understanding their preparedness in practice and honoring the important work these professionals do in preparing science teacher candidates. Thus, despite the limitations present in this study, I claim that the findings from this study are worth considering in the larger context of the problem of practice guiding this study and could inform the work of science education practitioners and researchers.

In this chapter, I shared the findings from my data collection and analysis. I analyzed and presented the demographic information from the participants. I also analyzed and interpreted the quantitative and qualitative survey results to address Research Question 1a and Research Question 1b. Finally, I converged the two data sets and considered them in light of the focus group interview to strengthen my overall interpretation of the results to address the overarching Research Question 1. In Chapter 5, I synthesize the findings, situate them in the larger context of the problem and literature, and outline the ways I think the findings from this study can be interpreted to have implications for practice, as well as next steps for research. 


\section{Chapter 5: Conclusion}

The problem of practice guiding this study was that within the shifting landscape of STEM education, too few science teachers are prepared to implement the new standards in ways that are culturally sustaining for their traditionally underserved learners. The purpose of this mixed methods study was to describe teacher educators'specifically cooperating teachers-perceived preparedness to support science teacher candidates to use culturally sustaining pedagogies. My review of the literature indicated that teachers and teacher educators in general (Hawkins, 2016; Johnson, 2011; Marshall \& Smart, 2013; Moseley et al., 2014; Sleeter, 2017; Underwood \& Mensah, 2018) and mentor or cooperating teachers in particular (Thomas-Alexander \& Harper, 2017; Vass, 2017) — despite the major role they play in the development of teacher candidates (Clarke et al., 2014; Matsko et al., 2018; Ronfeldt et al., 2018) are not equipped to prepare teacher candidates for culturally relevant pedagogies (Ladson-Billings, 1995) (CRP), culturally responsive teaching (Gay, 2010) (CResP), or culturally sustaining pedagogies (Paris \& Alim, 2017) (CSP). Specifically, current literature on cooperating or mentor teacher's preparedness with respect to CSP, while limited, has revealed some alarming results as to the mindsets, efficacies, and roles of these professionals in supporting science teacher candidates to use such pedagogies. While an emerging body of literature examines teacher preparation for CSP in general, there is a paucity of research around preparing science teacher candidates for CSP. Specifically, little research exists related to preparing science teacher candidates within the context of the NGSS and the role of teacher educators such as cooperating teachers. In my study, I intended to fill some gaps 
in the literature and to add to the knowledge base regarding science teacher candidate's preparation. To this end, I addressed the following research questions:

Research Question 1: How do cooperating teachers perceive their preparedness to guide science teacher candidates to use the components (i.e., curriculum, instruction, relationships) of culturally sustaining science teaching?

Research Question 1a: To what degree do cooperating teachers feel prepared to support science teacher candidates to use the components of culturally sustaining science teaching? (Quantitative)

Research Question 1b: How do cooperating teachers describe their preparedness to support science teacher candidates to use the components of culturally sustaining science teaching? (Qualitative)

In this chapter, I synthesize the findings related to these research questions within the larger context of the problem of practice, purpose of the study, and literature (both theoretical and empirical). My study was a convergent mixed methods study so I focus the synthesis of the findings on the overarching research question (Research Question 1), which I addressed through convergence of the findings from the other two sub-research questions. Then, I use the synthesis of the findings to outline implications for practice including how the findings can inform the design and development of (a) learning experiences for cooperating teachers who host science teacher candidates, (b) strategic placements and experiences for science teacher candidates with their cooperating teachers, and (c) policies that highlight those cooperating teachers who work with science teacher candidates during the clinical practice experience. I conclude by suggesting next 
steps of research some of which I see as my next steps in my professional agenda as a scholar.

\section{Synthesis of Findings}

I situate the findings of my study within the larger context of the landscape of STEM education that has shifted in significant ways that affect the preparation of science teacher candidates. In my research, I focused on two of those shifts: (a) new national science standards, the Next Generation Science Standards (NGSS), which emphasize inquiry-based instructional methods through specific science and engineering (S\&E) practices; and (b) an increasingly diverse student population that can benefit from pedagogies that are culturally sustaining. The NGSS, adopted in many states across the United States, including Oregon (National Research Council, 2012), intended to broaden the view of what science is, how people do science, and who does science (Januszyk et al., 2016). The NGSS shifted "science educators' focus from simply teaching science ideas to helping students figure out phenomena and design solutions to problems" (Krajcik, 2015, p. 6). Thus, the standards also shifted the landscape of STEM education toward an inquiry-based learning approach (Bybee et al., 2006) with an emphasis on S\&E practices (Brown, 2017). Many scholars claim, and I agree, that despite the NGSS being based on years of research for teaching science to all students (Windshitl \& Stroupe, 2017), preparing science teachers for the standards alone is not enough to equip them to serve their traditionally underserved learners in increasingly diverse student populations (Brown, 2017; Meyer \& Crawford, 2011; Rodriguez, 2015). To explore the effects of these two major shifts on the preparation of science teacher candidates, I grounded this 
study in the theoretical framework of critical race theory (Ladson-Billings, 2009) and social constructivism theory (Vygotsky, 1978). Within this theoretical framework, I base my findings on the conceptual framework outlined from the theoretical and empirical literature. Broadly, I grounded the conceptual framework in critical race theory and Gay's (2010) tenets of culturally responsive teaching practices. I also grounded my research in some of the culturally sustaining pedagogy concepts put forth by Paris and Alim (2017) that built upon Ladson-Billings (1995) foundational culturally relevant pedagogy work. I also based the conceptual framework on Brown (2017) and Dodo Seriki's (2018) positions that certain attributes of culturally relevant and culturally responsive science practices are complementary to certain inquiry-based instruction methods, including the NGSS S\&E practices. Thus, my conceptual framework built not only on the works of Ladson-Billings (1995), Gay (2010), and Paris and Alim (2017) around culturally relevant, responsive, and sustaining pedagogies through critical race theory, but also on social constructivism theory with the inquiry-based science practices suggested to be complementary to attributes of culturally responsive science practices (Brown, 2017; Dodo Seriki, 2018). The following list represents a culturally sustaining science teaching (CSST) conceptual framework (see Figure 2 for a visual representation).

- The science teacher candidate is prepared to develop culturally mediated curriculum that includes students' cultural identities (Gay, 2010) and real world connections to students lived experiences including students obtaining, evaluating, and communicating information (Brown, 2017) about systems of power and oppression in science (Paris \& Alim, 2017). 
- The science teacher candidate is prepared to facilitate learner-centered instruction that promotes agency and input from all students (Gay, 2010) and centers on collective and dynamic community languages as assets (Paris \& Alim, 2017) to learning as students develop and use models that represent a broader understanding of science concepts (Brown, 2017).

- The science teacher candidate is prepared to foster relationships of dignity and care (Paris \& Alim, 2017) grounded in positive perceptions that communicate high expectations to all students within a collaborative learning community (Gay, 2010) where students work together to construct explanations and designing solutions to problems or challenges (Brown, 2017).

Addressing the overarching research question. How do cooperating teachers perceive their preparedness to guide science teacher candidates to use the components (i.e., curriculum, instruction, relationships) of culturally sustaining science teaching? From the converged quantitative and qualitative findings, the cooperating teachers from River School District who participated in this study perceived themselves to be "prepared" to support science teacher candidates with each of the components of the CSST conceptual framework. The cooperating teacher participants perceived their preparedness not in the context of what they learned during their own teacher preparation program, but rather from what they learned on the job as teachers and cooperating teachers. Findings from the Culturally Sustaining Science Teaching Preparedness Survey (adapted from Hsiao, 2015) about cooperating teacher participants feelings of acquired preparedness for the components of CSST were corroborated and strengthened by the 
focus group interview comments. The focus group participants corroborated the findings that these cooperating teacher participants felt their preparedness was acquired on the job rather than from their teacher preparation programs.

The findings that the cooperating teacher participants perceived themselves to be "prepared," though not from their own teacher preparation programs, align with scholars' claims about the challenges of preparing teacher candidates to use CRP, CResP, and CSP within the current educational context (Dominguez, 2017; Sleeter, 2017; ThomasAlexander \& Harper, 2017; Underwood \& Mensah, 2018; Vass, 2017). When examined through the theoretical framework of critical race theory and social constructivism, the findings of my study become even more apparent in the context of the problem of practice. The problem of practice is that within the shifting landscape of STEM education too few science teachers are prepared to implement the new standards in ways that are culturally sustaining for their traditionally underserved learners. Despite diversification of classrooms, the teaching and teacher educator workforce has remained predominantly White (Dominguez, 2017). The dominant teacher force can contribute to the perpetuation of practices that favor dominant cultural approaches to knowing and doing science (Civil, 2016; Delgado Bernal \& Villapando, 2016; Meyer \& Crawford, 2011; Rodriguez, 2015) and exacerbate the racial achievement gap and deficit mindsets about students of color abilities (Sleeter, 2017; Taylor et al., 2009). Teacher candidates in teacher preparation programs (Smith-Maddox \& Solórzano, 2002) and all teacher educators, including cooperating teachers (Thomas-Alexander \& Harper, 2017; Underwood \& Mensah, 2018; Vass, 2017) need more opportunities to understand and use 
strengths-based approaches such CRP, CResP, and CSP in the sociocultural context of diversifying school classrooms. Even with the challenges outlined by scholars contributing to my theoretical and conceptual framework, my findings are encouraging. In spite of not feeling that they acquired preparedness around CSST in their teacher preparation programs, the cooperating teacher participants expressed that because of what they have learned on the job - through professional development, and collaboration with colleagues and students - that they felt "prepared" to support their science teacher candidates to use these approaches. In other words, although the generation of science teacher candidates who are now serving as cooperating teachers did not perceive that they were prepared with these strengths-based approaches, they now feel prepared to support the next generation of science teacher candidates to use such approaches in their future classrooms.

While I found no statistically significant differences between the components of my conceptual framework, quantitative and qualitative analyses helped me to address the research question with more depth. The quantitative differences were evident in the mean rank orders from the Mann-Whitney tests with the relationships component $(M=$ 4.68, $U=4.00$ ) greater than the means of both the curriculum and instruction components $(M=4.15, U=12.00)$ and $(M=4.10, U=12.00)$, respectively. The qualitative differences appeared in the survey comments and focus group interview. The cooperating teacher participants made comments for all components about strategies that they used to support teacher candidates with these approaches but for the relationships component they also made comments about the importance of supporting teacher 
candidates with these approaches. Thus, while not statistically different, cooperating teacher participants perceived their preparedness for the relationships component with some differences as compared to the curriculum and instruction components that were similar in perceived preparedness.

\section{Situated in the Larger Context}

According to Braaten and Sheth (2016), science teachers trying to teach science for equity face tensions that arise from the political context (i.e., standards) (Fowler, 2013) about what counts as valid science knowledge and practice. Rodriguez (2015) asserted that dominant cultural approaches to knowing and doing science within the scientific community heavily influenced the development of the NGSS. Tan and Barton (2010) claimed, as other scholars had (Aikenhead \& Jedege, 1999; Costa, 1995), that helping students create a bridge between their funds of knowledge (i.e., assets) and the scientific understandings espoused in school standards, such as the NGSS, was a crucial part of engaging in anti-oppressive science teaching. Laughter and Adam's (2012) suggested that when working to facilitate instruction that provides students opportunities to bridge their own culture and the scientific understandings taught in school that it is most beneficial to incorporate culturally relevant or responsive approaches throughout the curriculum. In studying the effects of such alternative curriculum, Kanter and Konstantopoulos (2010) contended that inquiry-based instruction if further pursued within the framework of CRP could support the development of curricula that advances more equitable science education. When considering the findings of the nuanced differences between the components of the conceptual framework in my study, a number 
of scholars' (Braaten \& Sheth, 2016; Rodriguez, 2015; Tan \& Barton, 2010) findings indicate that differences and similarities within the conceptual framework components could exist. Particularly, it is logical that the relationships component, the component least influenced by the standards and most necessary in incorporating student's funds of knowledge into curriculum and instruction, would be perceived by the cooperating teacher participants with some differences. It is reasonable within the context of the literature that the teachers' perceived the relationships component with a higher degree of preparedness and be described with an emphasis on not only strategies but also the importance of this to their practice. Additionally, the literature illustrates the connectedness between the curriculum and instruction components of the conceptual framework (Brown \& Crippen, 2016a; Kanter \& Konstantopoulos; 2010; Laughter \& Adams, 2012), so it is logical that these two components would be similar with regard to cooperating teacher participants' perceived preparedness.

The findings of the nuanced differences in perceived preparedness between the components of the conceptual framework, in light of the literature about the tensions within the political context of education, were evident in the focus group interview commentary. A number of the comments from the focus group illustrated the tension science teachers face using the NGSS as a guide for CSST. The NGSS standards appear to help in some ways and hinder in other ways. Given Haag and Megowan's (2015) study that revealed many classroom teachers across the United States (some of whom are serving as cooperating teachers) do not feel prepared for the NGSS, the tensions apparent in these comments about the NGSS were not surprising. Yet, even with the tensions from 
these comments about the NGSS, the participants in my study still expressed feelings of preparedness to support their science teacher candidates with the components of CSST in the current educational context.

The nuanced differences between these components also showed up in other ways in the quantitative and qualitative data. First, the constructs of the components differed (see Table 6) in that the constructs in the relationships component were all at the "prepared" qualifier or in one instance even at the "highly prepared" qualifier $\left(M_{3.1}=\right.$ $\left.4.58, M_{3.2}=5.08, M_{3.3}=4.67, M_{3.4}=4.5, M_{3.5}=4.58\right)$. Whereas the curriculum and instruction components while containing the second and third highest constructs overall $\left(M_{1.4}=4.83, M_{2.3}=4.75\right)$ also contained a number of constructs at the "somewhat prepared" qualifier $\left(M_{1.2}=3.92, M_{1.3}=3.92, M_{1.5}=3.75, M_{2.2}=3.67, M_{2.5}=3.92\right)$. The construct results in my study are consistent with much of the literature on CRP and CResP. Within the context of the literature, construct 3.2 "develop and maintain positive, meaningful, caring, and trusting relationships with students," and construct 1.4 “include a variety of instructional methods to match students' learning preferences, and maintain their attention and interest in science" would reasonably be among the highest constructs in the conceptual framework. Scholars found that relationships and curriculum — ones including trust, high expectations, and student strengths — are the foundation upon which CRP, CResP, and CSP are built in practice (Aikenhead \& Jedege, 1999; Bettez et al., 2011; Brown, 2017; Costa, 1995; Dominguez, 2017; Gay, 2010; Johnson, 2011; Ladson-Billings, 1995; Luft et al., 1998; Lund \& Lee, 2015; Paris, 2016; Tan \& Barton, 2010). Whereas, the constructs of 2.2 "use a variety of linguistic styles 
with culturally diverse students in an attempt to communicate in culturally responsive or sustaining ways during science instruction;" and 1.5 "design science curriculum that includes students obtaining, evaluating, and communicating information about systems of power, privilege, and marginalization" would plausibly be among the lowest. Other scholars claimed that discourse and sociopolitical consciousness are not as apparent in curriculum and instruction as other attributes of these frameworks (Brown, 2017; Brown \& Crippen, 2016b; Johnson, 2011; Powell et al. 2012).

Additionally, when looking at the demographic factors, the results of the MannWhitney tests for the student demographic factor of $50 \%$ or greater students of color in the most diverse class were quite different for the relationships $(p=0.172)$ and curriculum $(p=0.179)$ components than for the instruction $(p=0.918)$ component. While I did not explore these factors directly in the focus group interview, I found a number of comments that appear consistent with the literature- that even with good intention and efforts, deficit mindsets about students of color still plague today's science classrooms (Delago-Bernal \& Villepando, 2016). When considering my findings through the theoretical framework about what and whose knowledge is legitimate in the sociocultural cultural context of the science classroom and science teacher preparation, I noticed areas worth exploring further in future research. I discuss these in the next section of this chapter.

Contradictions to literature. While a number of the findings of my study are either consistent with or logical in light of what other scholars have found or claimed in the literature, the results from my study also illustrate findings that differ from the recent 
literature. For example, the finding that cooperating teacher participants perceive themselves to be "prepared" to support science teacher candidates with the components of CSST seems to contradict two studies in recent literature that illuminated some alarming results about cooperating teachers' mindsets, efficacies and roles in working with science teacher candidates to understand and use CSP. First, Thomas-Alexander and Harper (2017) found in their mixed methods study that included elements of culturally responsive teaching efficacy as well as responses to open-ended prompts that the mentor or cooperating teachers "expressed overwhelmingly negative views of the students, school, and communities" (p. 49). Parallel to and supportive of the findings of Thomas-Alexander and Harper (2017) were those of Vass (2017) who found that teacher candidates working toward culturally responsive schooling faced barriers from their mentor teachers. The barriers Vass (2017) identified included, "mentors encouraging limited and limiting curricula, pedagogic and assessment practices; mentors communicating resistance to doing things differently or valuing cultural responsiveness; and a fearful awareness of being evaluated by their mentors" (p. 451). The findings from my study are also in contrast to the findings of Thomas-Alexander and Harper (2017) and Vass (2017). In my study, the cooperating teacher participants expressed preparedness around CSST and described the strategies they used with candidates to develop these approaches as well as the importance of these approaches. The conflicting findings of my study and those findings of Thomas-Alexander and Harper (2017) and Vass (2017) are encouraging, particularly in the context of the problem of practice. 
I did frame my research in light of cooperating teacher's strengths, and I acknowledge the limitations of my study (as described in Chapter 4). These limitations make it difficult to compare the results directly with the results of other studies. Nevertheless, my findings are encouraging as another lens to examine the preparedness of cooperating teachers, one focused on the ways in which they may support their teacher candidates rather than hinder them. With the encouraging findings from my study and a strengths-based approach to the interpretation in mind, I discuss the implications of this study in the next section.

\section{Implications}

Due to the small sample size, I do not suggest that the results of my research are generalizable; however, I assert the importance of the results. Although the results rely on self-report methods of participants' perceptions, these results have implications for practice. The perspectives of cooperating teachers in teacher preparation (Clarke et al., 2014; Matsko et al., 2018; Ronfeldt et al., 2018; White \& Forgasz, 2016) and their feelings of preparedness have an influence on their practice (Bandura, 2002; Matsko et al., 2018). In the context of my study, one aimed at describing teacher educators'specifically cooperating teachers - perceived preparedness to support science teacher candidates to use CSP to inform practices and policies that influence STEM teacher preparation the findings are valuable. I note three main areas where findings from my study have implications for practice and policies regarding the preparation of science teacher candidates. These implications include (a) learning experiences for cooperating teachers who host science teacher candidates, (b) strategic placements and experiences 
for science teacher candidates with their cooperating teachers, and (c) policies that highlight those cooperating teachers who work with science teacher candidates during the clinical practice experience. In the next section, I present the implications from my study for each of these areas and describe what I see as next steps for research.

Learning experience for cooperating teachers. The quantitative and qualitative date revealed that the cooperating teacher participants in River School District perceive themselves to be "prepared" to support science teacher candidates to develop culturally mediated curriculum, facilitate student-centered instruction, and foster relationships of dignity and care. While nuanced differences exist for the components and constructs within the CSST conceptual framework as to the degrees of preparedness and the descriptions around that preparedness, the findings illuminated that as a whole (with $60 \%$ surveyed) the cooperating teachers in River School District have many strengths to offer teacher candidates. The cooperating teacher participants in my study described their preparedness related to what they had learned in professional development and from their colleagues and students. Thus, I argue that the River School District could utilize the perceived strengths of these cooperating teachers to develop learning experiences for all cooperating teachers in the area.

For example, research has shown that professional development on learnercentered instruction has the potential to serve as a pathway for teachers to use more CRP or CResP (Brown \& Crippen, 2016a; Dole et al., 2015). The findings of my study along with those of Brown and Crippen (2016a) and Dole et al. (2015) illustrate how current science cooperating teachers could use learner-centered approaches to shift toward 
adopting CSP and could support the teacher candidates and their colleagues to do the same. As a caveat to my suggestion, the findings of my study indicate that while cooperating teacher participants perceived "preparedness" around the components of the CSST conceptual framework, there are nuanced differences in their preparedness for each of the constructs within the components of the framework as well as differences in their individual feelings of preparedness. Hence, it will be important to dig into those nuances when having cooperating teachers determine the focus area for professional development they would develop and deliver to their colleagues.

Additionally, as part of my study, I purposefully included cooperating teachers as teacher educators more holistically (Clarke et. al., 2014; Swennen at al., 2010). Based on the perceived preparedness demonstrated by the cooperating teachers in my study and what Underwood and Mensah (2018) found in their study of university-based teacher educators lack of understanding of CRP in practice, these learning experiences could extend to include other teacher educators. Involving other teacher educators, such as university professors or supervisors, in the focused learning experiences that cooperating teachers design and deliver could enhance teacher educators' abilities to support science teacher candidates to use these CSST approaches.

Strategic placements and experiences for science teacher candidates. The findings from my study could be useful not only for school districts, but also for university teacher preparation programs. In my study, the results revealed that the cooperating teachers perceived themselves to be "prepared" with the components of the conceptual framework, with a particular emphasis on the relationships component. 
Relationships - between the cooperating teacher and the teacher candidates as well as between the teacher candidates and their students - are a crucial part of the clinical practice experience for everyone. I recommend that university teacher preparation programs use the survey instrument—Culturally Sustaining Science Teaching Preparedness Survey (adapted from Hsiao, 2015) — to place teacher candidates strategically in their clinical practicum experiences. When looking at the individual mean scores in combination with qualitative survey and focus group interview comments, I note that some participants express more preparedness than others do. Thus, the results of my study and the Culturally Sustaining Science Teaching Preparedness Survey (adapted from Hsiao, 2015) instrument could inform university's about individual cooperating teachers who perceive themselves to be more or less prepared to support teacher candidates to develop within the CSST framework.

While the Culturally Sustaining Science Teaching Preparedness Survey (adapted from Hsiao, 2015) is self-report measure of perceived preparedness, I contend that it could identify cooperating teachers who may be strong fits as mentors. Identifying these cooperating teachers who can model the desired approaches (Darling-Hammond, 2006; Matsko et al., 2018) for teacher candidates who are making the transition from science student to science teacher (Kang et al., 2013) in the classroom with diverse student populations (Villegas \& Lucas, 2002) could enhance the teacher candidates own preparedness with such approaches (Ferber \& Nillas, 2010; Kissau et al., 2017). Additionally, I suggest that teacher education programs modify the Culturally Sustaining Science Teaching Preparedness Survey (adapted from Hsiao, 2015) to include open- 
ended prompts such as "Please describe strategies that you use to support teacher candidates with the approaches outlined in this section." For the purposes of my study, I intentionally designed the prompts to be open-ended, so I could see where the cooperating teachers went with their responses (Thomas-Alexander \& Harper, 2017) but if used for the purposes of informing strategic teacher candidate placement it could be more useful to tap into the specific strategies cooperating teachers are describing.

I also recommend, based on the promising preparedness findings of my study, using the strategic placement of teacher candidates with cooperating teachers in combination with tools that can enhance the teacher candidate abilities to learn from what is being modeled for them by the cooperating teacher. For example, Brown and Crippen (2016b) found that when teacher candidates used a specific protocol called the Growing Awareness Inventory during their observations of cooperating teachers delivering instruction, it helped to scaffold the teacher candidate's awareness of culturally responsive science practices. According to Brown and Crippen (2016b), teacher candidates developed their awareness of culturally responsive practices when they perceived their cooperating teachers as having these strengths. Culturally responsive practices included facilitating a respectful and collaborative classroom environment, and contextualizing instruction in the students' interests and experience. The culturally responsive practices developed in Brown and Crippen's (2016b) study aligns to the component and construct strengths identified in my study. The combination of strategic placement with a particular cooperating teacher based on their perceived preparedness with the strategic use of observation tools such as the Growing Awareness Inventory has 
the potential to enrich the learning of the science teacher candidate during their clinical practice experience.

Another lens through which teacher candidates in strategic placements with cooperating teachers could potentially benefit would be the lens of cultural humility. The participants in my study described their preparedness partly as a result of what they learned from and with their students, which aligns well with the concepts of cultural humility (Foronda et al., 2016). Tinkler and Tinkler (2016) found that themes around relationships with diverse students emerged when teacher candidates worked through a cultural humility lens. Given that relationships as a component of the CSST conceptual framework and all the constructs within it were strengths of the cooperating teachers participants in my study, there is great potential for university teacher preparation programs to design placement experiences that support the development of cultural humility. The development of cultural humility during teacher preparation could aid in promoting more culturally sustaining instructional practices in science classrooms (Tinkler \& Tinkler, 2016).

Policies that highlight cooperating teachers. Preparing teachers for methods espoused in policies such as standards that are markedly different from what they experienced either as learners in K-12 school or in their teacher preparation program requires explicit modeling (Bransford et al., 2000; Darling-Hammond, 2006; Lortie, 1975). The findings from my study suggest that participants - cooperating teachers within River School District — feel "prepared" to do such modeling. The implications for practice, which I described, require more time and dedicated effort from cooperating 
teachers who are already, in many cases, working beyond their compensation as cooperating teachers. Thus, I suggest, based on the strengths of participants illuminated in this study, that the River School District and its university partners, work together to develop policies to provide improved compensation for these professionals. These professionals deserve adequate compensation for the leadership role they are taking on in preparing the next generation of science teachers.

I contend that with improved compensation there is an opportunity to support cooperating teachers to continue to develop their strengths through professional development opportunities. Cooperating teacher participants in my study described their preparedness based on what they learned from professional development. The benefits of such professional development for promoting espoused practices are evident in the literature (Brown \& Crippen, 2016a; Dole et al., 2015). Thus, districts and universities need to consider professional development and training opportunities as they seek to compensate cooperating teachers better for the additional work they do in preparing teacher candidates. For example, cooperating teachers could (a) be paid a higher stipend when they develop and deliver professional development for their colleagues; (b) have time in their workday to dedicate to their cooperating teacher responsibilities such as training teacher candidates in their building or district on particular methods; or (c) have funds allocated to attend outside district professional conferences. Whatever the compensation, it needs to honor the extra work that cooperating teachers do with teacher candidates as well as propel that work forward. 
Additionally, in many cases, the only current requirement to be a cooperating teacher is years of experience and a willingness to serve in the role. According to Matsko et al. (2018), many of the requirements used to recruit cooperating teachers are not linked to either teacher candidate or student outcomes. Thus, I recommend the nomination of science cooperating teachers in the River School District. Specifically, I recommend that this nomination come not just from school administration, but in various forms. Nominations could come from sources including but not limited to self-nomination, nomination from or for a colleague (e.g., peer teacher, teacher on special assignment, instructional coach), nomination from a past teacher candidate, nomination from students, nomination from families in the community, or nomination from administrators. Having a range of agents in the teacher preparation network nominate cooperating teachers (Marion \& Gonzalez, 2014) could allow more perspectives to influence what counts as "quality" science teaching (Dominguez, 2017; Rodriguez, 2015), consequently influencing the preparation of science teacher candidates. I claim that nominations of and improved compensation for cooperating teachers could honor and propel the work of cooperating teachers who mentor science teacher candidates.

\section{Next Steps for Research}

While the results of my study revealed no statistically significant differences between the components of the CSST conceptual framework overall or when examined for each demographic factor, I recommend a closer look into patterns in the data. Particularly, I think it would be important to look into the student demographic factor of $50 \%$ or greater students of color in the most diverse class and how that impacted 
cooperating teacher perceived preparedness for each component of the conceptual framework. To examine this demographic factor further, I envision the design of a follow up study - a quantitative study that is a scaled up version of the Culturally Sustaining Science Teaching Preparedness Survey (adapted from Hsiao, 2015). Teacher preparation programs could survey cooperating teachers across the state of Oregon and use the quantitative Likert-scale items. While the focus would be on the student demographic factor, I recommend the inclusion of all the demographic factors of the survey included in my study because when scaled to a larger sample size, demographic factors could reveal new and interesting results worth examining.

Because this study did not look at the cooperating teacher interactions with their teacher candidates and only described their self-reported perceived preparedness, a next step in research could be to follow up with these cooperating teachers and find the ways in which their preparedness appears in the classroom with their teacher candidates. Specifically, I think it could be valuable to explore the specific strategies in action that cooperating teachers use to support their teacher candidates with CSST approaches. I also suggest a follow up case study of one or two cooperating teachers over their year placement with a teacher candidate. The case study could involve an examination of data collected from classroom observations of instruction and debriefing experiences, instructional and debriefing materials, and individual interviews with the cooperating teachers and the teacher candidates. By going beyond perceptions into classroom practice, a case study approach may help develop a deeper understanding of cooperating teacher preparedness to support teacher candidates with CSST. 
In this chapter, I synthesized the findings of this study within the context of the problem of practice, purpose of the study, and literature (both theoretical and empirical). I outlined implications for practice including how the findings of this study can inform the design and development of (a) learning experiences for cooperating teachers who host science teacher candidates, (b) strategic placements and experiences for science teacher candidates with their cooperating teachers, and (c) policies that highlight those cooperating teachers who work with science teacher candidates during the clinical practice experience. Finally, I suggested next steps for research, some of which I see as my next steps in my professional agenda as a scholar.

\section{Summary}

The findings of my study are not only encouraging as they offer a strengths-based perspective on cooperating teacher preparedness, but they are also particularly encouraging in the context of the problem of practice of my study. The problem of practice guiding this study was that within the shifting landscape of STEM education too few science teachers are prepared to implement the new standards in ways that are culturally sustaining for their traditionally underserved learners. Because I focused this study on cooperating teachers as teacher educators that influence science teacher candidates during their clinical practice experience (Clarke et al., 2014; Matsko et al., 2018; Ronfeldt et al., 2018; White \& Forgasz, 2016), I explored the following three critical connection points:

1. Culturally sustaining pedagogies build upon the foundations of culturally relevant pedagogies and culturally responsive teaching, but are not 
interchangeable concepts (Gay, 2010; Ladson-Billings, 1995; Paris \& Alim, 2017).

2. Culturally relevant or responsive and inquiry-based science instructions are complementary in some practices and attributes (Brown, 2017; Dodo Seriki, 2018; Villegas \& Lucas, 2002).

3. The preparation of teacher educators, particularly those involved in the teacher candidates clinical experience, is crucial to the preparation of teacher candidates to work with diverse learners through strengths-based perspectives (Thomas-Alexander \& Harper, 2017; Underwood \& Mensah, 2018; Vass, 2017).

I argue that science teachers could be better equipped to implement the NGSS in ways that are culturally sustaining for their traditionally underserved learners if they are adequately prepared with a foundation in culturally sustaining pedagogies when they are teacher candidates.

Teacher candidates often cite their clinical practice experience (White \& Forgasz, 2016) and the role of their cooperating teachers (Clarke et al., 2014) as one of the most influential aspects of their teacher preparation programs. I contend that capacity building among those teacher educators, such as cooperating teachers, responsible for STEM teacher preparation is essential in supporting the implementation of the NGSS in ways that are culturally sustaining for traditionally underserved learners. While the teacher educator participants in this study — science cooperating teachers in the River School District - stated that they did not feel prepared in these ways during their teacher 
preparation programs, the preparedness these professionals did express in this study around the components of the CSST conceptual framework offer great promise for the next generation of science teachers. The cooperating teachers who participated in this study serve as mentors for the next generation of science teachers within the context of the shifting landscape of STEM education. Under their guidance, the next generation of science teachers, who could also become the next generation of science cooperating teachers, could be prepared to implement the new standards in ways that are culturally sustaining for traditionally underserved learners. With more science teacher candidates prepared to use strengths-based approaches such as culturally sustaining pedagogiesones that honor students of color funds of knowledge-, more of the next generation of students of color can expand their science literacy skills (Meyer \& Crawford, 2011; Rodriguez, 2015). Expanding students' science literacies through their funds of knowledge can empower them to engage in the process of working toward solutions to dire problems that are likely to continue to affect them and their communities.

When schools provide students of color opportunities to expand their science literacy skills through their funds of knowledge, they increase opportunities for students of color to participate in developing solutions to significant problems that can affect them and their communities. This approach affords students of color with opportunities to access fast growing and high paying STEM careers. The challenges local and global communities face now and, in the future, are dire and many require STEM solutions. Such challenges impact communities of color at higher rates, making opportunities in schooling for students of color to expand their science literacy skills through their funds 
of knowledge critical. All members to the next generation deserve to be a part of developing solutions to the challenges that impact them and their communities. 
References

Aikenhead, G. S., \& Jegede, O. J. (1999). Cross-cultural science education: A cognitive explanation of a cultural phenomenon. Journal of Research in Science Teaching, $36,269-287$.

Anderson, L. M., \& Stillman, J. A. (2013). Student teaching's contribution to preservice teacher development: A review of research focused on the preparation of teachers for urban and high-needs contexts. Review of Educational Research, 83, 3-69.

Ball, D. L., Sleep, L., Boerst, T., \& Bass, H. (2009). Combining the development of practice and the practice of development in teacher education. Elementary School Journal, 109, 458-474.

Bandura, A. (2002). Social cognitive theory in cultural context. Applied Psychology: An International Review, 51, 269-290.

Braaten, M., \& Sheth, M. (2017). Tensions teaching science for equity: Lessons learned from the case of Ms. Dawson. Science Education, 101, 134-164.

Bransford, J., Brown, A. L., \& Cocking, R. R. (2000). How people learn: Brain, mind, experience, and school (Expanded ed.). Washington, DC: National Research Council.

Bettez, S., Aguilar-Valdez, J., Carlone, H., \& Cooper, J. (2011). On negotiating White science: A call for cultural relevance and critical reflexivity. Cultural Studies of Science Education, 6, 941-950.

Boone, H. N., \& Boone, D. A. (2012). Analyzing Likert data. Journal of Extension, 50(2), 1-5. (Article \#2TOT2) 
Britner, S. L., \& Pajares, F. (2006). Sources of science self-efficacy beliefs of middle school students. Journal of Research in Science Teaching, 43, 485-499.

Brown, J. C. (2017). A metasynthesis of the complementarity of culturally responsive and inquiry-based science education in K-12 settings: Implications for advancing equitable science teaching and learning. Journal of Research in Science Teaching, $54,1143-1173$.

Brown, J. C., \& Crippen, K. J. (2016a). Designing for culturally responsive science education through professional development. International Journal of Science Education, 38, 470-492.

Brown, J. C., \& Crippen, K. J. (2016b). The growing awareness inventory: Building capacity for culturally responsive science and mathematics with a structured observation protocol. School Science and Mathematics, 116, 127-138.

Bryan, L. A., \& Atwater, M. M. (2002). Teacher beliefs and cultural models: A challenge for science teacher preparation programs. Science Education, 86, 821-839. doi: 10.1002/sce. 10043

Bybee, R. (2014). NGSS and the next generation of science teachers. Journal for Science Teacher Education, 25, 211-221. doi: 10.1007/s10972-014-9381-4

Bybee, R., Taylor, J., Gardner, A., Van Scotter, P., Powell, J. C., Westbrook, A., \& Landes, N. (2006). The BSCS 5E instructional model: Origins and effectiveness. Colorado Springs, CO: BSCS.

Carifio, J., \& Perla, R. (2008). Resolving the 50-year debate around using and misusing Likert scales. Medical Education, 42, 1150-1152. 
Civil, M. (2016). STEM learning research through a funds of knowledge lens. Cultural Studies of Science Education, 11, 41-59.

Clarke, A., Triggs, V., \& Nielsen, W. (2014). Cooperating teacher participation in teacher education: A review of the literature. Review of Educational Research, 84, 163202. doi.org/10.3102/0034654313499618

Costa, V. B. (1995). When science is "another world": Relationships between worlds of family, friends, school, and science. Science Education, 79, 313-333.

Creswell, J. W., \& Plano-Clark, V. L. (2007). Designing and conducting mixed methods research. Thousand Oaks, CA: SAGE.

Creswell, J. W. (2014). Research design: Qualitative, quantitative, and mixed methods approaches $\left(4^{\text {th }}\right.$ ed. $)$. Los Angeles, CA: SAGE.

Cross, C. T. (2014). Political education: Setting the course for state and federal policy $\left(2^{\text {nd }}\right.$ ed.). New York, NY: Teachers College Press.

Darling-Hammond, L. (2006). Constructing $21^{\text {st }}$-century teacher education. Journal of Teacher Education, 57, 300-314.

Delgado Bernal, D., \& Villalpando, O. (2016). An apartheid of knowledge in academia. The struggle over the "legitimate" knowledge of faculty of color. In E. Taylor, D. Gillborn, \& G. Ladson-Billings (Eds.), Foundations of critical race theory $\left(2^{\text {nd }}\right.$ ed., pp. 77-92). New York, NY: Routledge.

Dewey, J. (1933). How we think. Buffalo, NY: Prometheus Books.

Dodo Seriki, V. (2018). Toward a framework for culturally relevant inquiry-based science pedagogy. In G. Prime (Ed.), Centering race in the STEM education of 
African-American K-12 learners. (pp. 151-170). Bern, Switzerland: Peter Lang US.

Dole, S., Bloom, L., \& Kowalske, K. (2015). Transforming pedagogy: Changing perspectives from teacher-centered to learner-centered. Interdisciplinary Journal of Problem-Based Learning, 10(1). doi.org/10.7771/1541-5015.1538

Dominguez, M. (2017). Decolonial teacher education as a needed bridge to culturally sustaining and revitalizing pedagogies. In D. Paris, \& S. H. Alim (Eds.), Culturally sustaining pedagogies: Teaching and learning for justice in a changing world (pp. 225-246). New York: NY. Teachers College Press.

Dover, A. G., \& Schultz, B. D. (2016). Troubling the edTPA: Illusions of objectivity and rigor. The Educational Forum, 80, 95-106.

doi.org/10.1080/00131725.2015.1102368

Farber, D. (2017). 2050: The challenges ahead. Retrieved from http://legal-planet.org/2017/03/02/2050-the-challenges-ahead/

Farber, D. A., \& Sherry, S. (2016). Telling stories out of school. In E. Taylor, D. Gillborn, \& G. Ladson-Billings (Eds.), Foundations of critical race theory $\left(2^{\text {nd }}\right.$ ed., pp. 77-92). New York, NY: Routledge.

Ferber, T., \& Nillas, L. (2010). Through the eyes of students-teachers: Successes and challenges in field teaching experiences. National Teacher Education Journal, $3(2), 61-86$.

Field, A. (2018). Discovering statistics using IBM SPSS statistics (5 ${ }^{\text {th }}$ ed.). London, England: SAGE. 
Foronda, C., Baptiste, D. L., Reinholdt, M. M., \& Ousman, K. (2016). Cultural humility: A concept analysis. Journal of Transcultural Nursing, 27, 210-217.

Fowler, F. J. (2009). Applied social research methods: Survey research methods $\left(4^{\text {th }}\right.$ ed.). Thousands Oaks, CA: SAGE.

Fowler, F. C. (2013). Policy studies for educational leaders: An introduction (4 ${ }^{\text {th }}$ ed.). Boston, MA: Pearson.

Gay, G. (2002). Culturally responsive teaching in special education for ethnically diverse students: Setting the stage. International Journal of Qualitative Studies, 15, 613629.

Gay, G. (2010). Culturally responsive teaching: Theory, practice, \& research ( $2^{\text {nd }}$ ed.). New York, NY: Teachers College Press.

Geier, R., Blumenfield, P. C., Marx, R., Krajcik, J. S., Fishman, B., Soloway, E., \& ClayChambers, J. (2008). Standardized test outcomes for students engaged in inquirybased science curricula in the context of urban reform. Journal of Research in Science Teaching, 45, 922-939.

George Fox University. (2016). Become a teacher. Retrieved from http://www.georgefox.edu/education/become-a-teacher/index.html

González, N., Moll, L. C., \& Amanti, C. (2005). Funds of knowledge: Theorizing practice in households, communities, and classrooms. Mahwah, NJ: Erlbaum. Goodwin, A. L., Smith, L., Souto-Manning, M., Cheruvu, R., Tan, M., Reed, R., \& Taveras, L. (2014). What should teacher educators know and be able to do? 
Perspectives from practicing teacher educators. Journal of Teacher Education, 65, $285-302$.

Grossman, P. (2010). Learning to practice: The design of clinical experience in teacher preparation (Policy brief). Retrieved from http://www.nea.org/assets/docs/Clinical_Experience_-_Pam_Grossman.pdf

Guest, G., MacQueen, K. M., \& Namey, E. E. (2012). Applied thematic analysis. Los Angeles, CA: SAGE.

Haag, S., \& Megowan, C. (2015). Next generation science standards: A national mixedmethods study on teacher readiness: National study on NGSS readiness. School Science and Mathematics, 115, 416-426.

Harris, C. J., Penuel, W. R., D’Angelo, C. M., DeBarger, A. H., Gallagher, L. P., Kennedy, C. A., \& Krajcik, J. S. (2015). Impact of project-based curriculum materials on student learning in science: Results of a randomized controlled trial. Journal of Research in Science Teaching, 52, 1362-1385.

Hawkins, D. B. (2016, February 1). Diverse student populations are in the classroom. Are we prepared to meet their needs? National Education Association Today, 1(1). Retrieved from http://www.nea.org/home/66241.htm

Hsiao, Y. J. (2015). The Culturally Responsive Teacher Preparedness Scale: An exploratory study. Contemporary Issues in Education Research, 8, 241-250.

Hook, J. N., Davis, D. E., Owen, J., Worthington, E. L., \& Utsey, S. O. (2013). Cultural humility: Measuring openness to culturally diverse clients. Journal of Counseling Psychology, 60, 353-366. 
Januszyk, R., Miller, E. C., \& Lee, O. (2016) Addressing student diversity and equity: The "Next Generation Science Standards" are leading a new wave of reform. Science and Children, 53(8), 28-31.

Johnson, C. C. (2011). The road to culturally relevant science: Exploring how teachers navigate change in pedagogy. Journal of Research in Science Teaching, 48, 170198.

Kang, E. J. S., Bianchini, J. A., \& Kelly, G. J. (2013). Crossing the border from science student to science teacher: Preservice teachers' views and experiences learning to teach inquiry. Journal of Science Teacher Education, 24, 427-447.

Kanter, D. E., \& Konstantopoulos, S. (2010). The impact of project-based science curriculum on minority student achievement, attitudes, and careers: The effects of teacher content and pedagogical content knowledge and inquiry-based practices. Science Education, 94, 855-887.

Kissau, S., Hart, L.C., \& Algozzine, B. (2017). Investigating the impact of edTPA professional development on classroom practice and student teaching experience. Journal of Teacher Education, 70, 102-114.

Kloser, M. (2014). Identifying a core set of science teaching practices: A delphi expert panel approach. Journal of Research in Science Teaching, 51, 1185-1217.

Krajcik, J. (2015). Three dimensional instruction: Using a new type of teaching in the science classroom. Science and Children, 53(3), 6-8. 
Krosnick, J. A., \& Presser, S. (2010). Question and questionnaire design. In P. V. Marsden \& J. D. Wright (Eds.), Handbook of survey research (2 ${ }^{\text {nd }}$ ed., pp. 263313). Bingley, UK: Emerald Group.

Ladson-Billings, G. (1995). But that's just good teaching! The case for culturally relevant pedagogy. Theory Into Practice, 34, 159-165.

Ladson-Billings, G. (2009). Just what is critical race theory and what's it doing in a nice field like education? In E. Taylor, D. Gillborn, \& G. Ladson-Billings (Eds.), Foundations of critical race theory in education (pp. 17-36). New York, NY: Routledge.

Landivar, L. C. (2013). Disparities in STEM employment by sex, race, and Hispanic origin. [American Community Survey Reports, ACS-24]. Washington, DC: U.S. Census Bureau.

Laughter, J. C., \& Adams, A. D. (2012). Culturally relevant science teaching in middle school. Urban Education, 47, 1106-1134.

Litowitz, D. E. (1999). Some critical thoughts on critical race theory. Notre Dame Law Review, 72, 503-529.

Lortie, D. C. (1975). Schoolteacher: A sociological study. Chicago, IL: University of Chicago Press.

Luft, J. A., da Cunha, T., \& Allison, A. (1998). Increasing the participation of minority students in science: A study of two teachers. Retrieved from ERIC database. (ED418944) 
Lund, D. E., \& Lee, L. (2015). Fostering cultural humility among pre-service teachers: Connecting with children and youth of immigrant families through servicelearning. Canadian Journal of Education, 38(2), 1-30.

Marion, R., \& Gonzales, L. D. (2014). Leadership in education: Organizational theory for the practitioner ( $2^{\text {nd }}$ ed.). Long Grove, IL: Waveland Press.

Marshall, J. C., Horton, B., \& Smart, J. (2009). 4E X 2 Instructional model: Uniting three learning constructs to improve praxis in science and mathematics classrooms. Journal of Science Teacher Education, 20, 501-516.

Marshall, J. C., \& Smart, J. B. (2013). Teachers' transformation to inquiry-based instructional practice. Creative Education, 4(2), 132-142.

Marshall, J. C., Smart, J., \& Horton, R. M. (2011). Tracking perceived and observed growth of inquiry practice: A formative plan to improve professional development experiences. Science Educator, 20(1), 12-22.

Marx, R., \& Harris, C. (2006). No child left behind and science education: Opportunities, challenges, and risks. The Elementary School Journal, 106, 467-478.

Matsko, K. K., Ronfeldt, M., Nolan, H. G., Klugman, J., Reininger, M., \& Brockman, S. L. (2018). Cooperating teacher as model and coach: What leads to student teachers' perceptions of preparedness? Journal of Teacher Education, 1-12.

Mayer, S. J. (2008). Dewey's dynamic integration of Vygotsky and Piaget. Education and Culture, 24(2), 6-24. 
Meyer, X., \& Crawford, B. A. (2011). Teaching science as a cultural way of knowing: Merging authentic inquiry, nature of science, and multicultural strategies. Cultural Studies of Science Education, 6, 525-547.

Merriam, S. B., \& Tisdell, E. J. (2016). Qualitative research: A guide to design and implementation. San Francisco, CA: Jossey-Bass.

Miles, M. B., Huberman, M. A., \& Saldaña, J. (2014). Qualitative data analysis: a methods sourcebook ( $3^{\text {rd }}$ ed. $)$. Thousand Oaks, CA: SAGE.

Moje, E. B., Ciechanowski, K. M., Kramer, K., Ellis, L., Carrillo, R., \& Collazo, T. (2004). Working toward third space in content area literacy: An examination of everyday funds of knowledge and Discourse. Reading Research Quarterly, 39, $38-70$.

Morgan, D. L. (1996). Focus groups. Annual Review of Sociology, 22, 129-152.

Morgan, D. L. (2007). Paradigms lost and paradigms regained: Methodological implications of combining qualitative and quantitative methods. Journal of Mixed Methods Research. 1, 48-76.

Morgan, D. L. (2014). Integrating qualitative and quantitative methods: A pragmatic approach. Thousand Oaks, CA: SAGE.

Moseley, C., Bilica, K., Wandless, A., \& Gdvoin, R., (2014). Exploring the relationship between teaching efficacy and cultural efficacy of novice science teachers in high-needs schools. School Science and Mathematics, 114, 315-325.

National Center for Education Statistics. (2017). Racial/ethnic enrollment in public schools. Retrieved from https://nces.ed.gov/programs/coe/indicator_cge.asp 
National Research Council. (2011). Successful K-12 STEM education: Identifying approaches in science, technology, engineering, and math. Washington, DC: The National Academy Press.

National Research Council. (2012). A framework for K-12 science education: Practices, crosscutting concepts, and core ideas. Washington, DC: The National Academy Press.

Paris, D. (2012). Culturally sustaining pedagogy: A needed change in stance, terminology, and practice. Educational Researcher, 41, 93-97.

Paris, D. (2016). On educating culturally sustaining teachers. Retrieved from http://www.teachingworks.org/images/files/TeachingWorks_Paris.pdf

Paris, D., \& Alim, S. H., (2017). Culturally sustaining pedagogies: Teaching and learning for justice in a changing world. New York: NY. Teachers College Press.

Powell, R., Cantrell, S., Gallardo Carter, Y., Cox, A., Powers, S., Rightmyer, E. C., \& Wheeler, T. (2012). Culturally Responsive Instruction Observation Protocol (revised). Lexington, KY: Collaborative Center for Literacy Development.

Powell, R., Cantrell, S. C., Malo-Juvera, V., \& Correll, P. (2016). Operationalizing culturally responsive instruction: Preliminary findings of CRIOP research. Teachers College Record, 118, 1-46.

Puzio, K., Newcomer, S., Pratt, K., McNeely, K., Jacobs, M., \& Hooker, S. (2017). Creative failures in culturally sustaining pedagogy. Language Arts, 94(4), 223233. 
Rodriguez, A. J. (1998). Strategies for counterresistance: Toward sociotransformative constructivism and learning to teach science for diversity and for understanding. Journal of Research in Science Teaching, 35, 589-622.

Rodriguez, A. J. (2015). What about a dimension of engagement, equity, and diversity practices? A critique of the next generation science standards. Journal of Research in Science Teaching, 52, 1031-1051.

Ronfeldt, M., Brockman, S. L., \& Campbell, S. L. (2018). Does cooperating teachers' instructional effectiveness improve preservice teachers' future performance? Educational Researcher, 47, 405-418.

Saldaña, J. (2013). The coding manual for qualitative researchers $\left(2^{\text {nd }}\right.$ ed.). Thousand Oaks, CA: SAGE.

Singh, K., Granville, M., \& Dika, S. (2002). Mathematics and science achievement: Effects on motivation, interest, and academic engagement. The Journal of Educational Research, 95, 323-332.

Siwatu, K. O., Chesnut, S. R., Alejandro, A. Y., \& Young, H. A. (2016). Examining preservice teachers' culturally responsive teaching self-efficacy doubts. The Teacher Educator, 51, 277-296.

Sleeter, C. E. (2017). Critical race theory and the whiteness of teacher education. Urban Education, 52, 155-169.

Smith, L. T. (2005). On tricky ground: Researching the native in the age of uncertainty. In N. K. Denzin \& Y. S. Lincoln (Eds.), The SAGE handbook of qualitative research ( $3^{\text {rd }}$ ed., pp. 85-107). Thousand Oaks, CA: SAGE. 
Smith, R., \& Dearborn, G. (2016). Conscious classroom management: Unlocking the secrets of great teaching ( $2^{\text {nd }}$ ed.). San Rafael, CA: Conscious Teaching.

Smith-Maddox, R., \& Solorzano, D. G. (2002). Using critical race theory, Paulo Freire's problem-posing method, and case study research to confront race and racism in education. Qualitative Inquiry, 8, 66-84.

Swennen, J. M. H., Jones, K., \& Volman, M. L. L. (2010). Teacher educators: Their identities, sub-identities, and implications for professional development. Professional Development in Education, 36, 131-148.

Tan, E., \& Barton, A. C. (2010). Transforming science learning and student participation in sixth grade science: A case study of a low-income, urban, racial minority classroom. Equity \& Excellence in Education, 43, 38-55.

Taylor, E., Gillborn, D., \& Ladson-Billings, G. (2009). Foundations of critical race theory in education. New York, NY: Routledge.

Thomas-Alexander, S., \& Harper, B. E. (2017). Cleaning up the clinic: Examining mentor teachers' perceptions of urban classrooms and culturally responsive teaching. Multicultural Learning and Teaching, 12(1), 49-65.

Tinkler, A. S., \& Tinkler, B. (2016). Enhancing cultural humility through critical servicelearning in teacher preparation. Multicultural Perspectives, 18, 192-201.

Underwood, J. B., \& Mensah, F. M. (2018). An investigation of science teacher educators' perceptions of culturally relevant pedagogy. Journal of Science Teacher Education, 29, 46-64. 
Vass, G. (2017). Preparing for culturally responsive schooling: Initial teacher educators into the fray. Journal of Teacher Education, 68, 451-462.

Villegas, A. M., \& Lucas, T. (2002). Preparing culturally responsive teachers: Rethinking the curriculum. Journal of Teacher Education, 53, 20-32.

Vygotsky, L. S. (1978). Mind in society: The development of higher psychological processes. Cambridge, MA: Harvard University Press.

White, S., \& Forgasz, R. (2016) The practicum: The place of experience?. In J. Loughran, \& M. Hamilton M. (Eds.), (eds, International Handbook of Teacher Education (pp. 231-266). Singapore: Springer.

Windschitl, M., Thompson, J., Braaten, M., \& Stroupe, D. (2012). Proposing a core set of instructional practices and tools for teachers of science. Science Education, 96, 878-903.

Windschitl, M. A., \& Stroupe D. (2017). The three-story challenge: Implications of the Next Generation Science standards for teacher preparation. Journal of Teacher Education, 68, 251-261.

100kin10. (n.d). Our story. Retrieved from https://100kin10.org/about 


\section{Appendix A}

The Culturally Responsive Teacher Preparedness Scale (Hsiao, 2015)

Directions:

The following is a list of competencies of culturally responsive teaching. There are three areas: curriculum and instruction, relationship and expectation establishment, and group belonging formation. Please rate each competency by marking the appropriate box to indicate your preparedness of these competencies. The options range between "Unprepared" (1) to "Fully prepared" (6).

\begin{tabular}{|c|c|c|}
\hline \multicolumn{3}{|c|}{ 1. Curriculum and Instruction } \\
\hline I am able to: & $\begin{array}{l}1 \\
\text { Unprepared }\end{array}$ & $\begin{array}{l}6 \\
\text { Fully } \\
\text { Prepared }\end{array}$ \\
\hline $\begin{array}{l}\text { 1. find ways to support language acquisition and } \\
\text { enhance culturally and linguistically diverse } \\
\text { students' comprehension of classroom tasks. }\end{array}$ & $\square$ & $\square$ \\
\hline $\begin{array}{l}\text { 2. review and assess curricula and instructional } \\
\text { materials to determine their multicultural } \\
\text { strengths and weaknesses, and relevance to } \\
\text { students' interests and instructional needs, and } \\
\text { revise them if necessary. }\end{array}$ & $\square$ & $\square$ \\
\hline $\begin{array}{l}\text { 3. develop a repertoire of instructional examples } \\
\text { that are culturally familiar to students to serve as } \\
\text { a scaffold for learning. }\end{array}$ & $\square$ & $\square$ \\
\hline $\begin{array}{l}\text { 4. infuse the curriculum and thematic units with } \\
\text { the culture of students represented in the } \\
\text { classroom. }\end{array}$ & $\square$ & $\square$ \\
\hline $\begin{array}{l}\text { 5. Utilize a variety of instructional methods to } \\
\text { match students' learning preferences in learning } \\
\text { the subject matter, and maintaining their } \\
\text { attention and interest in learning. }\end{array}$ & $\square$ & $\square$ \\
\hline $\begin{array}{l}\text { 6. assess culturally diverse students' readiness, } \\
\text { intellectual and academic strengths and } \\
\text { weaknesses, and development needs. }\end{array}$ & $\square$ & $\square$ \\
\hline
\end{tabular}




\begin{tabular}{|l|l|l|l|l|l|}
\hline $\begin{array}{l}\text { 7. use a variety of assessment techniques, such as } \\
\text { self-assessment, portfolios, and so on, to evaluate } \\
\text { students' performance in favor of cultural } \\
\text { diversity. }\end{array}$ & $\square$ & & & & $\square$ \\
\hline $\begin{array}{l}\text { 8. design assessments to complement the } \\
\text { culturally responsive pedagogical strategies that } \\
\text { were employed during instruction. }\end{array}$ & $\square$ & & & $\square$ \\
\hline
\end{tabular}

\begin{tabular}{|c|c|c|}
\hline \multicolumn{3}{|c|}{ 2. Relationship and Expectation Establishment } \\
\hline I am able to: & $\begin{array}{l}1 \\
\text { Unprepared }\end{array}$ & $\begin{array}{l}6 \\
\text { Fully } \\
\text { Prepared }\end{array}$ \\
\hline $\begin{array}{l}\text { 1. know how to communicate with culturally } \\
\text { diverse students and their parents or guardians. }\end{array}$ & $\square$ & $\square$ \\
\hline $\begin{array}{l}\text { 2. structure classroom-based meetings that are } \\
\text { comfortable for parents. }\end{array}$ & $\square$ & $\square$ \\
\hline $\begin{array}{l}\text { 3. foster meaningful and supportive relationships } \\
\text { with parents and families, and actively involve } \\
\text { them in their students' learning. }\end{array}$ & $\square$ & $\square$ \\
\hline $\begin{array}{l}\text { 4. use non-traditional discourse styles with } \\
\text { culturally diverse students in an attempt to } \\
\text { communicate in culturally responsive ways. }\end{array}$ & $\square$ & $\square$ \\
\hline $\begin{array}{l}\text { 5. establish expectations for appropriate } \\
\text { classroom behavior in considering students' } \\
\text { cultural backgrounds to maintain a conducive } \\
\text { learning environment. }\end{array}$ & $\square$ & $\square$ \\
\hline $\begin{array}{l}\text { 6. communicate expectations of success to } \\
\text { culturally diverse students. }\end{array}$ & $\square$ & $\square$ \\
\hline
\end{tabular}

\begin{tabular}{|l|l|l|l|l|l|}
\hline \multicolumn{7}{|c|}{ 3. Group Belonging Formation } \\
\hline I am able to: & $\begin{array}{l}1 \\
\text { Unprepared }\end{array}$ & & & $\begin{array}{l}6 \\
\text { Fully } \\
\text { Prepared }\end{array}$ \\
\hline
\end{tabular}




\begin{tabular}{|l|l|l|l|l|l|}
\hline $\begin{array}{l}\text { 1. create a warm, supporting, safe, and secure } \\
\text { classroom environment for culturally diverse } \\
\text { students. }\end{array}$ & $\square$ & & & & $\square$ \\
\hline $\begin{array}{l}\text { 2. create a community of learners by } \\
\text { encouraging students to focus on collective } \\
\text { work, responsibility, and cooperation. }\end{array}$ & $\square$ & & & & $\square$ \\
\hline $\begin{array}{l}\text { 3. develop and maintain positive, meaningful, } \\
\text { caring, and trusting relationships with students. }\end{array}$ & $\square$ & & & $\square$ \\
\hline $\begin{array}{l}\text { 4. provide students with knowledge and skills } \\
\text { needed to function in mainstream culture. }\end{array}$ & $\square$ & & & & $\square$ \\
\hline
\end{tabular}

Hsiao, Y.-J. (2015). The Culturally Responsive Teacher Preparedness Scale: An exploratory study. Contemporary Issues in Education Research, 8, 241-250. 
Appendix B

\section{Culturally Sustaining Science Teaching Preparedness Survey (adapted from Hsiao, 2015)}

Directions for survey:

The following survey contains two parts related to your preparedness as a cooperating teacher working to support science teacher candidates. The items and questions included in the survey are not an exhaustive or prescriptive set of ways to think about serving traditionally underserved learners in science classrooms but rather one frame used in this research.

- Part 1 starts with an open-ended prompt asking you to describe the ways you feel prepared to support science teacher candidates to use pedagogies that are culturally sustaining for traditionally underserved learners. Then there is a list of items, grouped into three focus areas: curriculum, instruction, and relationships. For each item, please select your current level of preparedness. The options range between "Unprepared" (1) and "Fully prepared" (6). For each focus area, also please describe your thoughts about the ratings you selected.

- Part 2 includes a series of demographic questions meant to characterize your experience as a teacher educator. For each question, please select the range that applies to you including this year.

After an initial analysis of the results, I would like to host a focus group interview for you to have a chance to review and comment on the findings. If you would be willing to participate in that focus group interview, please indicate that at the end of the survey. Additionally, please indicate if you would like a summary of the results of the research study sent to you. If you wish to participate in this survey, please click on the link. By clicking on the next button below, I am indicating that I am 18 years of age or older and have read this consent form and am willing to participate in the research activity described above. Thank you for your willingness to contribute to the survey data for this research study.

\section{Part 1. Culturally sustaining science teaching item ratings and open-ended responses}

In what ways do you feel prepared to support science teacher candidates to use pedagogies that are culturally sustaining for traditionally underserved learners?

\begin{tabular}{|c|c|c|c|c|c|c|}
\hline \multicolumn{7}{|c|}{ Curriculum } \\
\hline $\begin{array}{l}\text { I am currently } \\
\text { to } \\
\text { support } \\
\text { science } \\
\text { teacher } \\
\text { candidates to: }\end{array}$ & $\begin{array}{l}1 \\
\text { Unprepared }\end{array}$ & $\begin{array}{l}2 \\
\text { Somewhat } \\
\text { Unprepared }\end{array}$ & $\begin{array}{l}3 \\
\text { Somewhat } \\
\text { Prepared }\end{array}$ & $\begin{array}{l}4 \\
\text { Prepared }\end{array}$ & $\begin{array}{l}5 \\
\text { Highly } \\
\text { Prepared }\end{array}$ & $\begin{array}{l}6 \\
\text { Fully } \\
\text { Prepared }\end{array}$ \\
\hline
\end{tabular}




\begin{tabular}{|c|c|c|c|c|c|c|}
\hline $\begin{array}{l}\text { 1.1 evaluate } \\
\text { science } \\
\text { curricula and } \\
\text { instructional } \\
\text { materials to } \\
\text { determine } \\
\text { their } \\
\text { multicultural } \\
\text { strengths and } \\
\text { weaknesses, } \\
\text { relevance to } \\
\text { students' } \\
\text { interests and } \\
\text { instructional } \\
\text { needs, and } \\
\text { revise them if } \\
\text { necessary. }\end{array}$ & $\square$ & $\square$ & $\square$ & $\square$ & $\square$ & $\square$ \\
\hline $\begin{array}{l}\text { 1.2. develop a } \\
\text { repertoire of } \\
\text { examples in } \\
\text { the science } \\
\text { curriculum } \\
\text { that are } \\
\text { culturally } \\
\text { familiar to } \\
\text { students to } \\
\text { scaffold } \\
\text { learning. }\end{array}$ & $\square$ & $\square$ & $\square$ & $\square$ & $\square$ & $\square$ \\
\hline $\begin{array}{l}\text { 1.3. infuse } \\
\text { the science } \\
\text { curriculum, } \\
\text { including } \\
\text { units and } \\
\text { lessons, with } \\
\text { the culture of } \\
\text { students } \\
\text { represented in } \\
\text { the } \\
\text { classroom. }\end{array}$ & $\square$ & $\square$ & $\square$ & $\square$ & $\square$ & $\square$ \\
\hline $\begin{array}{l}\text { 1.4. include a } \\
\text { variety of } \\
\text { instructional } \\
\text { methods to } \\
\text { match }\end{array}$ & $\square$ & $\square$ & $\square$ & $\square$ & $\square$ & $\square$ \\
\hline
\end{tabular}




\begin{tabular}{|c|c|c|c|c|c|c|}
\hline $\begin{array}{l}\text { students' } \\
\text { learning } \\
\text { preferences, } \\
\text { and maintain } \\
\text { their attention } \\
\text { and interest in } \\
\text { science. }\end{array}$ & & & & & & \\
\hline $\begin{array}{l}\text { 1.5. design } \\
\text { science } \\
\text { curriculum } \\
\text { that includes } \\
\text { students } \\
\text { obtaining, } \\
\text { evaluating, } \\
\text { and } \\
\text { communicatin } \\
\text { g information } \\
\text { about systems } \\
\text { of power, } \\
\text { privilege, and } \\
\text { marginalizati } \\
\text { on. }\end{array}$ & $\square$ & $\square$ & $\square$ & $\square$ & $\square$ & $\square$ \\
\hline
\end{tabular}

Please describe your thoughts about the ratings you selected within the curriculum section.

\begin{tabular}{|c|c|c|c|c|c|c|}
\hline \multicolumn{7}{|c|}{ Instruction } \\
\hline $\begin{array}{l}\text { I am currently } \\
\text { to } \\
\text { support } \\
\text { science } \\
\text { teacher } \\
\text { candidates to: }\end{array}$ & $\begin{array}{l}1 \\
\text { Unprepared }\end{array}$ & $\begin{array}{l}2 \\
\text { Somewhat } \\
\text { Unprepared }\end{array}$ & $\begin{array}{l}3 \\
\text { Somewhat } \\
\text { Prepared }\end{array}$ & $\begin{array}{l}4 \\
\text { Prepared }\end{array}$ & $\begin{array}{l}5 \\
\text { Highly } \\
\text { Prepared }\end{array}$ & $\begin{array}{l}6 \\
\text { Fully } \\
\text { Prepared }\end{array}$ \\
\hline $\begin{array}{l}\text { 2.1. find ways } \\
\text { to enhance } \\
\text { culturally and } \\
\text { linguistically } \\
\text { diverse } \\
\text { students' } \\
\text { comprehensio } \\
\mathrm{n} \text { and use of } \\
\text { science } \\
\text { related } \\
\text { content, } \\
\text { concepts, }\end{array}$ & $\square$ & $\square$ & $\square$ & $\square$ & $\square$ & $\square$ \\
\hline
\end{tabular}




\begin{tabular}{|c|c|c|c|c|c|c|}
\hline $\begin{array}{l}\text { vocabulary, } \\
\text { and skills. }\end{array}$ & & & & & & \\
\hline $\begin{array}{l}\text { 2.2. use a } \\
\text { variety of } \\
\text { linguistic } \\
\text { styles with } \\
\text { culturally } \\
\text { diverse } \\
\text { students in an } \\
\text { attempt to } \\
\text { communicate } \\
\text { in culturally } \\
\text { responsive or } \\
\text { sustaining } \\
\text { ways during } \\
\text { science } \\
\text { instruction. }\end{array}$ & $\square$ & $\square$ & $\square$ & $\square$ & $\square$ & $\square$ \\
\hline $\begin{array}{l}\text { 2.3. create a } \\
\text { community of } \\
\text { learners by } \\
\text { encouraging } \\
\text { students to } \\
\text { focus on } \\
\text { collective } \\
\text { work, } \\
\text { responsibility, } \\
\text { and } \\
\text { cooperation } \\
\text { when learning } \\
\text { science. }\end{array}$ & $\square$ & $\square$ & $\square$ & $\square$ & $\square$ & $\square$ \\
\hline $\begin{array}{l}\text { 2.4. provide } \\
\text { students with } \\
\text { knowledge } \\
\text { and skills } \\
\text { needed to } \\
\text { function in } \\
\text { mainstream } \\
\text { culture of } \\
\text { science and to } \\
\text { consider the } \\
\text { ways various } \\
\text { cultural } \\
\text { groups, } \\
\text { including }\end{array}$ & $\square$ & $\square$ & $\square$ & $\square$ & $\square$ & $\square$ \\
\hline
\end{tabular}




\begin{tabular}{|c|c|c|c|c|c|c|}
\hline $\begin{array}{l}\text { their own } \\
\text { contribute to } \\
\text { science. }\end{array}$ & & & & & & \\
\hline $\begin{array}{l}2.5 \text {. assist } \\
\text { students in } \\
\text { developing } \\
\text { and using } \\
\text { models that } \\
\text { represent } \\
\text { various ways } \\
\text { of knowing } \\
\text { science based } \\
\text { on their } \\
\text { cultural } \\
\text { practices and } \\
\text { knowledge. }\end{array}$ & $\square$ & $\square$ & $\square$ & $\square$ & 口 & $\square$ \\
\hline
\end{tabular}

Please describe your thoughts about the ratings you selected within the instruction section.

\begin{tabular}{|c|c|c|c|c|c|c|}
\hline \multicolumn{7}{|c|}{ Relationships } \\
\hline $\begin{array}{l}\text { I am currently } \\
\text { to } \\
\text { support } \\
\text { science } \\
\text { teacher } \\
\text { candidates to: }\end{array}$ & $\begin{array}{l}1 \\
\text { Unprepared }\end{array}$ & $\begin{array}{l}2 \\
\text { Somewhat } \\
\text { Unprepared }\end{array}$ & $\begin{array}{l}3 \\
\text { Somewhat } \\
\text { Prepared }\end{array}$ & $\begin{array}{l}4 \\
\text { Prepared }\end{array}$ & $\begin{array}{l}5 \\
\text { Highly } \\
\text { Prepared }\end{array}$ & $\begin{array}{l}6 \\
\text { Fully } \\
\text { Prepared }\end{array}$ \\
\hline $\begin{array}{l}\text { 3.1. create a } \\
\text { warm, } \\
\text { supporting, } \\
\text { safe, and } \\
\text { secure } \\
\text { classroom } \\
\text { environment } \\
\text { for culturally } \\
\text { diverse } \\
\text { students to } \\
\text { learn science. }\end{array}$ & $\square$ & $\square$ & $\square$ & $\square$ & $\square$ & $\square$ \\
\hline $\begin{array}{l}\text { 3.2. develop } \\
\text { and maintain } \\
\text { positive, } \\
\text { meaningful, } \\
\text { caring, and } \\
\text { trusting }\end{array}$ & $\square$ & $\square$ & $\square$ & $\square$ & $\square$ & $\square$ \\
\hline
\end{tabular}




\begin{tabular}{|c|c|c|c|c|c|c|}
\hline $\begin{array}{l}\text { relationships } \\
\text { with students. }\end{array}$ & & & & & & \\
\hline $\begin{array}{l}\text { 3.3. establish } \\
\text { expectations } \\
\text { for } \\
\text { appropriate } \\
\text { classroom } \\
\text { behavior in } \\
\text { considering } \\
\text { students' } \\
\text { cultural } \\
\text { backgrounds } \\
\text { to maintain a } \\
\text { conducive } \\
\text { learning } \\
\text { environment. }\end{array}$ & $\square$ & $\square$ & $\square$ & $\square$ & $\square$ & $\square$ \\
\hline $\begin{array}{l}3.4 \text {. } \\
\text { communicate } \\
\text { expectations } \\
\text { of success to } \\
\text { culturally } \\
\text { diverse } \\
\text { students that } \\
\text { are grounded } \\
\text { in positive } \\
\text { perceptions of } \\
\text { all learners. }\end{array}$ & $\square$ & $\square$ & $\square$ & $\square$ & $\square$ & $\square$ \\
\hline $\begin{array}{l}\text { 3.5. guide } \\
\text { students to } \\
\text { construct } \\
\text { explanations } \\
\text { about } \\
\text { problems or } \\
\text { challenges } \\
\text { that impact } \\
\text { them and } \\
\text { their } \\
\text { communities. }\end{array}$ & $\square$ & $\square$ & $\square$ & $\square$ & $\square$ & $\square$ \\
\hline
\end{tabular}

Please describe your thoughts about the ratings you selected within the relationships section. 
Note: Adapted from Hsiao, Y.-J. (2015). The Culturally Responsive Teacher Preparedness Scale: An exploratory study. Contemporary Issues in Education Research, $8,241-250$.

\section{Part 2. Your experience as a teacher educator (cooperating teacher)}

1. How many years have you been a $6^{\text {th }}-12^{\text {th }}$-grade science teacher?

- $1-3$

- 4-6

- $7-9$

- $10-12$

- $13-15$

- $15+$

2. As a science teacher:

a. What subject(s) have you taught? (Biology, Chemistry, Physics, General Science, Environmental Science, Integrated Science)

b. What subject are you currently teaching?

c. How many years have you been teaching the subject you are currently teaching?

d. How many years have you been teaching at the school you currently teach?

3. How would you describe the school where you currently teach?

- Rural

- Suburban

- Urban

4. As you think about your most diverse class, how would you describe the student population in that class in terms of racial and linguistic diversity? (e.g. groups, percentages of each group)

Students of color:

English Language Learners:

5. As you think about all of your classes, how would you describe your current student population in terms of racial and linguistic diversity?

Students of color:

English Language Learners:

6. How many years have you served as a cooperating teacher with science teacher candidates at this school?

- 1-3

- 4-6

- 7-9

- $10-12$

- $13-15$

- $15+$ 
7. As a cooperating teacher working with science teacher candidates:

a. What subject(s) have you worked with science teacher candidates to teach?

b. What subject are you currently working with science teacher candidates to teach?

c. How many years have you been working with science teacher candidates on this subject?

d. How many years have you been working with science teacher candidates at the school where you currently teach?

8. What was your teacher preparation program? Check all that apply.

- Undergraduate

- Graduate

- Alternative Certification

9. How old are you?

- 20-25

- $25-30$

- $30-35$

- $35-40$

- $45-50$

- $50+$

10. How would you describe yourself in terms of racial and linguistic diversity?

Check all that apply.

- Culturally Diverse

- Linguistically Diverse

11. Should you feel comfortable, please share more about how you identify in terms of culture (including factors around gender, race, ethnicity, nationality, and language).

\section{Follow up preferences}

I would be willing to participate in a focus group interview: YES / NO

I would like the results of the study sent to me: YES / NO

\section{References}

Brown, J. C. (2017). A metasynthesis of the complementarity of culturally responsive and inquiry-based science education in K-12 settings: Implications for advancing equitable science teaching and learning. Journal of Research in Science Teaching, 54, 1143-1173.

Gay, G. (2010). Culturally responsive teaching: Theory, practice, \& research. New York, NY: Teachers College Press. 
Hsiao, Y.-J. (2015). The Culturally Responsive Teacher Preparedness Scale: An exploratory study. Contemporary Issues in Education Research, 8, 241-250.

Paris, D., \& Alim, S.H., (2017). Culturally sustaining pedagogies: Teaching and learning for justice in a changing world. New York: NY. Teachers College Press. 


\section{Appendix C \\ Email Script from Hsiao}

Hi Keelan,

Thank you for your email. I am glad to know what you are going to do for your dissertation research. I am willing to share my instrument with you. You are welcome to modify it to fit your study. Attached, please find the Word and Pdf document of the scale.

Feel free to let me know if you need anything else.

Good luck for your dissertation!!!

Best,

Yun-Ju

Yun-Ju Hsiao, Ph.D.

Assistant Professor of Special Education

Washington State University Tri-Cities

College of Education

2710 Crimson Way

Office TFLO 207L

Richland, WA 99354

509-372-7505

yhsiao@tricity.wsu.edu 
Appendix D

Email Script to Request Teacher Participation

Dear River School District Science Teacher,

I am writing to see if you would be willing to complete a short survey (15-20 minutes) for my dissertation research. Specifically, if you are currently serving as, or have in the past five school years served as, a cooperating teacher for a science teacher candidate I am seeking your participation.

The information for participation is presented in this email in two sections: Informed Consent and Directions for Survey. These elements are required by the Institutional Review Board for the protection of human subjects in research. You will be directed to the survey on the Qualtrics platform at the end of the second section.

Thank you for your time in helping us to understand the strengths that cooperating teachers like you bring to the preparation of science teacher candidates. Your input has tremendous value!

Sincerely,

Keelan LoFaro

\section{$\underline{\text { Informed Consent }}$}

You are invited to participate in this research study that aims to acknowledge and honor the vital role you play as a teacher educator who supports science teacher candidates. The information you provide will help to shed light on the ways cooperating teachers feel prepared to support science teacher candidates to use pedagogies that serve our traditionally underserved learners.

There are no foreseeable risks to participating in this research. Your participation in this survey is voluntary, you can withdraw your participation at any time, and there is no penalty for refusing to participate. Completing this survey should take approximately 1520 minutes of your time.

Information received through this survey will be kept confidential and secured on a password-protected computer. After three years, all information collected from this survey will be destroyed.

At the end of the survey, you will have an opportunity to indicate if you are willing to participate in a follow-up focus group interview to help strengthen the interpretation of the survey data analysis. If you choose to participate in the focus group, there is a risk to your confidentiality in that other focus group participants will hear your responses to the 
focus group interview questions. The focus group interview questions will not ask you about your individual preparedness like the survey does but rather will ask for your thoughts on an analysis of the survey data with respect to the strengths and areas of need indicated by cooperating teachers in your district. During the focus group interview, you will have the opportunity to choose to answer or not answer any of the questions posed.

If you have any questions about the survey or research, please feel free to contact a member of the research team: the principal investigator Micki Caskey, at caskeym@pdx.edu or the co-principal investigator Keelan LoFaro, atklofaro@pdx.edu.

\section{Directions for Survey}

The following survey contains two parts related to your preparedness as a cooperating teacher working to support science teacher candidates. The items and questions included in the survey are not an exhaustive or prescriptive set of ways to think about serving traditionally underserved learners in science classrooms but rather one frame used in this research.

Part 1 starts with an open-ended prompt. Then there is a list of items, grouped into three focus areas: curriculum, instruction, and relationships. For each item, please select your current level of preparedness between "Unprepared" (1) and "Fully prepared" (6) and describe your thoughts about the ratings you selected.

- $\quad$ Part 2 includes a series of demographic questions meant to characterize your experience as a teacher educator.

After an initial analysis of the results, I would like to host a focus group interview for you to have a chance to review and comment on the findings. If you would be willing to participate in that focus group interview, please indicate that at the end of the survey. Additionally, please indicate if you would like a summary of the results of the research study sent to you.

If you wish to participate in this survey, please click on the link below to complete the survey by March 18th. By clicking on the survey link, I am indicating that I am 18 years of age or older, have read the informed consent form and am willing to participate in the research activity described. Thank you for your willingness to contribute to the survey data for this research study.

Link to survey

Take the survey

Or copy and paste the URL below into your internet browser https://portlandstate.qualtrics.com/jfe/form/SV_8cRMcvsRayUj9oF 


\section{Appendix E}

Semi-Structure Focus Group Questions

(based on preliminary analysis of survey data)

\section{Overall Findings}

Quantitative Findings (Mean Ranks)

The survey results indicate that the components ranked in the following order from greatest to least with respect to perceived preparedness were (a) relationships $(M=4.68$, $S D=1.12)$, (b) curriculum $(M=4.15, S D=1.03)$, and (c) instruction $(M=4.10, S D=$ 1.01 ). Based on your experience as a cooperating teacher working to support teacher candidates to serve traditionally underserved learners, what you say about the accuracy of these overall quantitative results?

\section{Qualitative Findings}

The open coding of the survey's qualitative items revealed that science cooperating teachers did not feel they were originally prepared to use culturally relevant or responsive science teaching practices. Yet, they did feel a responsibility to support teacher candidates with these practices based on what they learned through their teaching experience or attending professional development training where they gathered resources and learned from others. Based on your experience as a cooperating teacher working to support teacher candidates to serve traditionally underserved learners in your district, what you say about the accuracy of these overall qualitative results? Is there anything you would expand upon or disagree with?

\section{Curriculum}

Quantitative Findings (Strengths)

The survey results indicated that the following construct of (1.4. include a variety of instructional methods to match students' learning preferences, and maintain their attention and interest in science) within the curriculum component of the conceptual framework was a particular strength $(M=4.83, S D=0.90)$ of the cooperating teachers in your school district. How does that match (or not) with what you would consider the strengths of cooperating teachers in your district to be, with respect to their preparedness to support science teacher candidates around curriculum?

\section{Quantitative Findings (Needs)}

The survey results indicated that the following construct of (1.5. design science curriculum that includes students obtaining, evaluating, and communicating information about systems of power, privilege, and marginalization) within the curriculum component of the conceptual framework was a particular need $(M=3.75, S D=1.42)$ of the cooperating teachers in your school district. How does that match (or not) with what you would consider the needs of cooperating teachers in your district to be with respect to their preparedness to support science teacher candidates around curriculum? 


\section{Qualitative Findings}

The following themes related to both curriculum and instruction were identified from the open-ended prompt, "Please describe your thoughts about the ratings you selected within the curriculum section." Cooperating teachers described that when it comes to supporting teacher candidates to develop culturally sustaining curriculum they tend to use resources acquired through in-service professional development or professional conferences. What comes to your mind as you think about these themes for curriculum within your own experience supporting science teacher candidates to meet the needs of diverse learners in your district?

\section{Instruction}

Quantitative Findings (Strengths)

The survey results indicated that the following construct (2.3. create a community of learners by encouraging students to focus on collective work, responsibility, and cooperation when learning science) within the instruction component of the conceptual framework was a particular strength $(M=4.75, S D=1.01)$ of the cooperating teachers in your school district. How does that match (or not) with what you would consider the strengths of cooperating teachers in your district to be with respect to their preparedness to support science teacher candidates around instruction?

\section{Quantitative Findings (Needs)}

The survey results indicated that the following construct (2.2. use a variety of linguistic styles with culturally diverse students in an attempt to communicate in culturally responsive or sustaining ways during science instruction) within the instruction component of the conceptual framework was a particular need $(M=3.67, S D=1.37])$ of the cooperating teachers in your school district. How does that match (or not) with what

you would consider the needs of cooperating teachers in your district to be with respect to their preparedness to support science teacher candidates around instruction?

\section{Qualitative Findings}

The qualitative themes identified for instruction from the open-ended prompt, "Please describe your thoughts about the ratings you selected within the instruction section." were very similar to those qualitative themes identified for the curriculum section. Cooperating teachers described that when it comes to supporting teacher candidates to facilitate student-centered learning they tend to use strategies that they learned during in-service professional development or professional conferences. What comes to your mind as you think about these themes for instruction within your own experience supporting science teacher candidates to meet the needs of diverse learners in your district?

\section{Relationships}

Quantitative Findings (Strengths)

The survey results indicated that essentially all of the constructs from the relationship component of the conceptual framework were a particular strength $(M=4.5$ or greater for 
all constructs, $S D=0.95-1.38$ ) of the cooperating teachers in your school district. How does that match (or not) with what you would consider the strengths of cooperating teachers in your district to be with respect to their preparedness to support science teacher candidates to develop relationships of dignity and care with their students?

\section{Qualitative Findings}

When it came to the relationships component of the conceptual framework, qualitative comments were made about both strategies to support science teacher candidates to develop relationships with their students as well as the importance of this aspect of a science teachers practice. What comes to your mind as you think about these two areas of qualitative comments within your own experience supporting science teacher candidates to meet the needs of diverse learners in your district?

\section{Synthesis/Closing}

Based on your experience as a cooperating teacher in your district and these preliminary survey results, how would you describe the strengths for cooperating teachers in your district to support science teacher candidates to use pedagogies that are culturally sustaining for traditionally underserved learners? Is there anything else you want to say about the preparedness of cooperating teachers in your district to support the science teacher candidates placed in their classrooms? 